\title{
A FUNCTIONAL INTEGRAL APPROACHES TO THE MAKEENKO-MIGDAL EQUATIONS
}

\author{
BRUCE K. DRIVER
}

\begin{abstract}
Makeenko and Migdal (1979) gave heuristic identities involving the expectation of the product of two Wilson loop functionals associated to splitting a single loop at a self-intersection point. Kazakov and I. K. Kostov (1980) reformulated the Makeenko-Migdal equations in the plane case into a form which made rigorous sense. Nevertheless, the first rigorous proof of these equations (and their generalizations) was not given until the fundamental paper of T. Lévy (2011). Subsequently Driver, Kemp, and Hall (2017) gave a simplified proof of Lévy's result and then with F. Gabriel (2017) we showed that these simplified proofs extend to the Yang-Mills measure over arbitrary compact surfaces. All of the proofs to date are elementary but tricky exercises in finite dimensional integration by parts. The goal of this article is to give a rigorous functional integral proof of the Makeenko-Migdal equations guided by the original heuristic machinery invented by Makeenko and Migdal. Although this stochastic proof is technically more difficult, it is conceptually clearer and explains "why" the Makeenko-Migdal equations are true. It is hoped that this paper will also serve as an introduction to some of the problems involved in making sense of quantizing Yang-Mill's fields.
\end{abstract}

\section{Contents}

1. Introduction

1.1. Guide to the reader [5]

1.2. Acknowledgements 6

2. Stochastic formulation and statement of the theorems 6

2.1. The stochastic framework 6

2.2. Statement of the theorems

2.3. Proof of Theorem 2.21 16

3. Heuristic arguments 16

3.1. Heuristic integration by parts 16

3.2. Heuristic proof of Theorem 2.23

3.3. Path expansions $\quad 22$

3.4. Heuristic proof of Theorem 2.27 24

4. Rigorous integration by parts 25

4.1. Rotating and translating white noise 25

4.2. Perturbations of $f, M^{f}$, and $/ / f$

4.3. Proof of Theorem 2.23

5. Loop expansion of parallel translation 31

5.1. Proof of Theorem 2.27 38

Appendix A. Appendix: connections, parallel translation, and curvature 40

2010 Mathematics Subject Classification. $81 \mathrm{~T} 13$ (primary), 60H30, 81T40. 
A.1. Transformation properties 40

A.2. Differential properties of parallel translation 42

Appendix B. Homotopy gauge fixing of Yang-Mills 44

B.1. Group actions and gauges 44

B.2. Disintegration formulas

B.3. Abstract gauge fixing 47

B.4. Yang-Mills gauge fixing 51

B.5. Area preserving diffeomorphisms

References

January 15, 2019 File:mmrigsmall.tex

\section{INTRODUCTION}

Let $K$ be any compact Lie group with $\mathfrak{k}=$ Lie $(K)$ being its Lie algebra which is assumed to be equipped with an $\operatorname{Ad}_{K}$ - invariant inner product denoted by $\langle\cdot, \cdot\rangle_{\mathfrak{k}}$ or often by $\langle\cdot, \cdot\rangle$. [For notational simplicity (and without loss of generality) we will assume that $K$ is a closed matrix Lie-subgroup of $\mathbb{C}^{D \times D}$ - the space of $D \times D$ complex matrices for some $D \in \mathbb{N}$.] Further suppose that $(M, g, o)$ is a pointed $d$-dimensional Riemannian manifold and $\mathrm{Vol}_{g}$ is the Riemannian volume measure on $M$. [We will soon specialize to the case where $d=2, M=\mathbb{R}^{2}, o=0$, and $g$ is the usual Euclidean metric in which case $\operatorname{Vol}_{g}$ is Lebesgue measure $(m)$ on $\mathbb{R}^{2}$.] Throughout the paper we write $\dot{k}(t)$ for $d k(t) / d t$ and $h^{\prime}(s)$ for $d h(s) / d s$, i.e. upper-dot and prime stand for $t$ and $s$-derivatives respectively.

Notation 1.1. Let $\mathcal{A}:=\Omega^{1}(M, \mathfrak{k})$ be the space of $\mathfrak{k}$ - valued connection one-forms on $M, \mathcal{G}$ be the gauge group consisting of functions $g: M \rightarrow K$ and $\mathcal{G}_{o}$ be the restricted gauge group defined by $\mathcal{G}_{o}=\{g \in \mathcal{G}: g(o)=I\}$. The smooth gauge group, $\mathcal{G}$, acts (as a right action) on $\mathcal{A}$ via,

$$
g \rightarrow A^{g}:=g^{-1} A g+g^{-1} d g \text { for all } g \in \mathcal{G} .
$$

where (locally) $A=\sum_{i=1}^{d} A_{i} d x_{i}$ with $A_{i}$ being locally defined $\mathfrak{k}$-valued functions on $M$.

Definition 1.2 (Covariant differentiation and parallel translation). Let $A \in \mathcal{A}$ be a k-valued connection one form on manifold $M$ and for a curve $\ell:[a, b] \rightarrow M$ which is absolutely continuous and a differentiable function $k:[a, b] \rightarrow K$, let $\nabla k(t)$ denote the covariant differential of $k$ defined by,

$$
\nabla_{t}^{A} k(t):=\frac{d}{d t} k(t)+A(\dot{\ell}(t)) k(t) .
$$

Also let $/ /_{t}^{A}(\ell) \in K$ be parallel translation along $\ell$ defined as the solution to the ODE (or more precisely its related integral equation),

$$
\nabla_{t}^{A} / /_{t}^{A}(\ell)=\frac{d}{d t} / /_{t}^{A}(\ell)+A(\dot{\ell}(t)) / /_{t}^{A}(\ell)=0 \text { with } / /{ }_{a}^{A}(\ell)=I \in K .
$$

We typically write $/ /{ }^{A}(\ell)$ for $/ /{ }_{b}^{A}(\ell)$. 
Definition 1.3 (Curvature). The curvature two form of $A \in \mathcal{A}$ is $F^{A}=d A+A \wedge A \in$ $\Omega^{2}(M, \mathfrak{k})$, i.e. for all $p \in M$ and $v, w \in T_{p} M$,

$$
F^{A}(v, w)=d A(v, w)+[A(v), A(w)]_{\mathfrak{k}} .
$$

See Theorem A.1 of Appendix A to see how the gauge group acts on $/ /{ }_{t}^{A}(\ell)$ and $F^{A}$.

Definition 1.4 (Yang-Mills Energy). The Yang-Mills energy associated to $A \in \mathcal{A}$ is defined by,

$$
\left\|F^{A}\right\|^{2}=\int_{M}\left|F^{A}\right|^{2}(x) d \operatorname{Vol}_{g}(x)
$$

where, for $p \in M$ and any orthonormal basis, $\left\{e_{i}\right\}_{i=1}^{d}$, of $T_{p} M$,

$$
\left|F^{A}\right|^{2}(p)=\sum_{1 \leq i<j \leq d}\left|F^{A}\left\langle e_{i}, e_{j}\right\rangle\right|_{\mathfrak{k}}^{2} .
$$

Now suppose that $M=\mathbb{R}^{d}$ for some $d \in \mathbb{N}$ and let $m$ denote Lebesgue measure on $\mathbb{R}^{d}$. For $u, v \in L^{2}\left(\mathbb{R}^{d}, m ; \mathfrak{k}\right)$, let

$$
\begin{aligned}
\langle u, v\rangle & :=\int_{\mathbb{R}^{d}}\langle u(\cdot), v(\cdot)\rangle_{\mathfrak{k}} d m \text { and } \\
\|u\|^{2} & :=\langle u, u\rangle=\int_{\mathbb{R}^{d}}|u|_{\mathfrak{k}}^{2} d m .
\end{aligned}
$$

The informal Euclidean Yang-Mills' "measure" on $\mathcal{A}$ is the expression

$$
d \mu_{\mathrm{YM}_{d}}(A)=\frac{1}{Z} \exp \left(-\frac{1}{2}\left\|F^{A}\right\|^{2}\right) \mathcal{D} A
$$

where $\mathcal{D} A$ is a (non-existent) Lebesgue measure on $\mathcal{A}$ and $Z$ is a "normalizing constant." Since $F^{A^{g}}=\operatorname{Ad}_{g^{-1}} F^{A}$ (see Theorem A.1 below) and the $\langle\cdot, \cdot\rangle_{\mathfrak{k}}$ is an $\operatorname{Ad}_{K^{-}}$invariant inner product, the functional, $A \rightarrow\left\|F^{A}\right\|^{2}$, is invariant under the right action of $\mathcal{G}$ on $\mathcal{A}$ given in Eq. 1.1). As $\mathcal{G}$ is an infinite dimensional "infinite volume" group, it is not possible, even at this informal level, to interpret the expression on the right side of Eq. (1.4) as a probability measure on $\mathcal{A}$. As is customary, one should try to interpret $\mu_{\mathrm{YM}}$ as a measure on the quotient space $\mathcal{A} / \mathcal{G}_{0}$. To be more concrete one usually tries to "define" $d \mu_{\mathrm{YM}}$ by appropriately restricting $A$ in Eq. (1.4) to be in a slice, $\mathcal{A}_{0} \subset \mathcal{A}$, of the gauge group action. We will discuss "gauge fixing" in more detail in Appendix B below.

For the purposes of this paper we now specialize to $d=2$ so that $M=\mathbb{R}^{2}$ equipped with its standard coordinates, $(x, y)$. We will further take $\mathcal{A}_{0}$ to be the connection one forms in the so called "complete axial" gauge.

Notation 1.5 (Complete axial gauge). Let $\mathcal{A}_{0}$ denote the subspace functions $A: \mathbb{R}^{2} \rightarrow \mathfrak{k}$ such that $A(x, 0)=0$ for all $x \in \mathbb{R}$. We will identify $A \in \mathcal{A}_{0}$ with the connection one form $A d x$ and refer to $A \in \mathcal{A}_{0}$ as a connection one form in the complete axial gauge.

If $A: \mathbb{R}^{2} \rightarrow \mathfrak{k}(A(x, 0)$ need not be zero yet), then the curvature 2-form $A d x$ is given simply by $F^{A d x}=f^{A} d x \wedge d y$ where $f^{A}:=-\partial_{y} A$ is the curvature function associated to $A$. Conversely 
given a (continuous say) function, $f: \mathbb{R}^{2} \rightarrow \mathfrak{k}$, we may define $A \in A_{0}$ by

$$
A(x, y)=-\left[\int_{0}^{y} f\left(x, y^{\prime}\right) d y^{\prime}\right] .
$$

Thus Eq. (1.5) sets up a linear isomorphism between $\mathfrak{k}$-valued curvature functions $(f)$ on $\mathbb{R}^{2}$ and elements of $\mathcal{A}_{0}$ with the inverse operation given by $A \rightarrow f=-\partial_{y} A$.

Restricting the expression in Eq. (1.4) to $\mathcal{A}_{0}$ leads us to consider the informally defined "measure,"

$$
d \mu(A)=“ \frac{1}{Z} \exp \left(-\frac{1}{2}\left\|-\partial_{y} A\right\|^{2}\right) \mathcal{D} A, "
$$

where again $\mathcal{D} A$ represents an ill defined Lebesgue measure on $\mathcal{A}_{0}$ and $Z$ is chosen to make $\mu$ a probability measure. [Justification for using Eq. (1.6) is provided in Appendix B below.] With this notation and formally using standard finite dimensional Gaussian integral formulas in this infinite dimensional setting, we should have

$$
\mathbb{E}\left[e^{i\langle f, u\rangle}\right]=\frac{1}{Z} \int_{\mathcal{A}_{0}} e^{i\left\langle-\partial_{y} A, u\right\rangle} \exp \left(-\frac{1}{2}\left\|\partial_{y} A\right\|^{2}\right) \mathcal{D} A=e^{-\frac{1}{2}\|u\|^{2}},
$$

where, as usual, we use "E"E" to denote integration relative to a probability measure. In other words, for all $u \in L^{2}\left(\mathbb{R}^{2}, m ; \mathfrak{k}\right)$, we should interpret $\langle f, u\rangle$ to be a mean zero Gaussian random variable with variance equal to $\|u\|^{2}$.

Starting with either Eqs. 11.6 or Eq. 11.7) along with Eq. 11.5), we ultimately want to understand expectations of gauge invariant functions, $\Psi: \mathcal{A} \rightarrow \mathbb{C}$. The typical example of such functions are the so called Wilson functionals, i.e. functions on $\mathcal{A}$ of the form $\Psi(A):=$ $U\left(\left\{/ /^{A}\left(\sigma_{i}\right)\right\}_{i=1}^{N}\right)$ where $U: K^{N} \rightarrow \mathbb{C}$ and $\left\{\sigma_{i}\right\}_{i=1}^{N}$ is a collection of paths in $\mathbb{R}^{2}$ all chosen so that $\Psi$ is gauge invariant. The prototypical examples of Wilson functionals is to take $\Psi$ to be a function of Wilson loop variables, i.e. $\Psi(A):=U\left(\left\{\operatorname{tr}\left[/ /{ }^{A}\left(\sigma_{i}\right)\right]\right\}_{i=1}^{N}\right)$ where "tr" is the matrix trace and each $\sigma_{i}$ is now assumed be a loop in the plane. The main goal of this paper is to give a stochastic proof Makeenko-Migdal identities, see Theorems 2.23 and Theorems 2.21 below. At the heart of these equations is an infinite dimensional integration by parts argument with gaugefield theory complications. [Without the complications of gauge invariance, the Makeenko-Migdal identities would likely be referred to as Schwinger-Dyson equations in the quantum filed theory litterature.] Although most interacting field theories are still not on firm mathematical grounds, formal integration in heuristic path integral expressions gives significant insight and constraints on the underlying quantum field theory.

For the $M=\mathbb{R}^{2}$ - setting with $K=U(N)$, in the fundamental paper [24] (which appeared on the archive in 2011), T. Lévy was able to show in the $N \rightarrow \infty$ limit that that the Wilson loop functionals can, with a little added information, be completely recovered from the MakeenkoMigdal identities. Recent significant progress in this direction when $M$ is a sphere or even general compact surface may be found in [8] and [20] respectively. The reader is also referred to these papers and to [24] and [1] for more background on (generalized) gauge fields over two dimensional manifolds.

The original Makeenko and Migdal (heuristic) identities, in any dimension, were the subject of [25]. V. A. Kazakov and I. K. Kostov (1980) in [22, Section 4] showed in the plane case that one side of the MM identity may be interpreted as the alternating sum of derivatives of the Wilson 
loop functional with respect to the areas of the faces surrounding a simple crossing, see also 23 , Equation 9] and Gopakumar and Gross (1995) [16, Equation 6.4]. T. Lévy [24] (appeared in 2011) was the first to provide a rigorous proof of the planar Makeenko-Migdal equations and also introduced a more general form of the equations. Lévy's proof of the generalized Makeenko-Migdal equation (gMM for short) are finite dimensional in nature and are based on Wilson functional expectation formulas developed in [13] and also [19].

A different proof of Lévy's gMM equations was subsequently given by A. Dahlqvist (2014) in [9]. Recently, in [15], three new proofs of the gMM identity were given and then later in [14] it was shown two of these proofs also work in the context of the Yang-Mills measure over an arbitrary compact surface. The latter extension replaces the planar formula from [13] by their extensions to compact surfaces developed by A. Sengupta [30, 31, 32, 33].

Up to now, all of the rigorous proofs of the gMM equations have been elementary but tricky exercises in finite dimensional integration by parts. The main aims of this paper are; 1) to explain the original heuristic infinite dimensional integration by parts arguments of the Makeenko-Migdal equations in more detail and precision, and 2) to then make (using stochastic calculus) these heuristic arguments rigorous.

One would certainly like to develop the theory here in the physically interesting case of $d=4$. However, it is still unknown how to make sense out of Eq. (1.4) when $d \geq 3$. (The $d=4$ case is one aspect of the Clay-Mathematics Millennium problem dealing with quantized YangMills fields.) There is however some very promising recent progress in this direction. Regarding the lattice approximations to $\mu_{\mathrm{YM}_{d}}$, see Chatterjee [5 [, [ [6], and especially [7] where the lattice normalization constant is shown to be under "control" as the lattice spacing tends to zero. On another front, the reader is directed to the work of Charalambous and L. Gross [2, 3, 4] and L. Gross [17] where the general theme is to develop the Yang-Mills heat equation as a method of regularizing the Wilson loop variables when $d \geq 3$. An outline of this strategy (which coupled with [7] may finally lead to a construction of quantized Yang-Mills fields when $d=4$ ) is given in the introduction in [3].

We finish this introduction with a road map of this paper. The author would also like to empahsize that the this paper is a rigorous interpretation of the extremely illuminating heuristic discussion of the MM equations given in the introduction of [24].

1.1. Guide to the reader. In section 2 we will formally introduce the $\mathrm{YM}_{2}$-expectations and give the statements of the main theorems listed here.

(1) Theorem 2.14 reviews the structure of the $\mathrm{YM}_{2}$-expectations as worked out in [13]. [For a perturbative theory perspective of these expectations the reader is directed to T. Nguyen's very interesting papers [26, 28, 27, 29].]

(2) Theorem 2.21 is a statement of T. Lévy's generalized form of the Makeenko-Migdal equations, see [24].

\footnotetext{
${ }^{1}$ In Theorem 7.1 of [5], Chatterjee introduces a "generalized Schwinger-Dyson equation for $S O(N)$ which in fact is a non-intrinsic writing of the standard Green's identity for $S O(N)$, i.e. integration by parts for the Laplacian on $S O(N)$. He is able to make good use of this non-intrinsic formula to find interesting convergent series expansions for loop expectations in the lattice model. However, the integration by parts used by Chatterjee seems to not be very closely related to what Makeenko and Migdal did in [25].
} 
(3) Theorem 2.23 is a white noise integration by parts formula relating one side of the gMM equation to an expression directly involving the curvature white noise function. This is the first main theorem of the paper.

(4) Theorem 2.27 is a Wilson-loop expansion formula which relates curvature white noise expression in Theorem 2.23 to the other side of the gMM equations.

(5) The proof of Theorem 2.21 is a simple consequence of Theorems 2.23 and 2.27 as explained at the end of Section 2 ,

In Section 3 we will give heuristic "proofs" of Theorems 2.23 and 2.27. The rigorous proofs of Theorems 2.23 and 2.27 will be given in Sections 4 and Section 5 respectively. Lastly there are two appendices to this paper. Appendix A reviews a few basic facts involving curvature and parallel translation. Appendix B reviews "homotopy" gauges and their relevance for interpreting the informal Yang-Mills' expectations which are suggestively described by Eq. (1.4.

1.2. Acknowledgements. It is a pleasure to acknowledge useful discussions with Brian Hall, Len Gross, and Todd Kemp pertaining to this work.

\section{Stochastic Formulation AND STATEMEnt of the theOREMS}

2.1. The stochastic framework. The heuristics Eq. (1.7), suggests that the random curvature function, " $f=-\partial_{y} A$ ", should be taken to be a $\mathfrak{k}$-valued white noise as in the next definition.

Definition 2.1 (White noise). A $\mathfrak{k}$-valued white noise is a probability space, $(\Omega, \mathcal{B}, \mathbb{P})$, along with a linear map, $f: L^{2}\left(\mathbb{R}^{2}, m ; \mathfrak{k}\right) \rightarrow L^{2}(\Omega, \mathbb{P} ; \mathbb{R})$, such that $f(u)$ is a mean zero Gaussian random variable with covariance $\mathbb{E}\left[[f(u)]^{2}\right]=\|u\|_{2}^{2}$ for each $u \in L^{2}\left(\mathbb{R}^{2}, m ; \mathfrak{k}\right)$.

We will typically write $f(u)$ as $\langle f, u\rangle$ and informally think that $\langle f, u\rangle$ is given as in Eq. 1.2 even though $f(x, y)$ has no meaning as a random variable for any $(x, y) \in \mathbb{R}^{2}$.

Notation 2.2. If $(\Omega, \mathcal{B}, \mathbb{P}, f)$ is a $\mathfrak{k}$-valued white noise and $B$ is a finite area Borel subset of $\mathbb{R}^{2}$, we abuse notation and let $f(B)$ (informally thought of as $\left.\int_{B} f(x, y) d x d y\right)$ ) be the random variable (well defined a $\mathbb{P}$-a.e.) which satisfies,

$$
\langle f(B), \xi\rangle_{\mathfrak{k}}:=\left\langle f, 1_{B} \xi\right\rangle \text { for all } \xi \in \mathfrak{k}
$$

A version of $f(B) \in L^{2}(\Omega, \mathbb{P} ; \mathfrak{k})$ is given by $f(B)=\sum_{\xi \in \beta}\left\langle f, 1_{B} \xi\right\rangle \xi$ where $\beta \subset \mathfrak{k}$ is any orthonormal basis for $\mathfrak{k}$. From the white noise, $f$, we may formally use Eq. 1.5 to recover the random (distribution valued) connection one form, A. Let us recall L. Gross's method (as explained in [13, 19]) on how to rigorously do this and then how to use stochastic calculus to interpret the resulting random parallel translation operators associated to "tame" curves in the plane.

Definition 2.3 (Horizontal curves). A horizontal curve is a path, $\ell:[a, b] \rightarrow \mathbb{R}^{2}$, of the form $\ell(t)=(t, y(t))$ where $y:[a, b] \rightarrow \mathbb{R}$ is a continuous function on a compact interval, $[a, b]$. Further let $R^{\ell}(t)$ be the region between the curve $y$ and the $x$-axis over interval $[a, t]$, see Figure 1 .

Again working formally, for $A d x \in \mathcal{A}_{0}$ we integrate $f=-\partial_{y} A$ to find

$$
A(x, y)=-\int_{0}^{y} f\left(x, y^{\prime}\right) d y^{\prime}
$$




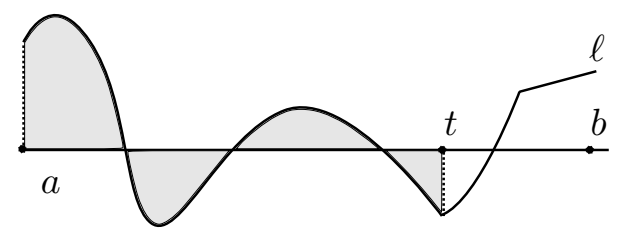

Figure 1. A typical horizontal curve with $R^{\ell}(t)$ being the shaded region.

and so if $\ell(t)=(t, y(t))$ is a horizontal curve as in Definition 2.3, then

$$
A d x\langle\dot{\ell}(\tau)\rangle=A(\tau, y(\tau))=-\int_{0}^{y(\tau)} f\left(\tau, y^{\prime}\right) d y^{\prime} .
$$

We now integrate this expression to define,

$$
\begin{aligned}
M_{t}^{f}(\ell) & :=\int_{a}^{t} A d x\langle\dot{\ell}(\tau)\rangle d \tau=-\int_{a}^{t}\left[\int_{0}^{y(\tau)} f\left(\tau, y^{\prime}\right) d y^{\prime}\right] d \tau \\
& =-\int_{R^{\ell}(t)} \hat{f}(x, y) d x d y,
\end{aligned}
$$

where, for a function $u: \mathbb{R}^{2} \rightarrow \mathfrak{k}$, we let

$$
\hat{u}(x, y):=\operatorname{sgn}(y) u(x, y)=\left\{\begin{array}{cll}
u(x, y) & \text { if } \quad y \geq 0 \\
-u(x, y) & \text { if } \quad y \leq 0
\end{array} .\right.
$$

By the construction of $M_{t}^{f}(\ell)$ in Eq. $2.1 p, \dot{M}_{t}^{f}(\ell)=A d x\langle\dot{\ell}(t)\rangle$ and so (writing $/ /{ }_{t}^{A}(\ell)$ for $\left./ /{ }_{t}^{A d x}(\ell)\right)$, we should have

$$
\frac{d}{d t} / /_{t}^{A}(\ell)+\dot{M}_{t}^{f}(\ell) / /_{t}^{A}(\ell)=0 \text { with } / /_{a}^{A}(\ell)=I \in K .
$$

In the stochastic setting $f$ and $A$ are distribution valued random variables and hence Eqs. (2.2) and (2.4) will require proper interpretation which is provided in Definitions 2.6 and 2.7 below. Before these key definition we need a little more preparation.

Definition 2.4. If $f$ is a white noise define $\hat{f}$ to be the white noise determined by

$$
\langle\hat{f}, u\rangle=\langle f, \hat{u}\rangle \text { for all } u \in L^{2}\left(\mathbb{R}^{2}, m ; \mathfrak{k}\right) \text {. }
$$

Let $\mathcal{B}_{\mathbb{R}^{2}}^{0}$ denote the finite area Borel subsets of $\mathbb{R}^{2}$. Notice that if $B \in \mathcal{B}_{\mathbb{R}^{2}}^{0}$, then

$$
\hat{f}(B)=\left\langle f, \widehat{1_{B}}\right\rangle=f\left(B \cap H_{+}\right)-f\left(B \cap H_{-}\right)
$$

where $H_{+}$and $H_{-}$denote the upper and lower half planes in $\mathbb{R}^{2}$.

Definition 2.5 (Filtration). For $s \in \mathbb{R}$, let

$$
\mathcal{F}_{s}^{0}=\sigma\left(f(B): B \in \mathcal{B}_{\mathbb{R}^{2}}^{0} \text { s.t. } B \subset(-\infty, s) \times \mathbb{R}\right)
$$

and then let $\left\{\mathcal{F}_{s}\right\}_{s \in \mathbb{R}}$ be the filtration on $(\Omega, \mathcal{F}, \mathbb{P})$ which is the right continuous version of $\left\{\mathcal{F}_{s}^{0}\right\}_{s \in \mathbb{R}}$ augmented by the zero sets of $\mathcal{F}$. 
Defining $M_{t}^{f}(\ell):=-\hat{f}\left(R^{\ell}(t)\right)$ as indicated in Eq. 2.2 implies that $\left\{M_{t}^{f}(\ell): a \leq t \leq b\right\}$ is a mean zero $\mathfrak{k}$-valued Gaussian process with

$$
\mathbb{E}\left[\left\langle M_{t}^{f}(\ell), \xi_{1}\right\rangle\left\langle M_{\tau}^{f}(\ell), \xi_{2}\right\rangle\right]=\left\langle\xi_{1}, \xi_{2}\right\rangle_{\mathfrak{k}} m^{2}\left(R^{\ell}(t \wedge \tau)\right) \forall \xi_{1, \xi_{2}} \in \mathfrak{k} \text { and } a \leq t, \tau \leq b .
$$

This process has independent increments and is a time change of $\mathfrak{k}$-valued Brownian motion and hence has a continuous version. The existence of a continuous version also follows using Kolmogorov's continuity criteria along with the observation that (for $t>\tau$ )

$$
\mathbb{E}\left[\left|M_{t}^{f}(\ell)-M_{\tau}^{f}(\ell)\right|_{\mathfrak{k}}^{2}\right]=\operatorname{dim} K \cdot m\left(R^{\ell}(t) \backslash R^{\ell}(\tau)\right)=\operatorname{dim} K \cdot \int_{\tau}^{t}|y(x)| d x \leq C|t-\tau|
$$

which, because $\left\{M_{t}^{f}(\ell)\right\}_{a \leq t \leq b}$ is a Gaussian process, implies for all $p \geq 2$, there exists $C_{p}<\infty$ such that

$$
\mathbb{E}\left[\left|M_{t}^{f}(\ell)-M_{\tau}^{f}(\ell)\right|_{\mathfrak{k}}^{p}\right] \leq C_{p}|t-\tau|^{p / 2} .
$$

Definition 2.6 (Martingales). From now on we assume that $M_{t}^{f}(\ell)$ refers to a continuous version of $[a, b] \ni t \rightarrow-\hat{f}\left(R^{\ell}(t)\right)$. This version becomes a continuous $\mathfrak{k}$-valued martingale adapted to the filtration $\left\{\mathcal{F}_{s}\right\}_{s \in \mathbb{R}}$.

Motivated by Eq. (2.4) we now defined the stochastic parallel translation as follows.

Definition 2.7 (Stochastic Parallel Translation). If $[a, b] \ni t \rightarrow \ell(t)=(t, y(t)) \in \mathbb{R}^{2}$ is $a$ horizontal curve as in Definition 2.3, le ${ }^{2} / /{ }_{t}^{f}(\ell)$ be the $K$-valued random process which is defined as the solution to the stochastic differential equation;

$$
d / /_{t}^{f}(\ell)+\delta M_{t}^{f}(\ell) /{ }_{t}^{f}(\ell)=0 \text { with } / / a(\ell)=I \in K,
$$

where $\delta M_{t}^{f}(\ell)$ denotes the Fisk-Stratonovich differential of $M^{\ell}$. Parallel translation along any purely vertical path in $\mathbb{R}^{2}$ is defined to be the constant function $I \in K$. We further simply write $/ / f(\ell)$ for $/ /{ }_{b}^{f}(\ell)$.

A directed path $\sigma$ in $\mathbb{R}^{2}$ is tame if it is the concatenation of finitely many vertical paths and forwards and backwards horizontal paths. For a tame path $\sigma$ we define $/ / f(\sigma)$ in the natural way as the products of forward parallel translations for the forward paths and inverses of parallel translations for the backward horizontal paths. For example if $\sigma$ is the tame path shown in Figure 2 we let $/ /^{f}(\sigma)=/ /^{f}\left(\ell_{3}\right) / /^{f}\left(\ell_{2}\right)^{-1} / / f\left(\ell_{1}\right)$ where $\ell_{i}(t)=\left(t, y_{i}(t)\right)$ and $y_{1}:[a, c] \rightarrow \mathbb{R}$, $y_{2}:[b, c] \rightarrow \mathbb{R}$, and $y_{3}:[b, d] \rightarrow \mathbb{R}$ are the functions whose graphs are indicated in Figure 2 .

Notation 2.8. Given a directed tame path $\sigma$ in $\mathbb{R}^{2}$, let $\tilde{\sigma} \subset \mathbb{R}^{2}$ denote the image of $\sigma$ in $\mathbb{R}^{2}, \sigma_{f}$ denote the final point of $\sigma$ and $\sigma_{i}$ denote the initial point of $\sigma$, see Figure 2 . A tame graph, $\mathbb{G}$, in $\mathbb{R}^{2}$ is a finite collection of directed tame paths such that; if $\sigma, \tau$ are any two distinct elements in $\mathbb{G}$ then $\tilde{\sigma} \cap \tilde{\tau} \subset\left\{\sigma_{i}, \tau_{i}, \sigma_{f}, \tau_{f}\right\}$, see for example Figure 3. Let $V(\mathbb{G})=\cup_{\sigma \in \mathbb{G}}\left\{\sigma_{i}, \sigma_{f}\right\}$ denote the vertices of $\mathbb{G}$.

\footnotetext{
${ }^{2}$ To emphasize that the white noise is the fundamental input we are now writing $/ /_{t}^{f}(\ell)$ in place of $/ / t_{t}^{A}(\ell)$. Although, in the heuristic proof section 3 below, we will briefly revert back to the old notation.
} 


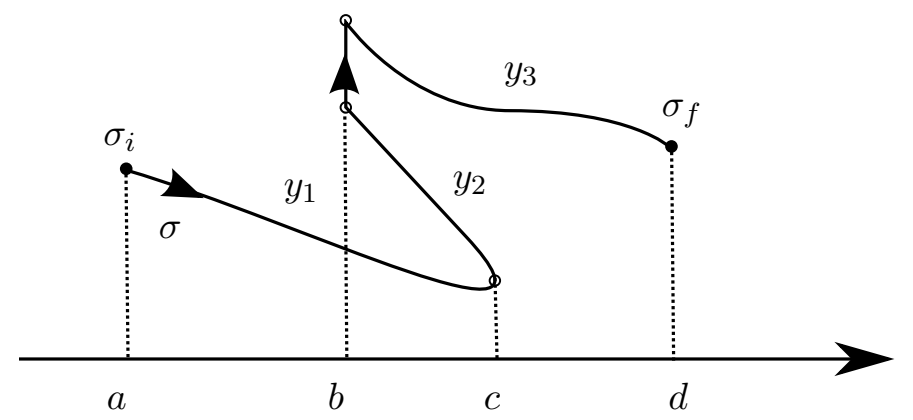

Figure 2. A tame path, $\sigma$, with one vertical segment, two forward horizontal segments, and one backwards horizontal segment.

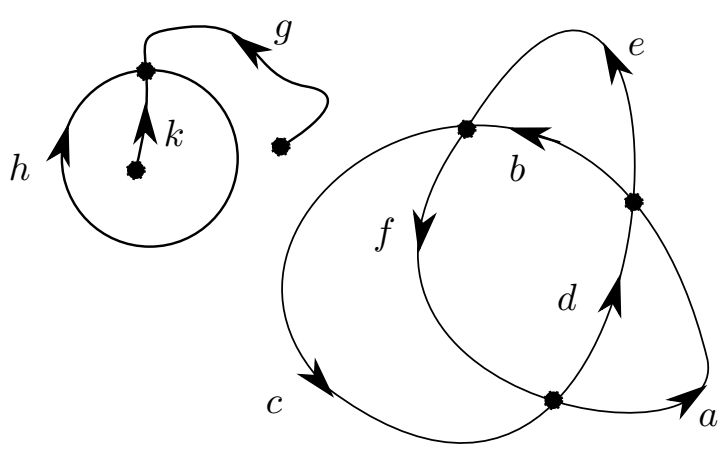

Figure 3. An example of a tame graph, $\mathbb{G}$. In this example $g_{f}=k_{f}=h_{f}=h_{i}$. Note, as in this graph, there is not assumption that tame graphs are connected.

Notation 2.9. To each directed tame graph, $\mathbb{G}$, in $\mathbb{R}^{2}$, let $K^{\mathbb{G}}$ be the the configuration space of all functions, $\omega: \mathbb{G} \rightarrow K$. Further let

$$
/ / f(\mathbb{G}):=\{/ / f(\sigma): \sigma \in \mathbb{G}\} \in K^{\mathbb{G}}
$$

be stochastic parallel translation along all the paths in $\mathbb{G}$. More accurately we should denote $/ / f(\mathbb{G})$ by $/ /\left.{ }^{f}\right|_{\mathbb{G}}$ but this notation is a bit cumbersome.

Definition 2.10 (Discrete gauge tranformations). A function $u: V(\mathbb{G}) \rightarrow K$ is called a discrete gauge transformation. To each such function $u: V(\mathbb{G}) \rightarrow K$ and $\omega \in K^{\mathbb{G}}$, we let $\omega^{u} \in K^{\mathbb{G}}$ be defined by

$$
\omega^{u}(\sigma)=u\left(\sigma_{f}\right)^{-1} \omega(\sigma) u\left(\sigma_{i}\right) \text { for all } \sigma \in \mathbb{G} .
$$

Example 2.11. If $x_{0} \in V(\mathbb{G}) \subset \mathbb{R}^{2}$ and $k \in K$ are given, then $u_{x_{0}, k}: V(\mathbb{G}) \rightarrow K$ defined by

$$
u_{x_{0}, k}(x)=\left\{\begin{array}{ccc}
k & \text { if } & x=x_{0} \\
I & \text { if } & x \neq x_{0}
\end{array}\right.
$$


is a discrete gauge transformation. Moreover any discrete gauge transformation may be written as a finite product of the $u_{x_{0}, k}$ via

$$
u=\prod_{x_{0} \in V(\mathbb{G})} u_{x_{0}, u\left(x_{0}\right)}
$$

where the above pointwise product is independent of any choice of ordering of the terms.

Definition 2.12 (Discrete gauge invariant functions). A function, $U: K^{\mathbb{G}} \rightarrow \mathbb{C}$, is said to be discrete gauge invariant at $x_{0} \in V(\mathbb{G})$ if $U\left(\omega^{u_{0}, k}\right)=U(\omega)$ for all $k \in K$ and $\omega \in K^{\mathbb{G}}$. The function, $U: K^{\mathbb{G}} \rightarrow \mathbb{C}$, is said to be discrete gauge invariant if $U\left(\omega^{u}\right)=U(\omega)$ for all $\omega \in K^{\mathbb{G}}$ and all discrete gauge transformations, $u: V(\mathbb{G}) \rightarrow K$. [Because of Eq. 2.6] it is easily seen that $U$ is discrete gauge invariant iff it is discrete gauge invariant at each $x_{0} \in V(\mathbb{G})$.]

Remark 2.13. For a tame graph, $\mathbb{G}$, in $\mathbb{R}^{2}$ and $A \in \mathcal{A}$, let (analogous to Notation 2.9)

$$
/ /^{A}(\mathbb{G}):=\left\{/ /^{A^{g}}(\sigma): \sigma \in \mathbb{G}\right\} \in K^{\mathbb{G}} .
$$

If $U: K^{\mathbb{G}} \rightarrow \mathbb{C}$ is a discrete gauge invariant function, then $\Psi: \mathcal{A} \rightarrow \mathbb{C}$ defined by $\Psi(A)=$ $U\left(/ /{ }^{A}(\mathbb{G})\right)$ is a gauge invariant function on $\mathcal{A}$. Indeed if $g \in C^{1}\left(\mathbb{R}^{2} \rightarrow K\right)$, then (see Eq. (A.2) of Appendix A)

$$
\begin{aligned}
\Psi\left(A^{g}\right) & =U\left(/ /^{A}(\mathbb{G})\right)=U\left(\left\{g\left(\sigma_{f}\right)^{-1} / /{ }^{A}(\sigma) g\left(\sigma_{i}\right)\right\}_{\sigma \in \mathbb{G}}\right) \\
& =U\left(/ /^{A}(\mathbb{G})\right)=\Psi(A) .
\end{aligned}
$$

When $U: K^{\mathbb{G}} \rightarrow \mathbb{C}$ is a discrete gauge invariant function, it is shown in $[13]$ that $\mathbb{E}[U(/ / f(\mathbb{G}))]$ may be computed as a finite dimensional integrals relative to a density constructed via certain products of the convolution heat kernel on $K$. The next theorem summarizes the information we need for the purposes of this paper.

Theorem 2.14 ([13]). If $\mathbb{G}$ is a directed tame graph and $U: K^{\mathbb{G}} \rightarrow \mathbb{C}$ is a bounded measurable discrete gauge invariant function, then

$$
\mathbb{E}[U(/ / f(\mathbb{G}))]=\int_{K^{\mathbb{G}}} U(\omega) \rho_{\mathbb{G}}(\omega) d \lambda_{\mathbb{G}}(\omega)
$$

where $\lambda_{\mathbb{G}}$ is normalized Haar measure on $K^{\mathbb{G}}$ and $\rho_{\mathbb{G}}$ is a smooth density function which is depends only on the topology of $\mathbb{G}$ and the areas $\left\{t_{i}\right\}_{i=1}^{N}$ of the bounded connected regions in $\mathbb{R}^{2} \backslash \mathbb{G}$. Moreover, relative to topological preserving perturbations of $\mathbb{G}, \mathbb{E}[U(/ / f(\mathbb{G}))]$ is a smooth function of $\left(t_{1}, \ldots, t_{N}\right)$.

The following corollary follows directly from Theorem 2.14. This corollary is also "explained" heuristically in Meta-Theorems B.55 and B.56 of Appendix B.

Corollary 2.15 (Area preserving diffeomorphism invariance). If $\varphi$ is any area preserving diffeomorphism of $\mathbb{R}^{2}$ such that $\varphi \circ \sigma$ is a tame path for all $\sigma \in \mathbb{G}$, then

$$
\mathbb{E}[U(/ / f(\mathbb{G}))]=\mathbb{E}\left[U\left(\{/ / f(\varphi \circ \sigma)\}_{\sigma \in \mathbb{G}}\right)\right] .
$$


Definition 2.16 ( $\sigma$-directional derivatives). For $\xi \in \mathfrak{k}, \sigma \in \mathbb{G}, \omega \in K^{\mathbb{G}}$, and $U: K^{\mathbb{G}} \rightarrow \mathbb{C}$ differentiable, let

$$
\left(\nabla_{\xi}^{\sigma} U\right)(\omega)=\left.\frac{d}{d t}\right|_{0} U\left(\left\{\omega(b) e^{t \delta_{\sigma, b} \xi}\right\}_{b \in \mathbb{G}}\right):=\left.\frac{d}{d t}\right|_{0} U\left(\omega_{t}\right)
$$

where, for $t \in \mathbb{R}$ and $b \in \mathbb{G}$,

$$
\omega_{t}(b)=\left\{\begin{array}{cc}
\omega(b) & \text { if } \quad b \neq \sigma \\
\omega(\sigma) e^{t \xi} & \text { if } \quad b=\sigma
\end{array} .\right.
$$

Lemma 2.17. If $\sigma \in \mathbb{G}, \xi \in \mathfrak{k}$, and $U: K^{\mathbb{G}} \rightarrow \mathbb{C}$ is discrete gauge invariant, then

$$
\left(\nabla_{\xi}^{\sigma} U\right)\left(\omega^{u}\right)=\left(\nabla_{\operatorname{Ad}_{u\left(\sigma_{i}\right)} \xi} U\right)(\omega) \forall \omega \in K^{\mathbb{G}} \text { and } u: V(\mathbb{G}) \rightarrow K .
$$

In particular if $u\left(\sigma_{i}\right)=I$, then $\left(\nabla_{\xi}^{\sigma} U\right)\left(\omega^{u}\right)=\left(\nabla_{\xi}^{\sigma} U\right)(\omega) \forall \omega \in K^{\mathbb{G}}$.

Proof. Since

$$
\begin{aligned}
& \omega^{u}(b) e^{t \delta_{\sigma, b} \xi}=u\left(b_{f}\right)^{-1} \omega(b) u\left(b_{i}\right) e^{t \delta_{\sigma, b} \xi} \\
& =u\left(b_{f}\right)^{-1} \omega(b) u\left(b_{i}\right) e^{t \delta_{\sigma, b} \xi} u\left(b_{i}\right)^{-1} u\left(b_{i}\right) \\
& =u\left(b_{f}\right)^{-1} \omega(b) e^{t \delta_{\sigma, b} \operatorname{Ad}_{u\left(b_{i}\right)} \xi} u\left(b_{i}\right)=u\left(b_{f}\right)^{-1} \omega(b) e^{t \delta_{\sigma, b} \operatorname{Ad}_{u\left(\sigma_{i}\right)} \xi} u\left(b_{i}\right),
\end{aligned}
$$

it follows using the discrete gauge invariance of $U$ that

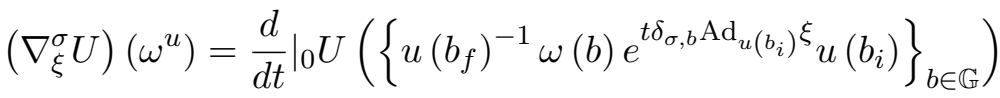

$$
\begin{aligned}
& =\left.\frac{d}{d t}\right|_{0} U\left(\left\{\omega(b) e^{t \delta_{\sigma, b} \operatorname{Ad}_{u\left(\sigma_{i}\right)} \xi}\right\}_{b \in \mathbb{G}}\right)=\left(\nabla_{\operatorname{Ad}_{u\left(\sigma_{i}\right)} \xi} U\right)(\omega) .
\end{aligned}
$$

One consequence of the previous lemma is that $\nabla_{\xi}^{\sigma} U$ is typically not discrete gauge invariant. In the next proposition, we will show that certain second order differential operators do preserve discrete gauge invariant functions.

Notation $2.18\left(\left(\nabla^{\sigma_{1}} \cdot \nabla^{\sigma_{2}} U\right)(\Gamma(f))\right)$. If $\sigma_{1}, \sigma_{2} \in \mathbb{G}$ and $U: K^{\mathbb{G}} \rightarrow \mathbb{C}$ is twice continuously differentiable, let

$$
\nabla^{\sigma_{1}} \cdot \nabla^{\sigma_{2}} U=\sum_{\xi \in \beta} \nabla_{\xi}^{\sigma_{1}} \nabla_{\xi}^{\sigma_{2}} U
$$

where $\beta$ is an orthonormal basis for $\mathfrak{k}$.

Since $\mathfrak{k} \times \mathfrak{k} \ni(\xi, \eta) \rightarrow\left(\nabla_{\eta}^{\sigma_{1}} \nabla_{\xi}^{\sigma_{2}} U\right)(\omega)$ is a bilinear form on $\mathfrak{k} \times \mathfrak{k}$, it is easily verified that the sum in Eq. (2.7) is independent of the choice of orthonormal basis, $\beta$, for $\mathfrak{k}$.

Proposition 2.19. Let $b, c$ be any two bonds in $\mathbb{G}$ such that $b_{i}=c_{i}=q$ as in Figure 4. Then if $U: K^{\mathbb{G}} \rightarrow \mathbb{C}$ is twice continuously differentiable and discrete gauge invariant then $\left(\nabla^{b} \cdot \nabla^{c} U\right)$ is still discrete gauge invariant. 


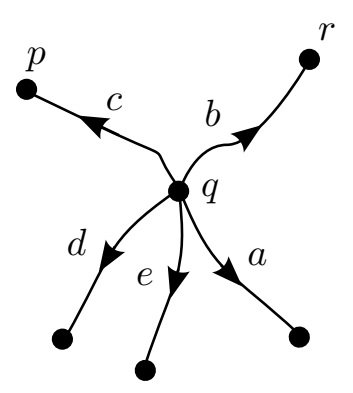

Figure 4. Two bonds among five bonds in $\mathbb{G}$ sharing $q$ as their initial points.

Proof. Let us first observe that

$$
\begin{aligned}
\left(\nabla_{\xi}^{b} \nabla_{\xi}^{c} U\right)(\omega) & =\left.\frac{d}{d s}\right|_{0}\left(\nabla_{\xi}^{c} U\right)\left(\left\{\omega(\sigma) e^{s \delta_{\sigma, b} \xi}: \sigma \in \mathbb{G}\right\}\right) \\
& =\left.\left.\frac{d}{d t}\right|_{0} \frac{d}{d s}\right|_{0}\left(\nabla_{\xi}^{c} U\right)\left(\left\{\omega(\sigma) e^{s \delta_{\sigma, b} \xi} e^{t \delta_{\sigma, c} \xi}: \sigma \in \mathbb{G}\right\}\right) \\
& =\left.\left.\frac{d}{d t}\right|_{0} \frac{d}{d s}\right|_{0} U\left(\left\{\omega(\sigma) e^{\left(s \delta_{\sigma, b}+t \delta_{\sigma, c}\right) \xi}: \sigma \in \mathbb{G}\right\}\right) .
\end{aligned}
$$

Thus if $u: V(\mathbb{G}) \rightarrow G$ is a discrete gauge transformation and $U$ is a discrete gauge invariant function, then

$$
\begin{aligned}
\left(\nabla_{\xi}^{b} \nabla_{\xi}^{c} U\right)\left(\omega^{u}\right) & =\left.\left.\frac{d}{d t}\right|_{0} \frac{d}{d s}\right|_{0} U\left(\left\{u\left(\sigma_{f}\right)^{-1} \omega(\sigma) u\left(\sigma_{i}\right) e^{\left(s \delta_{\sigma, b}+t \delta_{\sigma, c}\right) \xi}: \sigma \in \mathbb{G}\right\}\right) \\
& =\left.\left.\frac{d}{d t}\right|_{0} \frac{d}{d s}\right|_{0} U\left(\left\{u\left(\sigma_{f}\right)^{-1} \omega(\sigma) e^{\left(s \delta_{\sigma, b}+t \delta_{\sigma, c}\right) \operatorname{Ad}_{u\left(\sigma_{i}\right)} \xi} u\left(\sigma_{i}\right): \sigma \in \mathbb{G}\right\}\right) \\
& =\left.\left.\frac{d}{d t}\right|_{0} \frac{d}{d s}\right|_{0} U\left(\left\{\omega(\sigma) e^{\left(s \delta_{\sigma, b}+t \delta_{\sigma, c}\right) \operatorname{Ad}_{u(q)} \xi}: \sigma \in \mathbb{G}\right\}\right) \\
& =\left(\nabla_{\operatorname{Ad}_{u(q)} \xi}^{b} \nabla_{\operatorname{Ad}_{u(q)} \xi} U\right)(\omega) .
\end{aligned}
$$

Summing this equation on $\xi \in \beta$ using $\left\{\operatorname{Ad}_{u(q)} \xi\right\}_{\xi \in \beta}$ is still an orthonormal basis for $\mathfrak{k}$ shows $\left(\nabla^{b} \cdot \nabla^{c} U\right)\left(\omega^{u}\right)=\left(\nabla^{b} \cdot \nabla^{c} U\right)(\omega)$.

2.2. Statement of the theorems. We are now going to consider graphs, $\mathbb{G}$, in the plane that contain a simple crossing at some vertex $v \in \mathbb{R}^{2}$ as in Figure 5 below.

Definition 2.20. Let $\mathbb{G}$ be a directed graph in $\mathbb{R}^{2}$ with a simple crossing at $v \in V(\mathbb{G})$ as depicted in Figure 5. A function $U: K^{\mathbb{G}} \rightarrow \mathbb{C}$ is said to have extended gauge invariance at $v \in$ $V(\mathbb{G})$ if the edges $\sigma_{1}, \ldots, \sigma_{4}$ are distinct and the dependence of $U(\omega)$ on $\left\{\omega\left(\sigma_{i}\right)\right\}_{i=1}^{4}$ is through $\omega\left(\sigma_{1}\right) \omega\left(\sigma_{3}\right)^{-1}$ and $\omega\left(\sigma_{2}\right) \omega\left(\sigma_{4}\right)^{-1}$ only.

Extended gauge invariance at $v$ should be interpreted to mean that two otherwise independent paths happened to cross at $v \in \mathbb{R}^{2}$ and therefore both paths had to artificially be split at $v$ in order to make the given configuration of paths into a tame graph. An equivalent condition that $U: K^{\mathbb{G}} \rightarrow \mathbb{C}$ has extended gauge invariance at $v$ is to verify that

$$
K^{2} \ni(x, y) \rightarrow U\left(\omega\left(\sigma_{1}\right) x, \omega\left(\sigma_{4}\right) x, \omega\left(\sigma_{2}\right) y, \omega\left(\sigma_{4}\right) y,\left\{\omega(\sigma): \sigma \ni \mathbb{G} \backslash\left\{\sigma_{1}, \sigma_{2}, \sigma_{3}, \sigma_{4}\right\}\right\}\right)
$$




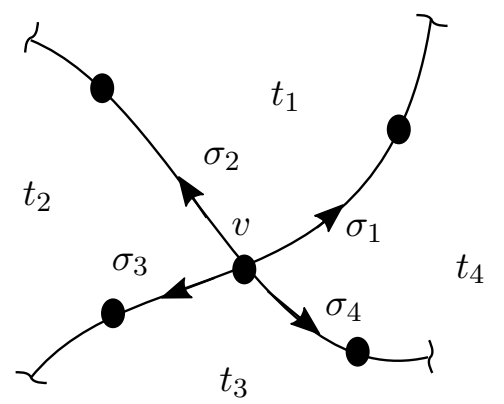

Figure 5. A simple crossing.

is a constant function for each $\omega \in K^{\mathbb{G}}$.

Theorem 2.21 (Extended M.M. Equations, Lévy 24]). Let $\mathbb{G}$ be a directed graph in $\mathbb{R}^{2}$ with a simple crossing at $v \in V(\mathbb{G})$ as depicted in Figure 5 and $U \in C^{2}\left(K^{\mathbb{G}} \rightarrow \mathbb{C}\right)$ be a gauge invariant function. If $U$ has extended gauge invariance at $v$, then

$$
\mathbb{E}\left[\left(\nabla^{\sigma_{1}} \cdot \nabla^{\sigma_{2}} U\right)\left(/ /^{f}(\mathbb{G})\right)\right]=-\left(\frac{\partial}{\partial t_{1}}-\frac{\partial}{\partial t_{2}}+\frac{\partial}{\partial t_{3}}-\frac{\partial}{\partial t_{4}}\right) \mathbb{E}\left[U\left(/ /^{f}(\mathbb{G})\right)\right]
$$

where $\left\{t_{i}\right\}_{i=1}^{4}$ refers to the areas described in Figure 5. IIf any one of these areas are infinite the corresponding derivative should be omitted from the formula.]

We begin with a simple reduction on the geometry of the crossing in Figure 5 .

Proposition 2.22. We may find an area preserving diffeomorphism, $\varphi: \mathbb{R}^{2} \rightarrow \mathbb{R}^{2}$, such that the simple crossing in Figure 5 may be transformed into the straight line crossing pattern (i.e. $e_{j}=\varphi \circ \sigma_{j}$ for $\left.1 \leq j \leq 4\right)$ going through $0 \in \mathbb{R}^{2}$ as in Figure 6 .

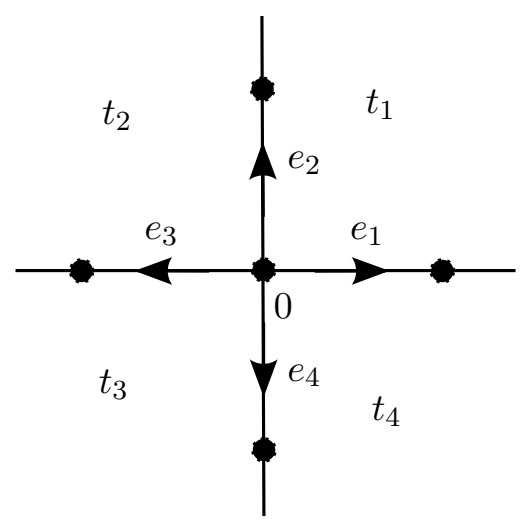

FiguRE 6 . A very simple basic configuration for $\mathbb{G}$ near $0 \in \mathbb{R}^{2}$.

Proof. First suppose that $h:[a, b] \rightarrow \mathbb{R}$ is a $C^{1}$-function, $[a, b] \ni t \rightarrow \sigma(t):=(t, h(t))$ is the associated horizontal curve, and $t_{0} \in(a, b)$ is a given point. Let $\theta \in C_{c}^{\infty}((a, b),[0,1])$ be chosen 
so that $\theta=1$ near $t_{0}$ and then define

$$
\begin{aligned}
\varphi(x, y) & :=\left(x-t_{0}, y-\theta(x) h(x)\right) \text { and } \\
\tilde{h}(s) & :=h\left(t_{0}+s\right)\left(1-\theta\left(t_{0}+s\right)\right) \text { for } s \in\left[a-t_{0}, b-t_{0}\right],
\end{aligned}
$$

where $\theta(x) h(x) \equiv 0$ for $x \notin[a, b]$. It is now a simple matter to verify;

(1) $\varphi$ is an area preserving diffeomorphism.

(2) If $t \in[a, b]$ and $s:=t-t_{0}$, then

$$
\varphi(t, h(t))=\left(t-t_{0}, h(t)-\theta(t) h(t)\right)=(s, \hat{h}(s)) .
$$

Thus $\varphi$ transforms the horizontal path $\sigma$ to the horizontal path $\tilde{\sigma}(s):=(s, \tilde{h}(s))$ which lies on the $x$-axis for $s$ near 0 .

(3) Moreover, $\varphi\left(t_{0}, y\right)=\left(0, y-h\left(t_{0}\right)\right)$ so that the vertical path going through $\sigma\left(t_{0}\right)=$ $\left(t_{0}, h\left(t_{0}\right)\right)$ is transformed into the vertical path going through $\tilde{\sigma}(0)=(0,0)$.

We now construct $\varphi$ as in the theorem as a composition of a number or area preserving diffeomorphisms as follows. Let $\sigma_{2} * \bar{\sigma}_{4}$ denote the path in $\mathbb{R}^{2}$ which follows $\sigma_{4}$ backwards and then continues to follow the path $\sigma_{2}$. Choose a rotation which rotates $\sigma_{2} * \bar{\sigma}_{4}$ into a path which is almost horizontal, then apply the above construction to find a $\varphi$ which makes the resulting curve lie on the $x$-axis, and then rotate this curve back to a vertical curve. Finally apply the above construction to the image of $\sigma_{1} * \bar{\sigma}_{3}$ under the above area preserving diffeomorphisms so that the resulting image curves are as in Figure 6 .

Because of Propositions 2.22 and 2.19 along with the structure of the Yang-Mills expectations as described in Theorem 2.14 (in particular the invariance under area preserving diffeomorphisms) it suffices to prove Theorem 2.21 in the special case where $\mathbb{G}$ is a graph in $\mathbb{R}^{2}$ such that $0 \in V(\mathbb{G})$ and $\mathbb{G}$ contains a cross lined up with the coordinate axes as in Figure 6 .

Assumption 1. For the body of this paper we will assume that $\mathbb{G}$ is a tame graph as just described.

Theorem 2.23. Let $\mathbb{G}$ and $/ /^{f}(\mathbb{G}):=/ /\left.^{f}\right|_{\mathbb{G}}$ be as in Notation 2.9 and further assume $\mathbb{G}$ contains the bonds $\left\{e_{1}, \ldots, e_{4}\right\}$ as above. If $U(/ / f(\mathbb{G}))$ is gauge invariant at 0 , then

$$
\mathbb{E}\left[\left(\nabla^{e_{1}} \cdot \nabla^{e_{2}} U\right)(/ / f(\mathbb{G}))\right]=-\frac{1}{|Q|} \mathbb{E}\left[\left(\nabla_{f(Q)}^{e_{2}} U-\nabla_{f(R Q)}^{e_{2}} U\right)\left(/ /^{f}(\mathbb{G})\right)\right] .
$$

where $R(x, y):=(x,-y)$ is reflection across the $x$-axis and $Q$ and $R Q$ are the regions shown in Figure 7 and $|Q|=m(Q)$ is the area of $Q$.

Corollary 2.24. Continuing the setup in Theorem 2.23, if $U$ is further assumed to have extended gauge invariance at 0 (see Definition 2.20), then

$$
\mathbb{E}\left[\left(\nabla^{e_{1}} \cdot \nabla^{e_{2}} U\right)(/ / f(\mathbb{G}))\right]=-\frac{1}{|Q|} \mathbb{E}\left[\left(\nabla_{f(Q)}^{e_{2}} U+\nabla_{f(R Q)}^{e_{4}} U\right)\left(/ /^{f}(\mathbb{G})\right)\right] .
$$

The proof of Theorem 2.21 will now amount to taking the limit (see Theorem 2.27) in Eq. 2.9) where $Q$ shrinks down to the the line segment, $e_{2}$. To be more precise we introduce the following notation. 


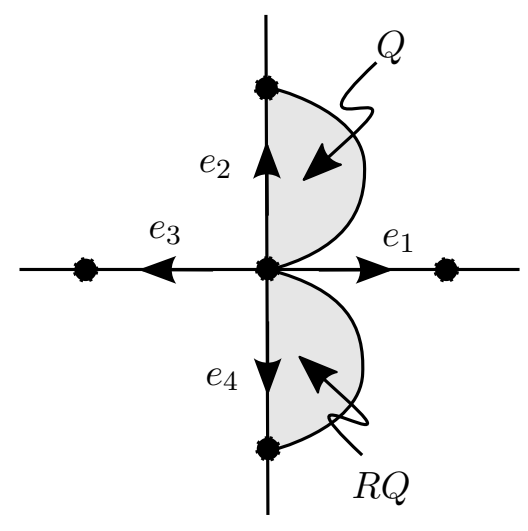

Figure 7. The regions $Q$ and $R Q$ used in Eq. (2.8).

Notation 2.25. For $0<\varepsilon$ small, let $Q_{\varepsilon}$ be a slender non-empty region as in Figure 8 and $e_{2}^{\varepsilon}$ and $e_{4}^{\varepsilon}$ be the deformations of $e_{2}$ and $e_{4}$ bounding the right side of $Q_{\varepsilon}$ and $R Q_{\varepsilon}$ respectively as in the same figure. We further suppose that $Q_{\varepsilon}$ is indexed in such a way that

$$
\varepsilon=\max \left\{x>0:[\{x\} \times \mathbb{R}] \cap Q_{\varepsilon}\right\} .
$$

Notation 2.26. Let $\mathbb{G}_{\varepsilon,+}$ be the graph $\mathbb{G}$ with $e_{2}$ replaced the deformed path $e_{2}^{\varepsilon}$ and $\mathbb{G}_{\varepsilon,-}$ be the graph $\mathbb{G}$ where $e_{4}$ is replaced by the deformed path $e_{4}^{\varepsilon}$ as shown in Figure 8 .

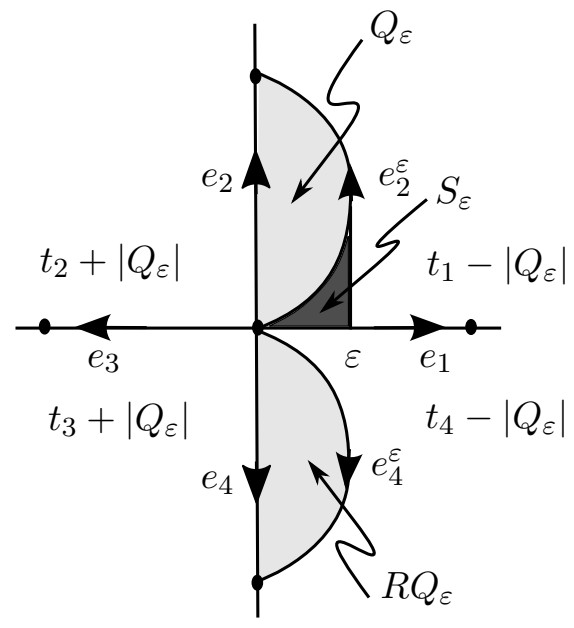

Figure 8. Deforming $e_{2}$ and $e_{4}$ in order to construct $\mathbb{G}_{+, \varepsilon}$ and $\mathbb{G}_{-, \varepsilon}$ respectively.

If $U$ is a function on $K^{\mathbb{G}}$ we may consider $U$ to also be a function on both $K^{\mathbb{G}+, \varepsilon}$ by replacing the argument $\omega\left(e_{2}\right)$ in $U$ by $\omega\left(e_{2}^{\varepsilon}\right)$. Similarly by replacing the argument $\omega\left(e_{4}\right)$ in $U$ by $\omega\left(e_{4}^{\varepsilon}\right)$ we may also view $U$ as a function on $K^{\mathbb{G}_{-, \varepsilon}}$.

Assumption 2. For the rest of this paper we now assume that $\left\{Q_{\varepsilon}\right\}$ as in Notation 2.25 is chosen in such as way that there exists $c<\infty$ so that the "shadow" region $S_{\varepsilon}$ (as indicated in Figure 8) satisfies $\left|S_{\varepsilon}\right| \leq c\left|Q_{\varepsilon}\right|$ as $\varepsilon \downarrow 0$. 
Theorem 2.27 (Loop expansion). Continuing the notation above while keeping Assumptions 1 and 2 in force, if $U: K^{\mathbb{G}} \rightarrow \mathbb{C}$ is a $C^{3}$-function, then

$$
-\mathbb{E}\left[\left(\nabla_{f\left(Q_{\varepsilon}\right)}^{e_{2}} U+\nabla_{f\left(R Q_{\varepsilon}\right)}^{e_{4}} U\right)(/ / f(\mathbb{G}))\right]=\mathbb{E}\left[U\left(/ / f\left(\mathbb{G}_{+, \varepsilon}\right)\right)-U\left(/ /^{f}\left(\mathbb{G}_{-, \varepsilon}\right)\right)\right]+O\left(\sqrt{\varepsilon}\left|Q_{\varepsilon}\right|\right) .
$$

2.3. Proof of Theorem 2.21. It is now a simple matter to use the previous results to prove Theorem 2.21.

Proof of Theorem 2.21. By Proposition 2.22 it suffices to assume the crossing configuration of $\mathbb{G}$ is at $v=0$ and lies on the coordinated axes as in Figure 6. It then follows from Corollary 2.24 and Theorem 2.27 that

$$
\begin{aligned}
\mathbb{E}\left[\left(\nabla^{e_{1}} \cdot \nabla^{e_{2}} U\right)\left(/ /^{f}(\mathbb{G})\right)\right] & =-\lim _{\varepsilon \downarrow 0} \frac{1}{\left|Q_{\varepsilon}\right|} \mathbb{E}\left[\left(\nabla_{f\left(Q_{\varepsilon}\right)}^{e_{2}} U+\nabla_{f\left(R Q_{\varepsilon}\right)}^{e_{4}} U\right)(/ / f(\mathbb{G}))\right] \\
& =\lim _{\varepsilon \downarrow 0} \frac{1}{\left|Q_{\varepsilon}\right|} \mathbb{E}\left[U\left(/ /^{f}\left(\mathbb{G}_{+, \varepsilon}\right)\right)-U\left(/ /^{f}\left(\mathbb{G}_{-, \varepsilon}\right)\right)\right] .
\end{aligned}
$$

Fixing all of the bounded areas of $\mathbb{R}^{2} \backslash \mathbb{G}$ except for those bordering the vertex $0 \in V(\mathbb{G})$, let $z$ be the function such that $z\left(t_{1}, t_{2}, t_{3}, t_{4}\right):=\mathbb{E}[U(/ / f(\mathbb{G}))]$, where $\left\{t_{i}\right\}_{i=1}^{4}$ are the areas of the faces adjoining 0 as labeled in Figure 6. [The fact that we can defined $z$ as a function of these areas and not on the shapes of the regions relies on Corollary 2.15.] We then have, see Figure 8 ,

$$
\begin{aligned}
\mathbb{E}\left[U\left(/ /{ }^{f}\left(\mathbb{G}_{+, \varepsilon}\right)\right)-U\left(/ / f\left(\mathbb{G}_{-, \varepsilon}\right)\right)\right]= & z\left(t_{1}-\left|Q_{\varepsilon}\right|, t_{2}+\left|Q_{\varepsilon}\right|, t_{3}, t_{4}\right)-z\left(t_{1}, t_{2}, t_{3}+\left|Q_{\varepsilon}\right|, t_{4}-\left|Q_{\varepsilon}\right|\right) \\
= & z\left(t_{1}-\left|Q_{\varepsilon}\right|, t_{2}+\left|Q_{\varepsilon}\right|, t_{3}, t_{4}\right)-z\left(t_{1}, t_{2}, t_{3}, t_{4}\right) \\
& -\left[z\left(t_{1}, t_{2}, t_{3}+\left|Q_{\varepsilon}\right|, t_{4}-\left|Q_{\varepsilon}\right|\right)-z\left(t_{1}, t_{2}, t_{3}, t_{4}\right)\right] .
\end{aligned}
$$

Dividing this identity by $\left|Q_{\varepsilon}\right|$ and letting $\varepsilon \downarrow 0$ shows

$$
\begin{aligned}
\lim _{\varepsilon \downarrow 0} \frac{1}{\left|Q_{\varepsilon}\right|} \mathbb{E}\left[U\left(/ / f\left(\mathbb{G}_{+, \varepsilon}\right)\right)-U\left(/ / f\left(\mathbb{G}_{-, \varepsilon}\right)\right)\right] & =\left(-\frac{\partial}{\partial t_{1}}+\frac{\partial}{\partial t_{2}}-\frac{\partial}{\partial t_{3}}+\frac{\partial}{\partial t_{4}}\right) z\left(t_{1}, t_{2}, t_{3}, t_{4}\right) \\
& =-\left(\frac{\partial}{\partial t_{1}}-\frac{\partial}{\partial t_{2}}+\frac{\partial}{\partial t_{3}}-\frac{\partial}{\partial t_{4}}\right) \mathbb{E}\left[U\left(/ /^{f}(\mathbb{G})\right)\right] .
\end{aligned}
$$

Combining Eqs. (2.11) and (2.12) completes the proof of Theorem 2.21.

\section{Heuristic ARguments}

Throughout this section, let $\mathbb{G}$ be a graph in $\mathbb{R}^{2}$ satisfying Assumption 1 , i.e. we are assuming $\mathbb{G}$ contains a cross of bonds $\left(\left\{e_{1}, e_{2}, e_{3}, e_{4}\right\}\right)$ contained in the coordinate axes as in Figure 6. In this section we will use the informal expression for the measure $\mu$ given in Eq. (1.6) and therefore many of the "results" in this section are not rigorous. We indicate the non-rigorous results by writing Meta-Theorem, Meta-Lemma, etc. Most of the results in this section will have a corresponding rigorous version in either Section 4 or Section 5 below.

\subsection{Heuristic integration by parts.}

Meta-Theorem 3.1 (Gaussian IBP). If $\Psi: \mathcal{A}_{0} \rightarrow \mathbb{C}$ and $\eta \in \mathcal{A}_{0}$, then

$$
\int_{\mathcal{A}_{0}} \Psi(A+\eta) d \mu(A)=e^{-\frac{1}{2}\left\|\partial_{y} \eta\right\|^{2}} \int_{\mathcal{A}_{0}} \Psi(A) \exp \left(\left\langle\partial_{y} A, \partial_{y} \eta\right\rangle\right) d \mu(A)
$$


and

$$
\int_{\mathcal{A}_{0}} \partial_{\eta} \Psi(A) d \mu(A)=\int_{\mathcal{A}_{0}} \Psi(A)\left\langle\partial_{y} A, \partial_{y} \eta\right\rangle d \mu(A) .
$$

Proof. Using the formal translation invariance of $\mathcal{D} A$ we find

$$
\begin{aligned}
\int_{\mathcal{A}_{0}} \Psi(A+\eta) d \mu(A) & =\frac{1}{Z} \int_{\mathcal{A}_{0}} \Psi(A+\eta) \exp \left(-\frac{1}{2}\left\|\partial_{y} A\right\|^{2}\right) \mathcal{D} A \\
& =\frac{1}{Z} \int_{\mathcal{A}_{0}} \Psi(A) \exp \left(-\frac{1}{2}\left\|\partial_{y} A-\partial_{y} \eta\right\|^{2}\right) \mathcal{D} A \\
& =e^{-\frac{1}{2}\left\|\partial_{y} \eta\right\|^{2}} \int_{\mathcal{A}_{0}} \Psi(A) \exp \left(\left\langle\partial_{y} A, \partial_{y} \eta\right\rangle\right) d \mu(A) .
\end{aligned}
$$

Replacing $\eta$ by $s \eta$ and then differentiating in $s$ at $s=0$ then gives the basic Gaussian integration by parts formula in Eq. (3.2).

Now we want to make perturbations by $\eta$ (i.e. $\eta d x$ ) where in fact $\eta(x, 0) \neq 0$ for all $x \in \mathbb{R}$. Of course we can not actually do this since this type of perturbation does not preserve the axial-gauge subspace, $\mathcal{A}_{0}$. Nevertheless we will see that Eq. (3.2) still is valid for such an $\eta$ provided $\Psi$ is $g_{s \eta}$-invariant for $s$ near 0 , see Meta-Theorem 3.10. Before proving this key meta-theorem we need to introduce the general class of perturbations to be considered.

Notation 3.2. Let $\eta_{y}: \mathbb{R}^{2} \rightarrow \mathfrak{k}$ be a bounded measurable function with compact support and set

$$
\eta(x, y):=\int_{-\infty}^{y} \eta_{y}\left(x, y^{\prime}\right) d y^{\prime} \forall(x, y) \in \mathbb{R}^{2} .
$$

Notice that for each $x \in \mathbb{R}, y \rightarrow \eta(x, y)$ is absolutely continuous and $\frac{\partial \eta}{\partial y}(x, y)=\eta_{y}(x, y)$ for a.e. $y$. Because of this observation, we will often informally describe $\eta$ as in Notation 3.2 by saying that $\eta$ is a function from $\mathbb{R}^{2}$ to $\mathfrak{k}$ such that $\eta_{y}=\partial \eta / \partial y$ is bounded and compactly supported with the understanding that $\eta$ is given as in Eq. (3.3).

Notation 3.3. Given $A: \mathbb{R}^{2} \rightarrow \mathfrak{k}$ bounded and measurable, let

$$
\bar{A}(x, y):=A(x, y)-A(x, 0) .
$$

Remark 3.4. If $\eta_{y}$ and $\eta$ are as in Notation 3.2, then for all $y \in \mathbb{R}$,

$$
\begin{aligned}
\bar{\eta}(x, y) & =\eta(x, y)-\eta(x, 0) \\
& =\int_{-\infty}^{y} \eta_{y}\left(x, y^{\prime}\right) d y^{\prime}-\int_{-\infty}^{0} \eta_{y}\left(x, y^{\prime}\right) d y^{\prime}=\int_{0}^{y} \eta_{y}\left(x, y^{\prime}\right) d y^{\prime} .
\end{aligned}
$$

The next example contains the only class of $\eta$ 's that are actually needed for the purposes of this paper.

Example 3.5. For $\xi \in \mathfrak{k}$ and $Q$ a compact region in the first quadrant as shown in Figure 7 , let $\eta$ be as in $E q$. (3.3) with

$$
\eta_{y}(x, y)=\left[1_{R Q}(x, y)-1_{Q}(x, y)\right] \cdot \xi .
$$

In this case, $\eta$ is compactly supported with $\operatorname{supp}(\eta)=\bar{Q} \cup R \bar{Q}$. 
Definition 3.6. To each $\eta$ as in Notation 3.2. let $g_{\eta} \in C(\mathbb{R}, K)$ be the absolutely continuous function satisfying the $O D E$

$$
\frac{d}{d x} g_{\eta}(x)+\eta(x, 0) g_{\eta}(x)=0 \text { for a.e. } x \text { with } g_{\eta}(0)=I \text {. }
$$

[Equation (3.4) should be interpreted in integral form as

$$
g_{\eta}(x)=I-\int_{0}^{x} \eta\left(x^{\prime}, 0\right) g_{\eta}\left(x^{\prime}\right) d x^{\prime} \forall x \in \mathbb{R} .
$$

This integral equation has a unique absolutely continuous solution.]

For our purposes, we will only deal with $g \in C\left(\mathbb{R}^{2}, K\right)$ such that $g(x, y)$ is independent of $y$ and in this setting we will identify $g$ with $g(\cdot, 0) \in C(\mathbb{R}, K)$. Thus we will abuse notation and write " $g(x, y)=g(x)$ " for any $g \in C(\mathbb{R}, K)$ and in particular apply this identification to $g_{\eta}$ of Definition 3.6.

Proposition 3.7. If $A d x$ is a connection one form, $\bar{A}$ is as in Notation 3.3, and $g_{A}$ is a in Definition 3.6 with $\eta$ replaced by $A$, then

$$
\begin{aligned}
& (A d x)^{g_{A}}(x, y)=\operatorname{Ad}_{g_{A}(x)^{-1}} \bar{A}(x, y) d x \in \mathcal{A}_{0}, \text { and } \\
& f^{(A d x)^{g_{A}}(x, y)}=\operatorname{Ad}_{g_{A}(x)^{-1}} f^{A d x}(x, y) .
\end{aligned}
$$

Proof. From the definitions we have

$$
\begin{aligned}
(A d x)^{g_{A}} & =\operatorname{Ad}_{g_{A}^{-1}} A d x+g_{A}^{-1} d g_{A}=\left[\operatorname{Ad}_{g_{A}(x)^{-1}} A(x, y)-\operatorname{Ad}_{g_{A}(x)^{-1}} A(x, 0)\right] d x \\
& =\operatorname{Ad}_{g_{A}(x)^{-1}} \bar{A}(x, y) d x
\end{aligned}
$$

which proves the first equality. The second equality follows directly from Theorem A.1 of the appendix or may be proved directly as follows,

$$
\begin{aligned}
& f^{(A d x)^{g_{A}}}(x, y)=-\partial_{y}\left[\operatorname{Ad}_{g_{A}(x)^{-1}} \bar{A}(x, y)\right]=-\operatorname{Ad}_{g_{A}(x)^{-1} \partial_{y} \bar{A}(x, y)} \\
& =-\operatorname{Ad}_{g_{A}(x)^{-1}} \partial_{y} A(x, y)=\operatorname{Ad}_{g_{A}(x)^{-1}} f^{A d x}(x, y) \text {. }
\end{aligned}
$$

Finally we introduce a class of $K$-valued functions which enable us to describe how parallel translation transforms under the perturbations, $A d x \rightarrow[(A+\eta) d x]^{g_{\eta}}$.

Definition 3.8. For $A \in \mathcal{A}_{0}$ (i.e. $\left.A d x \in \mathcal{A}_{0}\right), \eta: \mathbb{R}^{2} \rightarrow \mathfrak{k}$ as in Notation 3.2, and a horizontal path, $[a, b] \ni x \rightarrow \ell(x)=(x, y(x))$, let $[a, b] \ni x \rightarrow k_{x}(\ell)$ denote absolutely continuous function satisfying,

$$
\frac{d}{d x} k_{x}(\ell)+\left[\operatorname{Ad}_{/ / x}^{A}(\ell)^{-1} \eta(x, y(x))\right] k_{x}(\ell)=0 \text { a.e. } x \text { with } k_{0}(\ell)=I
$$

and let $\mathbf{k}^{(A, \eta)}(\ell):=k_{b}(\ell)$. [Again this equation is to be interpreted in its integral form.] 
Corollary 3.9. If $A d x \in \mathcal{A}_{0}$ (so $\left.A(x, 0) \equiv 0\right), \eta$ is as in Notation 3.2, and $g_{\eta}$ is as in Definition 3.6, then

$$
\begin{aligned}
{[(A+\eta) d x]^{g_{\eta}} } & =\operatorname{Ad}_{g_{\eta}(x)^{-1}}[A(x, y)+\bar{\eta}(x, y)] d x \in \mathcal{A}_{0}, \text { and } \\
f_{\eta}(x, y) & :=f^{[(A+\eta) d x]^{g_{\eta}}}(x, y)=\operatorname{Ad}_{g_{\eta}(x)^{-1}}\left[f(x, y)-\eta_{y}(x, y)\right] .
\end{aligned}
$$

Moreover, if $[a, b] \ni x \rightarrow \ell(x)=(x, y(x)) \in \mathbb{R}^{2}$ is a horizontal curve, then

$$
/ /[(A+\eta) d x]^{g_{\eta}}(\ell)=g_{\eta}(b)^{-1} / /_{b}^{A}(\ell) \mathbf{k}^{(A, \eta)}(\ell) g_{\eta}(a) .
$$

Proof. Most of this corollary is an easy consequence of Proposition 3.7 upon observing that

$$
\overline{A+\eta}(x, y)=A(x, y)+\bar{\eta}(x, y) \text { and }(A+\eta)(x, 0)=\eta(x, 0)
$$

and therefore; $g_{A+\eta}(x, y)=g_{\eta}(x)$,

$$
[(A+\eta) d x]^{g_{A+\eta}}=\operatorname{Ad}_{g_{\eta}(x)^{-1}}[A(x, y)+\bar{\eta}(x, y)] d x
$$

and

$$
f_{\eta}(x, y):=-\partial_{y}\left(\operatorname{Ad}_{g_{\eta}(x)^{-1}}[A(x, y)+\bar{\eta}(x, y)]\right)=\operatorname{Ad}_{g_{\eta}(x)^{-1}}\left[f(x, y)-\partial_{y} \eta(x, y)\right] .
$$

So it only remains to prove Eq. (3.8).

Differentiating the identity, $/ /{ }_{x}^{A}(\ell)^{-1} / /{ }_{x}^{A}(\ell)=I$ while making use of the Definition 1.2 shows

$$
\frac{d}{d x} / /_{x}^{A}(\ell)^{-1}=/ /_{x}^{A}(\ell)^{-1} A(x, y(x))
$$

and therefore,

$$
\begin{aligned}
\frac{d}{d x} & \left(/{ }_{x}^{A}(\ell)^{-1} / /_{x}^{(A+\eta) d x}(\ell)\right) \\
& =/ /_{x}^{A}(\ell)^{-1}(A(x, y(x))-[A(x, y(x))+\eta(x, y(x))]) / /_{x}^{(A+\eta) d x}(\ell) \\
& =-\left(\operatorname{Ad}_{\left./ /{ }_{x}^{A}(\ell)^{-1} \eta(x, y(x))\right)}\left(/ /_{x}^{A}(\ell)^{-1} / /_{x}^{(A+\eta) d x}(\ell)\right)\right.
\end{aligned}
$$

with $/ /_{x}^{A}(\ell)^{-1} / /\left.x_{x}^{(A+\eta) d x}(\ell)\right|_{x=a}=I$. By uniqueness of solutions to ODEs we conclude that

$$
/ /_{x}^{A}(\ell)^{-1} / /_{x}^{(A+\eta) d x}(\ell)=k_{x}(\ell) \Longrightarrow / /_{b}^{(A+\eta) d x}(\ell)=/ /_{b}^{A}(\ell) \mathbf{k}^{(A, \eta)}(\ell) .
$$

This equation along with Eq. A.2 of Appendix A then gives Eq. (3.8).

Meta-Theorem 3.10. If $\Psi: \mathcal{A} \rightarrow \mathbb{C}$ is an "integrable function" and $\eta=\eta d x$ is a connection one form (we do not assume $\eta(x, 0)=0$ for all $x \in \mathbb{R}$ ), then

$$
\int_{\mathcal{A}_{0}} \Psi\left((A+\eta)^{g_{\eta}}\right) d \mu(A)=e^{-\frac{1}{2}\left\|\partial_{y} \eta\right\|^{2}} \int_{\mathcal{A}_{0}} \Psi(A) \exp \left(\left\langle\partial_{y} A, \operatorname{Ad}_{g_{\eta}^{-1}} \partial_{y} \eta\right\rangle\right) d \mu(A) .
$$

Proof. By Corollary 3.9 ,

$$
(A+\eta)^{g_{\eta}}=\operatorname{Ad}_{g_{\eta}^{-1}}[A+\bar{\eta}] \in \mathcal{A}_{0},
$$


where $\bar{\eta}(x, y)=\eta(x, y)-\eta(x, 0)$. Because $g_{\eta}$ depends only on $x$ and $\operatorname{Ad}_{g_{\eta}^{-1}}$ acts isometrically on $\mathfrak{k}$ it follows that $d \mu(A)$ is invariant under $A \rightarrow \operatorname{Ad}_{g_{\eta}^{-1}} A$ and we conclude, with the aid of Eq. (3.1) and $\partial_{y} \bar{\eta}=\partial_{y} \eta$, that

$$
\begin{aligned}
\int_{\mathcal{A}_{0}} \Psi\left((A+\eta)^{g_{\eta}}\right) d \mu(A) & =\int_{\mathcal{A}_{0}} \Psi\left(\operatorname{Ad}_{g_{\eta}^{-1}}[A+\bar{\eta}]\right) d \mu(A) \\
& =e^{-\frac{1}{2}\left\|\partial_{y} \bar{\eta}\right\|^{2}} \int_{\mathcal{A}_{0}} \Psi\left(\operatorname{Ad}_{g_{\eta}^{-1}} A\right) \exp \left(\left\langle\partial_{y} A, \partial_{y} \bar{\eta}\right\rangle\right) d \mu(A) \\
& =e^{-\frac{1}{2}\left\|\partial_{y} \eta\right\|^{2}} \int_{\mathcal{A}_{0}} \Psi(A) \exp \left(\left\langle\partial_{y} \operatorname{Ad}_{g_{\eta}} A, \partial_{y} \eta\right\rangle\right) d \mu(A) \\
& =e^{-\frac{1}{2}\left\|\partial_{y} \eta\right\|^{2}} \int_{\mathcal{A}_{0}} \Psi(A) \exp \left(\left\langle\partial_{y} A, \operatorname{Ad}_{g_{\eta}^{-1}} \partial_{y} \eta\right\rangle\right) d \mu(A) .
\end{aligned}
$$

This proves Eq. (3.9).

Meta-Corollary 3.11. If $\Psi: \mathcal{A} \rightarrow \mathbb{C}$ is "smooth" and $g_{s \eta}$-invariant in the sense that $\Psi\left(B^{g_{s \eta}}\right)=$ $\Psi(B)$ for all $s$ near 0 and $B \in \mathcal{A}$ with $B=B d x(B(x, 0)$ not assumed to be zero), then Eq. 3.2) still holds, i.e.

$$
\int_{\mathcal{A}_{0}}\left(\partial_{\eta} \Psi\right)(A) d \mu(A)=\int_{\mathcal{A}_{0}} \Psi(A)\left\langle\partial_{y} A, \partial_{y} \eta\right\rangle d \mu(A)=-\int_{\mathcal{A}_{0}} \Psi(A)\left\langle f^{A}, \partial_{y} \eta\right\rangle d \mu(A) .
$$

[Note that we are substituting the assumption that $\eta \in \mathcal{A}_{0}$ by the assumption that $\Psi$ is $g_{s \eta^{-}}$ invariant.]

Meta-Proof. This result is a special case of Meta-Corollary B.32 of Appendix B. Nevertheless we will give a second "proof" here which will be closer in line with the rigorous proof of Corollary 4.12 below.

Replacing $\eta$ by $s \eta$ in Eq. (3.9) and differentiating the result leads to,

$$
\begin{aligned}
\left.\frac{d}{d s}\right|_{0} \int_{\mathcal{A}_{0}} \Psi\left((A+s \eta)^{g_{s \eta}}\right) d \mu(A) & =\int_{\mathcal{A}_{0}} \Psi(A) \exp \left(\left\langle\partial_{y} A, \partial_{y} \eta\right\rangle\right) d \mu(A) \\
& =\int_{\mathcal{A}_{0}} \Psi(A) \exp \left(\left\langle\partial_{y} A, \partial_{y} \eta\right\rangle\right) d \mu(A) .
\end{aligned}
$$

If we now further assume that $\Psi$ is $g_{s \eta}$-invariant, then

$$
\left.\frac{d}{d s}\right|_{0} \Psi\left((A+s \eta)^{g_{s \eta}}\right)=\left.\frac{d}{d s}\right|_{0} \Psi(A+s \eta)=\left(\partial_{\eta} \Psi\right)(A)
$$

which combined with Eq. (3.11) verifies Eq. 3.10).

Notation 3.12. If $\eta$ is as in Notation 3.2 and $\sigma=\left(\sigma_{1}, \sigma_{2}\right):\left[a_{\sigma}, b_{\sigma}\right] \rightarrow \mathbb{R}^{2}$ is a piecewise $C^{1}$-path, let

$$
\zeta_{\eta}^{A}(\sigma):=\int_{a_{\sigma}}^{b_{\sigma}} \operatorname{Ad}_{/ / /_{t}^{A}(\sigma)} \eta(\sigma(t)) \dot{\sigma}_{1}(t) d t \in \mathfrak{k}
$$


Lemma 3.13. If $\mathbb{G}$ is a tame graph, $U \in C^{1}\left(K^{\mathbb{G}} \rightarrow \mathbb{C}\right)$, and $\Psi(A)=U\left(/ /^{A}(\mathbb{G})\right)$, then

$$
\left(\partial_{\eta} \Psi\right)(A)=-\left(\tilde{\zeta}_{\eta}^{A} U\right)\left(/ / /^{A}(\mathbb{G})\right)=-\sum_{\sigma \in \mathbb{G}}\left(\nabla_{\zeta_{\eta}^{A}(\sigma)}^{\sigma} U\right)\left(/ /^{A}(\mathbb{G})\right)
$$

where

$$
\left(\tilde{\zeta}_{\eta}^{A} U\right)\left(/{ }^{A}(\mathbb{G})\right):=\left.\frac{d}{d s}\right|_{0} U\left(\left\{/ /{ }^{A}(\sigma) e^{s \zeta_{\eta}^{A}(\sigma)}\right\}_{\sigma \in \mathbb{G}}\right) .
$$

Proof. By Proposition A.6 of Appendix A,

$$
\begin{aligned}
\partial_{\eta}\left[A \rightarrow U\left(/ /{ }^{A}(\mathbb{G})\right)\right] & =\left.\frac{d}{d s}\right|_{0} U\left(\left\{/ /^{A}(\sigma) e^{-s \zeta_{\eta}^{A}(\sigma)}\right\}_{\sigma \in \mathbb{G}}\right) \\
& =-\left(\tilde{\zeta}_{\eta}^{A} U\right)\left(/ /{ }^{A}(\mathbb{G})\right) \\
& =-\sum_{\sigma \in \mathbb{G}}\left(\nabla_{\zeta_{\eta}^{A}(\sigma)}^{\sigma} U\right)\left(/ /^{A}(\mathbb{G})\right),
\end{aligned}
$$

where the second equality is a consequence of the chain rule.

Meta-Theorem 3.14. If $\eta$ is a $\mathfrak{k}^{\mathfrak{k}}$-valued one form on $\mathbb{R}^{2}$, and $U: K^{\mathbb{G}} \rightarrow \mathbb{C}$ is a $\left.g_{s \eta}\right|_{V(\mathbb{G})}$-invariant for $s$ near 0 , then

$$
\mathbb{E}\left[\sum_{\sigma \in \mathbb{G}}\left(\nabla_{\zeta_{\eta}^{A}(\sigma)}^{\sigma} U\right)\left(/ /^{A}(\mathbb{G})\right)\right]=\mathbb{E}\left[U\left(/ /^{A}(\mathbb{G})\right) \cdot\left\langle f^{A}, \frac{\partial \eta}{\partial y}\right\rangle\right]
$$

where $f^{A}=-\partial_{y} A$.

Proof. According to Meta-Corollary 3.11, under the given assumptions we have

$$
\begin{aligned}
\int_{\mathcal{A}_{0}} \partial_{\eta}\left[A \rightarrow U\left(/ /^{A}(\mathbb{G})\right)\right] d \mu(A) & =\int_{\mathcal{A}_{0}} \Psi(A)\left\langle\partial_{y} A, \partial_{y} \eta\right\rangle d \mu(A) \\
& =-\int_{\mathcal{A}_{0}} \Psi(A)\left\langle f^{A}, \partial_{y} \eta\right\rangle d \mu(A) .
\end{aligned}
$$

This identity along with Lemma 3.13 completes the proof.

3.2. Heuristic proof of Theorem 2.23. We are now prepared to give a heuristic proof of Theorem 2.23 as a corollary of Meta-Theorem 3.14 .

Heuristic proof of Theorem 2.23. Let $h_{y}: \mathbb{R}^{2} \rightarrow \mathbb{R}$ be a bounded measurable function with compact support,

$$
h(x, y):=\int_{-\infty}^{y} h_{y}\left(x, y^{\prime}\right) d y^{\prime},
$$

$\xi \in \mathfrak{k}$, and $\eta_{y}:=h_{y} \cdot \xi$ so that $\eta:=h \xi$. By Lemma 2.17, $\nabla_{\xi}^{e_{2}} U$ is still invariant under discrete gauge transformations, $u: V(\mathbb{G}) \rightarrow K$, such that $u(0)=I$. Thus we may apply Meta-Theorem 3.14 with $U$ replaced by $\nabla_{\xi}^{e_{2}} U$, to find

$$
\mathbb{E}\left[\sum_{\sigma \in \mathbb{G}}\left(\nabla_{\zeta_{h \xi}^{A}(\sigma)}^{\sigma}\left(\nabla_{\xi}^{e_{2}} U\right)\right)\left(/ /{ }^{A}(\mathbb{G})\right)\right]=\mathbb{E}\left[\left(\nabla_{\xi}^{e_{2}} U\right)\left(/ /^{A}(\mathbb{G})\right) \cdot\left\langle f^{A}, h_{y} \xi\right\rangle\right] .
$$


Notice that $\zeta_{h \xi}^{A}(\sigma)=0$ for $\sigma \in\left\{e_{2}, e_{4}\right\}$ since $\dot{\sigma}_{1}(t)=0$ whenever $\sigma$ is a vertical path. Let us now further assume that $h$ is supported in small neighborhood of 0 so that $\zeta_{h \xi}^{A}(\sigma)=0$ unless $\sigma \in\left\{e_{1}, e_{3}\right\}$. Since $/ /_{t}^{A}\left(e_{j}\right)=I$ for $j \in\{1,3\}$, it follows that

$$
\begin{aligned}
\zeta_{h \xi}^{A}\left(e_{1}\right) & :=\left[\int_{0}^{\infty} h(t, 0) d t\right] \xi=: \alpha_{1} \xi \text { and } \\
\zeta_{h \xi}^{A}\left(e_{3}\right) & :=\left[\int_{0}^{-\infty} h(t, 0) d t\right] \xi=: \alpha_{3} \xi
\end{aligned}
$$

and so Eq. (3.14) becomes,

$$
\mathbb{E}\left[\sum_{j=1,3} \alpha_{j}\left(\nabla_{\xi}^{e_{j}} \nabla_{\xi}^{e_{2}} U\right)\left(/ /{ }^{A}(\mathbb{G})\right)\right]=\mathbb{E}\left[\left(\nabla_{\xi}^{e_{2}} U\right)\left(/ /{ }^{A}(\mathbb{G})\right) \cdot\left\langle f^{A}, h_{y} \xi\right\rangle\right] .
$$

Summing the last equation over $\xi$ in an orthonormal basis for $\mathfrak{k}$ shows,

$$
\mathbb{E}\left[\sum_{j=1,3} \alpha_{j}\left(\nabla^{e_{j}} \cdot \nabla^{e_{2}} U\right)\left(/ /{ }^{A}(\mathbb{G})\right)\right]=\mathbb{E}\left[\left(\nabla_{\left\langle f^{A}, h_{y}\right\rangle}^{e_{2}} U\right)\left(/ /{ }^{A}(\mathbb{G})\right)\right] .
$$

Finally, we take $\eta$ as Example 3.5, i.e. $\eta=h \xi$ where

$$
h_{y}(x, y)=1_{R Q}(x, y)-1_{Q}(x, y)
$$

with $Q$ being a compact region in the first quadrant as shown in Figure 7 . With this choice,

$$
h(x, y)=\int_{-\infty}^{y}\left[1_{R Q}\left(x, y^{\prime}\right)-1_{Q}\left(x, y^{\prime}\right)\right] d y^{\prime},
$$

$\alpha_{3}=0$, and

$$
\alpha_{1}=\int_{0}^{\infty} h(x, 0) d x=\int_{0}^{\infty} d x \int_{-\infty}^{0} d y^{\prime} 1_{R Q}\left(x, y^{\prime}\right)=m(Q)=|Q| .
$$

Combining these identities with Eq. 3.15) shows

$$
|Q| \cdot \mathbb{E}\left[\left(\nabla^{e_{1}} \cdot \nabla^{e_{2}} U\right)\left(/ /^{A}(\mathbb{G})\right)\right]=\mathbb{E}\left[\left(\nabla_{\left[f^{A}(R Q)-f^{A}(Q)\right]}^{e_{2}} U\right)\left(/ /^{A}(\mathbb{G})\right)\right]
$$

which is equivalent to Eq. 2.8).

3.3. Path expansions. In order to deduce Theorem 2.27 from Theorem 2.23 we need to approximate

$$
f^{A}(Q):=-\int_{Q} \partial_{y} A(x, y) d x d y .
$$

in terms of parallel translation around the boundary of $Q$. To be more precise, let $\ell$ be the right boundary of $Q$ and for $\varepsilon>0$ (small) let $\varepsilon \ell$ and $\varepsilon Q$ be $\ell$ and $Q$ scaled by $\varepsilon$ as depicted in Figure 9 .

Notation 3.15 (Right derivatives). For $\psi \in C^{1}(K, \mathbb{C})$ and $\xi \in \mathfrak{k}$ let $\hat{\nabla}_{\xi} \psi: K \rightarrow \mathbb{C}$ be defined by,

$$
\left(\hat{\nabla}_{\xi} \psi\right)(k):=\left.\frac{d}{d t}\right|_{0} \psi\left(e^{t \xi} k\right) \text {. }
$$




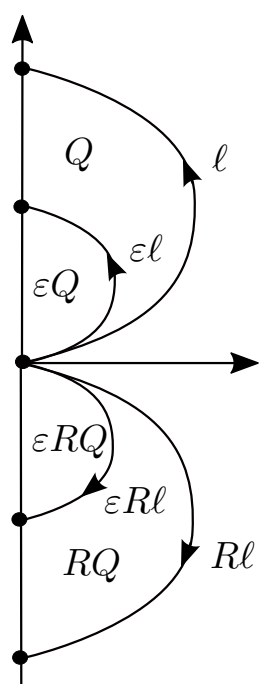

Figure 9 . Here $Q(\varepsilon Q)$ is the region bounded by the path $\ell(\varepsilon \ell)$ and the $y$-axis.

Theorem 3.16. Let $A d x$ be a smooth $t^{3}$ element of $\mathcal{A}_{0}, \varepsilon>0$, and $\varepsilon \ell$ be the path shown in Figure 9. If $\psi \in C^{2}(K, \mathbb{C})$, then

$$
\psi\left(/{ }_{1}^{A}(\varepsilon \ell)\right)=\psi(I)-\left(\nabla_{f^{A}(\varepsilon Q)} \psi\right)(e)+O\left(\varepsilon^{4}\right)
$$

where $f^{A}(\varepsilon Q)$ is as in Eq. (3.18) with $Q$ replaced by $\varepsilon Q$. Similarly if $R(x, y):=(x,-y)$ is reflection across $x$-axis, then

$$
\psi\left(/ /_{1}^{A}(\varepsilon R \ell)\right)=\psi(I)+\left(\nabla_{f^{A}(\varepsilon R Q)} \psi\right)(e)+O\left(\varepsilon^{4}\right) .
$$

Proof. Let us suppose $\ell$ is parametrized by $t \in[0,1]$ and let $g(t):=/ /{ }_{t}^{A}(\ell)$. Then $g(t)$ satisfies,

$$
\dot{g}(t)+\dot{\beta}_{\ell}(t) g(t)=0 \text { with } g(0)=I
$$

where

$$
\begin{aligned}
\beta_{\ell}(t) & :=\int_{0}^{t}(A d x)(\dot{\ell}(\tau)) d \tau=-\int_{0}^{t} d \tau \dot{\ell}_{1}(\tau) \int_{0}^{\ell_{2}(\tau)} d y f^{A}\left(\ell_{1}(\tau), y\right) \\
& =-\int_{0}^{t} d \tau \dot{\ell}_{1}(\tau) \ell_{2}(\tau) \int_{0}^{1} d s f^{A}\left(\ell_{1}(\tau), s \ell_{2}(\tau)\right) .
\end{aligned}
$$

By the fundamental theorem of calculus along with the ODE for $g$ in Eq. 3.22) we find,

$$
\begin{aligned}
\psi(g(t)) & =\psi(e)+\int_{0}^{t} \frac{d}{d \tau} \psi(g(\tau)) d \tau \\
& =\psi(e)-\int_{0}^{t}\left(\hat{\nabla}_{\dot{\beta}_{\ell}(\tau)} \psi\right)(g(\tau)) d \tau .
\end{aligned}
$$

\footnotetext{
${ }^{3}$ For example, it would suffice for $f=-\partial_{y} A$ to be continuous.
} 
Applying Eq. 3.24 with $\psi$ replaced by $\hat{\nabla}_{\dot{\beta}_{\ell}(\tau)} \psi$ shows

$$
\left(\hat{\nabla}_{\dot{\beta}_{\ell}(\tau)} \psi\right)(g(\tau))=\left(\hat{\nabla}_{\dot{\beta}_{\ell}(\tau)} \psi\right)(e)-\int_{0}^{\tau} d s\left(\hat{\nabla}_{\dot{\beta}_{\ell}(s)} \hat{\nabla}_{\dot{\beta}_{\ell}(\tau)} \psi\right)(g(s))
$$

and then substituting this expression back into Eq. (3.24) implies (taking $t=1$ ) that

$$
\psi\left(/ /{ }_{1}^{A}(\ell)\right)=\psi(e)-\left(\hat{\nabla}_{\beta_{\ell}(1)} \psi\right)(e)+\int_{0}^{1} d \tau \int_{0}^{\tau} d s\left(\hat{\nabla}_{\dot{\beta}_{\ell}(s)} \hat{\nabla}_{\dot{\beta}_{\ell}(\tau)} \psi\right)(g(s)) .
$$

Using the fact that $A d x \equiv 0$ on the $y$-axis along with Green's (or Stokes') theorem it follows that

$$
\begin{aligned}
\beta_{\ell}(1) & =\int_{0}^{1}(A d x)(\dot{\ell}(t)) d t=\int_{\partial Q} A d x \\
& =\int_{Q} F^{A d x}=\int_{Q} f^{A}(x, y) d x d y=f^{A}(Q) .
\end{aligned}
$$

Replacing $\ell$ by $\varepsilon \ell$ in Eqs. (3.23), (3.25), and (3.26) then gives Eq. (3.20). Equation (3.21) is proved similarly noting that $R$ takes counterclockwise loops to clockwise loops which changes a sign in Green's theorem. This then explains the change of sign in the gradient term when passing from Eq. (3.20) to Eq. (3.21).

3.4. Heuristic proof of Theorem 2.27. We are now almost ready to give a heuristic argument of Theorem 2.27. In order to apply Theorem 3.16, let $e_{2}^{\varepsilon}$ denote the perturbation of $e_{2}$ consisting of traversing the path $\varepsilon \ell$ followed by the straight line vertical path from $\varepsilon \ell(1)$ to $\ell(1)$ as shown in Figure 10. Similarly let $e_{4}^{\varepsilon}=R e_{2}^{\varepsilon}$ be the reflection of $e_{2}^{\varepsilon}$ so that $e_{4}^{\varepsilon}$ is a perturbation of $e_{4}$. In order to simplify notation also let $/ /{ }^{A}\left(\mathbb{G}_{ \pm}, \varepsilon\right)=\left\{/ /{ }_{1}^{A}(\sigma): \sigma \in \mathbb{G}_{ \pm}, \varepsilon\right\}$.

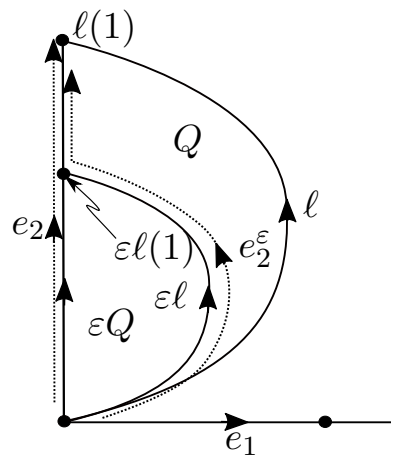

Figure 10. This figure shows the paths $e_{2}$ being deformed by the path $e_{2}^{\varepsilon}$ which consists of $\varepsilon \ell_{1}$ followed by the straight line vertical path going from $\varepsilon a$ to $a$. We will also consider the reflection of this path across the $x$-axis, $e_{4}^{\varepsilon}:=R e_{2}^{\varepsilon}$.

Corollary 3.17. Let $\mathbb{G}_{ \pm, \varepsilon}$ be the perturbations of $\mathbb{G}$ described in Notation 2.26 where $e_{2}^{\varepsilon}$ and $e_{4}^{\varepsilon}$ are the perturbations of $e_{2}$ and $e_{4}$ described above. If $A d x$ is a smooth element of $\mathcal{A}_{0}$ and $U: K^{\mathbb{G}} \rightarrow \mathbb{C}$ is a $C^{2}$-function, then

$$
U\left(/ /{ }^{A}\left(\mathbb{G}_{+, \varepsilon}\right)\right)-U\left(/ /{ }^{A}\left(\mathbb{G}_{-, \varepsilon}\right)\right)=-\left(\nabla_{f^{A}(\varepsilon Q)}^{e_{2}} U+\nabla_{f^{A}(\varepsilon R Q)}^{e_{4}} U\right)\left(/ /^{A}(\mathbb{G})\right)+O\left(\varepsilon^{4}\right)
$$


Proof. Freezing $\omega(\sigma) \in K$ for $\sigma \in \mathbb{G} \backslash\left\{e_{2}, e_{4}\right\}$ and letting $\psi_{ \pm} \in C^{2}(K, \mathbb{C})$ be chosen so that

$$
\begin{aligned}
& \psi_{+}\left(\omega\left(e_{2}\right)\right)=U(\omega) \text { with } \omega\left(e_{4}\right):=e \text { and } \\
& \psi_{-}\left(\omega\left(e_{4}\right)\right)=U(\omega) \text { with } \omega\left(e_{2}\right):=e .
\end{aligned}
$$

It then follows from Eq. (3.20) with $\psi=\psi_{+}$and Eq. (3.21) with $\psi=\psi_{-}$that

$$
\begin{aligned}
& U\left(/ /{ }^{A}\left(\mathbb{G}_{+, \varepsilon}\right)\right)=U\left(/ /{ }^{A}(\mathbb{G})\right)-\left(\nabla_{f^{A}(\varepsilon Q)}^{e_{2}} U\right)\left(/ /{ }^{A}(\mathbb{G})\right)+O\left(\varepsilon^{4}\right) \text { and } \\
& U\left(/ /{ }^{A}\left(\mathbb{G}_{-, \varepsilon}\right)\right)=U\left(/ /^{A}(\mathbb{G})\right)+\left(\nabla_{f^{A}(\varepsilon R Q)}^{e_{4}} U\right)\left(/ /^{A}(\mathbb{G})\right)+O\left(\varepsilon^{4}\right)
\end{aligned}
$$

from which Eq. (3.27) easily follows.

Heuristic Proof of Theorem 2.27. By taking expectations of Eq. (3.27) we expect

$$
\mathbb{E}\left[U\left(/ /{ }^{A}\left(\mathbb{G}_{+, \varepsilon}\right)\right)-U\left(/ /{ }^{A}\left(\mathbb{G}_{-, \varepsilon}\right)\right)\right]=-\mathbb{E}\left[\left(\nabla_{f^{A}(\varepsilon Q)}^{e_{2}} U+\nabla_{f^{A}(\varepsilon R Q)}^{e_{4}} U\right)\left(/ /{ }^{A}(\mathbb{G})\right)\right]+O\left(\varepsilon^{4}\right)
$$

which would imply Theorem 2.27 in the setting described here as $|\varepsilon Q|=\varepsilon^{2}|Q|$ so that $O\left(\varepsilon^{4}\right)=$ $O\left(|\varepsilon Q|^{2}\right)=o(|\varepsilon Q|)$.

Remark 3.18. The heuristic "proof" given above follows the spirit of the arguments in [25]. However, as we will see below the argument is too naive since the random connection one-forms $A d x$ are very rough. In reality, $f^{A}(Q)$ fluctuates on order of $\sqrt{|Q|}$ and the error term in Eqs. (3.20) and (3.21) are really $O(|Q|)$ rather than $o(|Q|)$, see Theorem 5.4 below for a precise statement. Nevertheless we will see below in subsection 5.1 that Theorem 2.27 is in fact true because of a fortuitous cancellation and the simple covariance estimate, Lemma 5.11 below.

\section{Rigorous integration By Parts}

Our goal in this section is to give a rigorous stochastic proof of Theorem 2.23. In order to prove the required integration by parts formula it is necessary to understand the distribution of a white noise after it has been transformed by rotations and translations. The key result is Corollary 4.12 which specializes the abstract white noise result reviewed in Theorem 4.5 .

4.1. Rotating and translating white noise. We begin by formally describing rotations of the white noise. If $\mathcal{O}: L^{2}\left(\mathbb{R}^{2}, m ; \mathfrak{k}\right) \rightarrow L^{2}\left(\mathbb{R}^{2}, m ; \mathfrak{k}\right)$ is any orthogonal transformation and $f$ is a white noise then we define a new white noise, $f^{\mathcal{O}}$, by

$$
\left\langle f^{\mathcal{O}}, u\right\rangle:=\langle f, \mathcal{O} u\rangle \text { for all } u \in L^{2}\left(\mathbb{R}^{2}, m ; \mathfrak{k}\right) .
$$

If $\mathcal{R}: L^{2}\left(\mathbb{R}^{2}, m ; \mathfrak{k}\right) \rightarrow L^{2}\left(\mathbb{R}^{2}, m ; \mathfrak{k}\right)$ is another isometry then

$$
\left\langle\left(f^{\mathcal{R}}\right)^{\mathcal{O}}, u\right\rangle:=\left\langle f^{\mathcal{R}}, \mathcal{O} u\right\rangle=\left\langle f, \mathcal{R O} \mathcal{O} u=\left\langle f^{\mathcal{R O}}, u\right\rangle\right.
$$

from which it follows that $(f, \mathcal{O}) \rightarrow f^{\mathcal{O}}$ is a right action. It should be clear that $f^{\mathcal{O}}$ and $f$ have the same distributions as mean zero Gaussian processes (like the white noise) are completely determined by their covariances. We will be interested here only in two special cases of this construction. The first is the transformation, $u \rightarrow \hat{u}$ and correspondingly $f \rightarrow \hat{f}$ given in Definition 2.4 above and the second is given in then next definition. 
Definition 4.1. If $g \in C\left(\mathbb{R}^{2}, K\right)$, then we let $f^{g}=f^{\operatorname{Ad}_{g}}$, i.e. $\left\langle f^{g}, u\right\rangle=\left\langle f, \operatorname{Ad}_{g} u\right\rangle$ for all $u \in L^{2}\left(\mathbb{R}^{2}, m ; \mathfrak{k}\right)$.

If the white noise were a continuous process, then we would have $f^{g}(p)=\operatorname{Ad}_{g^{-1}(p)} f(p)$ for all $p \in \mathbb{R}^{2}$. Lemma 4.3 below gives a rigorous interpretation of this informal representation of $f^{g}$.

Notation 4.2 (Oscillation semi-norms). Suppose that $B \in \mathcal{B}_{\mathbb{R}^{2}}$ is a bounded set, $g \in C^{2}\left(\mathbb{R}^{2}, K\right)$, and $\Pi \subset \mathcal{B}_{\mathbb{R}^{2}}$ denotes a finite partition of $B$. Then we let

$$
\begin{aligned}
|\Pi| & :=\max \{\operatorname{diam}(A): A \in \Pi\} \text { and } \\
\operatorname{osc}_{\Pi}(g) & :=\max _{A \in \Pi} \sup _{p, q \in A}|g(q)-g(p)| .
\end{aligned}
$$

Lemma 4.3. Let $g \in C\left(\mathbb{R}^{2}, K\right), B \in \mathcal{B}_{\mathbb{R}^{2}}$ be a bounded set, and $\left\{\Pi_{n}\right\}_{n=1}^{\infty} \subset \mathcal{B}_{\mathbb{R}^{2}}$ be a sequence of finite partitions of $B$ such that $\lim _{n \rightarrow \infty} \operatorname{osc}_{\Pi_{n}}(g)=0$, then

$$
f^{g}(B)=L^{2}(\mathbb{P})-\lim _{n \rightarrow \infty} \sum_{A \in \Pi_{n}} \operatorname{Ad}_{g\left(p_{A}\right)^{-1}} f(A)
$$

where $p_{A}$ denotes any choice of a point in $A$ for all $A \in \cup_{n=1}^{\infty} \Pi_{n}$.

Proof. Let $\xi \in \mathfrak{k}$ be fixed so that

$$
\left\langle f^{g}(B), \xi\right\rangle:=\left\langle f^{g}, \xi 1_{B}\right\rangle:=\left\langle f, \operatorname{Ad}_{g} \xi 1_{B}\right\rangle .
$$

By the assumption, $\lim _{n \rightarrow \infty} \operatorname{osc}_{\Pi_{n}}(g)=0$, along with the dominated convergence theorem,

$$
\operatorname{Ad}_{g(\cdot)} \xi 1_{B}(\cdot)=L^{2}(m)-\lim _{n \rightarrow \infty} \sum_{A \in \Pi_{n}} \operatorname{Ad}_{g\left(p_{A}\right)} \xi 1_{A} .
$$

This identity, the $\operatorname{Ad}_{g}$-invariance of the inner product on $\mathfrak{k}$, and the isometry property of the white noise (see Definition 2.1) then implies,

$$
\left\langle f, \operatorname{Ad}_{g} \xi 1_{B}\right\rangle=L^{2}(\mathbb{P})-\lim _{n \rightarrow \infty} \sum_{A \in \Pi_{n}}\left\langle f(A), \operatorname{Ad}_{g\left(p_{A}\right)} \xi\right\rangle=L^{2}(\mathbb{P})-\lim _{n \rightarrow \infty} \sum_{A \in \Pi_{n}}\left\langle\operatorname{Ad}_{g\left(p_{A}\right)^{-1}} f(A), \xi\right\rangle .
$$

As $\xi \in \mathfrak{k}$ is arbitrary, Eq. 4.1 is proved.

Recall, as mentioned after Definition 3.6, we will routinely identify $g \in C(\mathbb{R}, K)$ with $g \circ p \in$ $C\left(\mathbb{R}^{2}, K\right)$ where $p: \mathbb{R}^{2} \rightarrow \mathbb{R}$ is projection onto the first factor.

Lemma 4.4. If $g \in C(\mathbb{R}, K) \subset C\left(\mathbb{R}^{2}, K\right)$, then $(\hat{f})^{g}=\widehat{f^{g}}$ where $\hat{f}$ is as in Definition 2.4.

Proof. If $u \in L^{2}\left(\mathbb{R}^{2} ; \mathfrak{k}\right)$, then

$$
\left\langle(\hat{f})^{g}, u\right\rangle=\left\langle\hat{f}, \operatorname{Ad}_{g} u\right\rangle=\left\langle f, \widehat{\operatorname{Ad}_{g} u}\right\rangle=\left\langle f, \operatorname{Ad}_{g} \widehat{u}\right\rangle=\left\langle f^{g}, \hat{u}\right\rangle=\left\langle\widehat{f^{g}}, u\right\rangle .
$$

Theorem 4.5 (Affine change of variables). If $\alpha \in L^{2}\left(\mathbb{R}^{2} ; \mathfrak{k}\right), g \in C\left(\mathbb{R}^{2}, K\right)$, and $\psi(f)$ is a bounded measurable function of the white noise, $f$, then

$$
\mathbb{E}\left[\psi\left(f^{g}-\operatorname{Ad}_{g^{-1}} \alpha\right)\right]=\mathbb{E}\left[\psi(f) e^{-\langle f, \alpha\rangle-\frac{1}{2}\|\alpha\|^{2}}\right] .
$$

In particular the laws of $f^{g}-\operatorname{Ad}_{g^{-1}} \alpha$ and $f$ are mutually absolutely continuous relative to one another. 
Proof. By the multiplicative system theorem (see Dellacherie [10, p. 14] or Janson [21, Appendix A., p. 309]) it suffices to prove Eq. 4.2 when $\psi$ is a cylinder functions of the form,

$$
\psi(f)=\tilde{\psi}\left(\left\langle f, u_{1}\right\rangle, \ldots,\left\langle f, u_{k}\right\rangle\right)
$$

with $u_{i} \in L^{2}\left(\mathbb{R}^{2} ; \mathfrak{k}\right)$ or $u_{i} \in C_{c}^{\infty}\left(\mathbb{R}^{2} ; \mathfrak{k}\right)$ if we prefer. We may further assume that $\left\{u_{i}\right\}_{i=1}^{\infty}$ is an orthonormal basis for $L^{2}\left(\mathbb{R}^{2} ; \mathfrak{k}\right)$ in which case $\left\{\operatorname{Ad}_{g} u_{i}\right\}_{i=1}^{\infty}$ is also an orthonormal basis for $L^{2}\left(\mathbb{R}^{2} ; \mathfrak{k}\right)$. Since

$$
\begin{aligned}
\psi\left(f^{g}-\operatorname{Ad}_{g^{-1}} \alpha\right) & =\tilde{\psi}\left(\left\langle f-\alpha, \operatorname{Ad}_{g} u_{1}\right\rangle, \ldots,\left\langle f-\alpha, \operatorname{Ad}_{g} u_{k}\right\rangle\right) \\
& =\tilde{\psi}\left(\left\langle f, \operatorname{Ad}_{g} u_{1}\right\rangle-\left\langle\alpha, \operatorname{Ad}_{g} u_{1}\right\rangle, \ldots,\left\langle f, \operatorname{Ad}_{g} u_{k}\right\rangle-\left\langle\alpha, \operatorname{Ad}_{g} u_{k}\right\rangle\right)
\end{aligned}
$$

it follows by a finite dimensional change of variables and the fact that $\left\{\left\langle f, \operatorname{Ad}_{g} u_{i}\right\rangle\right\}_{i=1}^{\infty}$ are i.i.d. standard normal random variables that

$$
\begin{aligned}
\mathbb{E}\left[\psi\left(f^{g}-\operatorname{Ad}_{g^{-1}}\right)\right] & =\mathbb{E}\left[\tilde{\psi}\left(\left\langle f, \operatorname{Ad}_{g} u_{1}\right\rangle-\left\langle\alpha, \operatorname{Ad}_{g} u_{1}\right\rangle, \ldots,\left\langle f, \operatorname{Ad}_{g} u_{k}\right\rangle-\left\langle\alpha, \operatorname{Ad}_{g} u_{k}\right\rangle\right)\right] \\
& =\mathbb{E}\left[\tilde{\psi}\left(\left\langle f, \operatorname{Ad}_{g} u_{1}\right\rangle, \ldots,\left\langle f, \operatorname{Ad}_{g} u_{k}\right\rangle\right) Z_{\tilde{k}}\right]
\end{aligned}
$$

where for any $\tilde{k} \geq k$,

$$
Z_{\tilde{k}}=\exp \left(-\sum_{j=1}^{\tilde{k}}\left[\left\langle f, \operatorname{Ad}_{g} u_{j}\right\rangle\left\langle\alpha, \operatorname{Ad}_{g} u_{j}\right\rangle-\frac{1}{2}\left|\left\langle\alpha, \operatorname{Ad}_{g} u_{j}\right\rangle\right|^{2}\right]\right)
$$

Using

$$
L^{\infty-}-\lim _{\tilde{k} \uparrow \infty} Z_{\tilde{k}}=\exp \left(-\langle f, \alpha\rangle-\frac{1}{2}\|\alpha\|^{2}\right)
$$

we may pass to the limit as $\tilde{k} \rightarrow \infty$ in Eq. (4.3) to arrive at Eq. 4.2 .

Proposition 4.6. Suppose that $f$ is a $\mathfrak{k}$-valued white noise, $[a, b] \ni t \rightarrow \ell(t):=(t, y(t))$ is $a$ horizontal curve in $\mathbb{R}^{2}$, and $\left\{M_{t}^{f}(\ell)\right\}_{t \in[a, b]}$ is the $\mathfrak{k}$-valued martingale as defined in Definition 2.6. If $g \in C(\mathbb{R}, K)$ such that $g(0)=I$, then $d M_{t}^{f^{g}}(\ell)=\operatorname{Ad}_{g^{-1}(t)} d M_{t}^{f}(\ell)$, i.e.

$$
M_{t}^{f^{g}}(\ell)=\int_{a}^{t} \operatorname{Ad}_{g^{-1}(\tau)} d M_{\tau}^{f}(\ell)
$$

where the latter integral is a Itô (or essentially Wiener) stochastic integral. 
Proof. Let $\Pi=\left\{a=t_{0}<t_{1}<\cdots<t_{n}=t\right\}$ denote a partition of $[a, t]$. By Lemma 4.3 ,

$$
\begin{aligned}
M_{t}^{\ell}\left(f^{g}\right) & =-\hat{f}^{g}\left(R_{t}^{\ell}\right)=L^{2}(\mathbb{P})-\lim _{|\Pi| \rightarrow 0} \sum_{j=1}^{n}-\operatorname{Ad}_{g\left(t_{j-1}\right)} \hat{f}\left(R_{t_{j}}^{\ell} \backslash R_{t_{j-1}}^{\ell}\right) \\
& =L^{2}(\mathbb{P})-\lim _{|\Pi| \rightarrow 0} \sum_{j=1}^{n}-\operatorname{Ad}_{g\left(t_{j-1}\right)}\left[\hat{f}\left(R_{t_{j}}^{\ell}\right)-\hat{f}\left(R_{t_{j-1}}^{\ell}\right)\right] \\
& =L^{2}(\mathbb{P})-\lim _{|\Pi| \rightarrow 0} \sum_{j=1}^{n} \operatorname{Ad}_{g\left(t_{j-1}\right)}\left[M_{t_{j}}^{\ell}(f)-M_{t_{j-1}}^{\ell}(f)\right] \\
& =\int_{a}^{t} \operatorname{Ad}_{g(\tau)} d M_{\tau}^{f}(\ell) .
\end{aligned}
$$

4.2. Perturbations of $f, M^{f}$, and $/ /{ }^{f}$. We start by making precise the perturbation, $f_{\eta}$, of $f$ which was introduced informally in Eq. 3.7.

Definition 4.7. For $\eta, \eta_{y}: \mathbb{R}^{2} \rightarrow \mathfrak{k}$ as in Notation 3.2, let

$$
f_{\eta}:=f^{g_{\eta}}-\operatorname{Ad}_{g_{\eta}^{-1}} \eta_{y}
$$

where $g_{\eta} \in C(\mathbb{R} \rightarrow K)$ is the solution to the ODE in Eq. 3.4) in Definition 3.6.

Theorem 4.8 (Martingale perturbations). Let $[a, b] \ni x \rightarrow \ell(x):=(x, y(x))$ be a horizontal curve in $\mathbb{R}^{2}, f$ be a $\mathfrak{k}$-valued white noise, $\eta, \eta_{y}: \mathbb{R}^{2} \rightarrow \mathfrak{k}$ be as in Notation 3.2, and $\left\{M_{t}^{f}(\ell)\right\}_{t \in[a, b]}$ be the martingale defined in Definition 2.6. Then $\left\{M_{t}^{f_{\eta}}(\ell)\right\}_{t \in[a, b]}$ is the semi-martingale given by the following Itô integrals;

$$
M_{t}^{f_{\eta}}(\ell)=\int_{a}^{t} \operatorname{Ad}_{g_{\eta}(x)^{-1}} d M_{x}^{f}(\ell)+\int_{a}^{t} \operatorname{Ad}_{g_{\eta}(x)^{-1}} \bar{\eta}(x, y(x)) d x .
$$

Alternatively, the differential form of Eq. 4.5 is

$$
d M_{x}^{f_{\eta}}(\ell)=\operatorname{Ad}_{g_{\eta}(x)^{-1}}\left[d M_{x}^{f}(\ell)+\bar{\eta}(x, y(x)) d x\right] .
$$

[Recall from Notation 3.3 and Remark 3.4 that

$$
\left.\bar{\eta}(x, y):=\eta(x, y)-\eta(x, 0)=\int_{0}^{y} \eta_{y}\left(x, y^{\prime}\right) d y^{\prime} .\right]
$$

Proof. Let us first observe that it makes sense to replace $f$ by $f_{\eta}$ in $M_{x}^{f}(\ell)$ since (as a consequence of Theorem 4.5 the laws of $f$ and $f_{\eta}$ are mutually absolutely continuous relative to one another. 
The identity in Eq. 4.5 is now a matter of unwinding the definitions;

$$
\begin{aligned}
M_{t}^{f_{\eta}}(\ell) & =-\hat{f}_{\eta}\left(R_{t}^{\ell}\right)=-\widehat{f^{g_{\eta}}}\left(R_{t}^{\ell}\right)+\int_{R_{t}^{\ell}} \operatorname{Ad}_{g_{\eta}^{-1}(x)} \operatorname{sgn}(y) \eta_{y}(x, y) d x d y \\
& =M_{t}^{f^{g_{\eta}}}(\ell)+\int_{a}^{t} d x \int_{0}^{y(x)} d y \operatorname{Ad}_{g_{\eta}^{-1}(x)} \eta_{y}(x, y) \\
& =M_{t}^{f^{g_{\eta}}}(\ell)+\int_{a}^{t} \operatorname{Ad}_{g_{\eta}(x)^{-1}} \bar{\eta}(x, y(x)) d x
\end{aligned}
$$

The desired result now follows from this equation along with Proposition 4.6.

We now introduce the white noise variant of Definition 3.8 .

Definition 4.9. If $[a, b] \ni x \rightarrow \ell(x)=(x, y(x))$ is a horizontal path, let $k_{x}(\ell)$ denote the solution to the $O D E$,

$$
\frac{d}{d x} k_{x}(\ell)+\left[\operatorname{Ad}_{/ / x}^{f}(\ell)^{-1} \eta(x, \ell(x))\right] k_{x}(\ell)=0 \text { with } k_{0}=I
$$

Further let $\mathbf{k}^{\eta}(\ell)=k_{b}(\ell)$.

Although suppressed from the notation, the functions, $x \rightarrow k_{x}(\ell)$ are in general random and depend on the white noise $f$ through the dependence of Eq. 4.7$)$ on $\left\{/ / f_{x}^{f}(\ell)\right\}_{x \in[a, b]}$. The key result of this section is the following stochastic analogue of Eq. 3.8 of Corollary 3.9.

Theorem 4.10 (Perturbed parallel translation). If $\eta$ is as above and $[a, b] \ni x \rightarrow \ell(x)=(x, y(x))$ is a horizontal path, then

$$
/ / f_{\eta}(\ell)=g_{\eta}(b)^{-1} / / f(\ell) \mathbf{k}^{\eta}(\ell) g_{\eta}(a) .
$$

[Again, it makes sense to replace $f$ by $f_{\eta}$ in $/ / f(\ell)$ since (as a consequence of Theorem 4.5) the laws of $f$ and $f_{\eta}$ are mutually absolutely continuous relative to one another.]

Proof. From Definition 3.6 and Eq. (4.6),

$$
\begin{aligned}
\delta\left[g_{\eta}(x) / f_{x}^{f_{\eta}}(\ell)\right] & =-\eta(x, 0) g_{\eta}(x) / / f_{x}^{f_{\eta}}(\ell) d x-g_{\eta}(x) \delta M_{x}^{f_{\eta}}(\ell) / /_{x}^{f_{\eta}}(\ell) \\
& =-\eta(x, 0) d x g_{\eta}(x) / /_{x}^{f_{\eta}}(\ell)-g_{\eta}(x) \operatorname{Ad}_{g_{\eta}(x)^{-1}}\left[\delta M_{x}^{f}(\ell)+\bar{\eta}(x, y(x)) d x\right] / /_{x}^{f_{\eta}}(\ell) \\
& =-\left[\delta M_{x}^{f}(\ell)+\eta(x, y(x)) d x\right] g_{\eta}(x) / /_{x}^{f_{\eta}}(\ell) .
\end{aligned}
$$

As in the proof of Corollary 3.9 , taking the Stratonovich differential of the identity, $/ /{ }_{x}^{f}(\ell)^{-1} / / f_{x}^{f_{\eta}}(\ell)=$ $I$, shows

$$
\delta / /_{x}^{f}(\ell)^{-1}=/ /_{x}^{f}(\ell)^{-1} \delta M_{x}^{f}(\ell) .
$$

Combining the previous two equations, it follows that $V_{x}:=/ /_{x}^{f}(\ell)^{-1} g_{\eta}(x) / / f_{x}^{f_{\eta}}(\ell)$ satisfies,

$$
\begin{aligned}
\delta V_{x} & =/ /_{x}^{f}(\ell)^{-1} \delta M_{x}^{f}(\ell) g_{\eta}(x) / /_{x}^{f_{\eta}}(\ell)-/{ }_{x}^{f}(\ell)^{-1}\left[\delta M_{x}^{f}(\ell)+\eta(x, y(x)) d x\right] g_{\eta}(x) /{ }_{x}^{f_{\eta}}(\ell) \\
& =-/{ }_{x}^{f}(\ell)^{-1} \eta(x, y(x)) g_{\eta}(x) / /_{x}^{f_{\eta}}(\ell) d x=-\left[\operatorname{Ad}_{/ /{ }_{x}^{f}(\ell)^{-1}} \eta(x, y(x))\right] V_{x} d x,
\end{aligned}
$$


i.e. $V_{x}$ satisfies the same ODE that $k_{x} g_{\eta}(a)$ satisfies. Therefore by the uniqueness of solutions to ODEs, $V_{x}=k_{x} g_{\eta}(a)$ and hence

$$
/ / f_{x}^{f_{\eta}}(\ell)=g_{\eta}(x)^{-1} /{ }_{x}^{f}(\ell) V_{x}=g_{\eta}(x)^{-1} /{ }_{x}^{f}(\ell) k_{x} g_{\eta}(a) .
$$

4.3. Proof of Theorem 2.23. For the proof of Theorem 2.23, we first need to deduce the required integration by parts formulas from the results in the previous subsection.

Corollary 4.11. Let $\mathbf{k}^{\eta}(\sigma)$ be as in Definition 4.9 and $g_{\eta} \in K$ be as in Definition 3.6. If $U: K^{\mathbb{G}} \rightarrow \mathbb{R}$ is a smooth function which is discrete gauge invariant under the action determined by $g_{\eta}$ (i.e. $u(v)=g_{\eta}(v)$ for all $v \in V(\mathbb{G})$ ), then

$$
\mathbb{E}\left[U\left(\left\{/ / f(\sigma) \mathbf{k}^{\eta}(\sigma)\right\}_{\sigma \in \mathbb{G}}\right)\right]=\mathbb{E}\left[U(/ / f(\mathbb{G})) \cdot \exp \left(-\left\langle f, \eta_{y}\right\rangle-\frac{1}{2}\left\|\eta_{y}\right\|^{2}\right)\right] .
$$

Proof. Using the gauge invariance assumption along with Theorem 4.10 we find,

$$
\begin{aligned}
\mathbb{E}\left[U\left(\left\{/ /^{f}(\sigma) \mathbf{k}^{\eta}(\sigma)\right\}_{\sigma \in \mathbb{G}}\right)\right] & =\mathbb{E}\left[U\left(\left\{g_{\eta}\left(\sigma_{f}\right)^{-1} / / f(\sigma) \mathbf{k}^{\eta}(\sigma) g_{\eta}\left(\sigma_{i}\right)\right\}_{\sigma \in \mathbb{G}}\right)\right] \\
& =\mathbb{E}\left[U \left(\left\{/ /^{\left.\left.\left.f^{g_{\eta}}-\operatorname{Ad}_{g_{\eta}^{-1} \eta_{y}}(\sigma)\right\}_{\sigma \in \mathbb{G}}\right)\right] .}\right.\right.\right.
\end{aligned}
$$

This equation along with Theorem 4.5 then completes the proof.

The next corollary is a rigorous version of Theorem 3.14 above.

Corollary 4.12 (Key IBP formula). Continuing the notation and assumptions of Corollary 4.11 and further letting

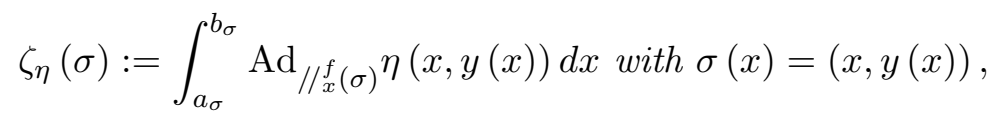

we have the integration by parts formula,

$$
\mathbb{E}\left[\sum_{\sigma \in \mathbb{G}}\left(\nabla_{\zeta_{\eta}(\sigma)}^{\sigma} U\right)\left(/^{f}(\mathbb{G})\right)\right]=\mathbb{E}\left[U\left(/ /^{f}(\mathbb{G})\right) \cdot\left\langle f, \eta_{y}\right\rangle\right] .
$$

Proof. Let $\mathbf{k}^{s \eta}(\sigma)$ be defined as in Definition 4.9 with $\eta$ replaced by $s \eta$ in which case Eq. (4.9) reads,

$$
\mathbb{E}\left[U\left(\left\{/^{f}(\sigma) \mathbf{k}^{s \eta}(\sigma)\right\}_{\sigma \in \mathbb{G}}\right)\right]=\mathbb{E}\left[U(/ / f(\mathbb{G})) \cdot \exp \left(-s\left\langle f, \eta_{y}\right\rangle-\frac{s^{2}}{2}\left\|\eta_{y}\right\|^{2}\right)\right]
$$

Differentiating this equation with respect to $s$ then gives the integration by parts formula,

$$
\mathbb{E}\left[\left.\frac{d}{d s}\right|_{0} U\left(\left\{/ f^{f}(\sigma) \mathbf{k}^{s \eta}(\sigma)\right\}_{\sigma \in \mathbb{G}}\right)\right]=-\mathbb{E}\left[U(/ / f(\mathbb{G})) \cdot\left\langle f, \eta_{y}\right\rangle\right] .
$$

Letting $\kappa_{x}^{\sigma}:=\left.\frac{d}{d s}\right|_{0} k_{x}^{s \eta}(\sigma)$ we find, by differentiating the ODE,

$$
\frac{d}{d x} k_{x}^{s \eta}=-s\left(u_{x}^{-1} \eta(x, y(x)) u_{x}\right) k_{x}^{s \eta}
$$


for $k_{x}^{s \eta}$ at $s=0$ that

$$
\begin{aligned}
\frac{d}{d x} \kappa_{x}^{\sigma} & :=\left.\frac{d}{d s}\right|_{0}\left[-s\left(u_{x}^{-1} \eta(x, y(x)) u_{x}\right) k_{x}^{s \eta}\right] \\
& =-\operatorname{Ad}_{u_{x}^{-1} \eta}(x, y(x))=-\operatorname{Ad}_{/ / x}(\sigma) \eta(x, y(x))
\end{aligned}
$$

and so

$$
\left.\frac{d}{d s}\right|_{0} / /^{f}(\sigma) \cdot \mathbf{k}^{s \eta}(\sigma)=-/ / f(\sigma) \cdot \int_{a}^{b} \operatorname{Ad}_{/ / x}^{f}(\sigma) \eta(x, y(x)) d x=-/ / f(\sigma) \cdot \zeta_{\eta}(\sigma) .
$$

Therefore

$$
\left.\frac{d}{d s}\right|_{0} U\left(\left\{/ / f(\sigma) \mathbf{k}^{s \eta}(\sigma)\right\}_{\sigma \in \mathbb{G}}\right)=-\left(\tilde{\zeta}_{\eta} U\right)\left(/ /^{f}(\mathbb{G})\right)=-\sum_{\sigma \in \mathbb{G}}\left(\nabla_{\zeta_{\eta}(\sigma)}^{\sigma} U\right)(/ / f(\mathbb{G}))
$$

which combined with Eq. 4.12 gives Eq. 4.11).

We are now ready to prove Theorem 2.23 .

Proof of Theorem 2.23. Let $\xi \in \mathfrak{k}:=\operatorname{Lie}(K)$ and $h$ be the function defined in Eq. (3.17) with $Q$ sufficiently small. Following the heuristic proof in Subsection 3.2 , we then find $\zeta_{\eta}(\sigma)=0$ unless $\sigma=e_{1}$ and for $\sigma=e_{1}$,

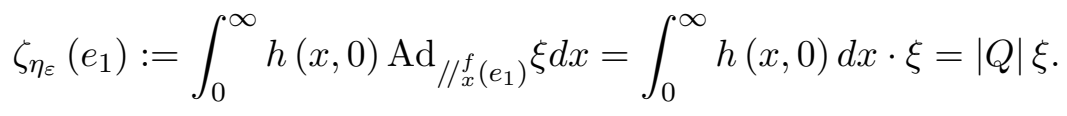

By Lemma 2.17, $\nabla_{\xi}^{e_{2}} U$ is still invariant under discrete gauge transformations, $u: V(\mathbb{G}) \rightarrow K$, such that $u(0)=I$. Hence, applying Corollary 4.12 with $U$ replaced by $\nabla_{\xi}^{e_{2}} U$ shows,

$$
\begin{aligned}
|Q| \mathbb{E}\left[\nabla_{\xi}^{e_{1}} \nabla_{\xi}^{e_{2}} U\left(/^{f}(\mathbb{G})\right)\right] & =\mathbb{E}\left[\tilde{\zeta}_{\eta} \nabla_{\xi}^{e_{2}} U\left(/ /{ }^{f}(\mathbb{G})\right)\right] \\
& =\mathbb{E}\left[\left(\nabla_{\xi}^{e_{2}} U\right)\left(/^{f}(\mathbb{G})\right) \cdot\left\langle f,\left(\partial_{y} h\right) \xi\right\rangle\right] \\
& =\mathbb{E}\left[\left(\nabla_{\xi}^{e_{2}} U\right)\left(/^{f}(\mathbb{G})\right) \cdot\langle f(R Q)-f(Q), \xi\rangle_{\mathfrak{k}}\right]
\end{aligned}
$$

wherein we have used Eq. (3.16) for the last equality. Summing this equation on $\xi \in \beta$ (an orthonormal basis for $\mathfrak{k}$ ) then completes the proof of Eq. (2.8).

\section{LOOP EXPANSION OF PARALLEL TRANSLATION}

As above, $f$ is the $\mathfrak{k}$ - valued white noise on $\mathbb{R}^{2}$. Let $u, v:[0,1] \rightarrow \mathbb{R}$ be continuous functions such that either $0 \leq u(t) \leq v(t)$ or $0 \geq u(t) \geq v(t), \sigma(t)=(t, u(t))$ and $\gamma(t)=(t, v(t))$ be the associated horizontal paths, and $Q_{t}$ be the region bounded by $y=u(t), y=v(t), x=0$, and $x=t$, see Figure 11. Further let $M_{t}^{f}(\gamma)$ and $M_{t}^{f}(\sigma)$ be the associated martingales,

$$
b_{t}:=M_{t}^{f}(\gamma)-M_{t}^{f}(\sigma)=-\hat{f}\left(Q_{t}\right)
$$

and

$$
a_{t}^{\gamma}:=\int_{0}^{t}|v(\tau)| d \tau, a_{t}^{\sigma}:=\int_{0}^{t}|u(\tau)| d \tau, \text { and } a_{t}:=\int_{0}^{t}|v(\tau)-u(\tau)| d \tau=\left|Q_{t}\right| .
$$

In this section, we will often make use of the Burkholder-Davis-Gundy inequalities in the form we now describe. Let $V$ be a finite dimensional inner product space, $n \in \mathbb{N}, L\left(\mathfrak{k}^{n}, V\right)$ be the 


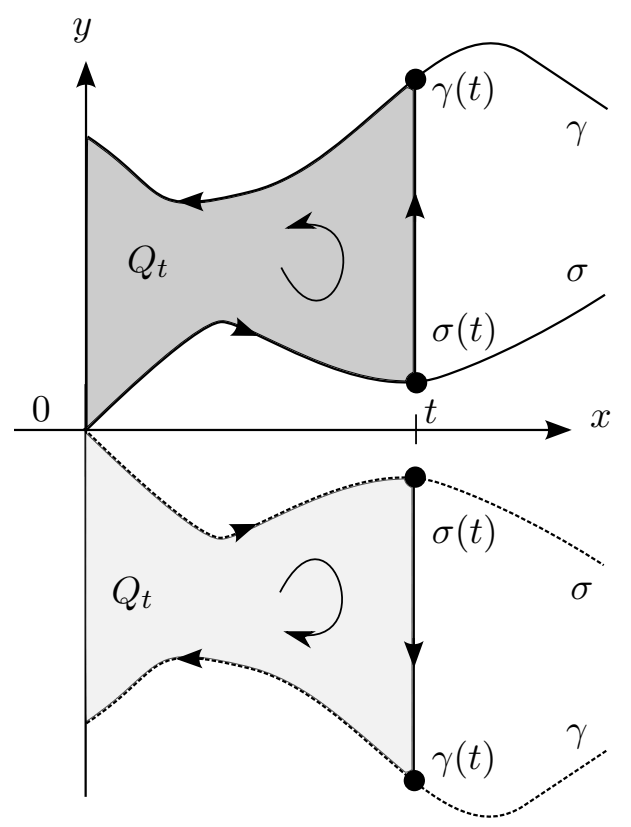

Figure 11. Both scenarios on the ordering of the functions $u$ and $v$ are depicted in this picture. The reader should refer to the figures in the upper (lower) half plane when $0 \leq u \leq v(v \leq u \leq 0)$.

linear transformations from $\mathfrak{k}^{n}$ to $V,\left\{\beta_{t}\right\}_{t \geq 0}$ be a $\mathfrak{k}^{n}$-valued continuous square integrable martingale with with independent increments, and $\left\{\langle\beta\rangle_{t}\right\}_{t \geq 0}$ be the quadratic variation of $\left\{\beta_{t}\right\}_{t \geq 0}$. If $\left\{u_{t} \in L\left(\mathfrak{k}^{n}, V\right)\right\}_{t>0}$ is an adapted continuous process and $\left\{\alpha_{t}\right\}_{t>0}$ is an increasing process dominating $\langle\beta\rangle_{t}$ (i.e. there exists $c<\infty$ such that $d\langle\beta\rangle_{t} \leq c d \alpha_{t}$ for all $t$ ), then there exists a constant $C_{p}<\infty$ depending only on $p, c, n$, and $V$, such that

$$
\left\|\int_{0}^{T} w_{\tau} d \beta_{\tau}\right\|_{p} \leq C_{p} \sqrt{\int_{0}^{T}\left\|w_{\tau}\right\|_{p}^{2} d \alpha_{\tau}} \forall 0<T<\infty .
$$

To prove this estimate we may assume $\int_{0}^{T}\left\|w_{\tau}\right\|_{p}^{2} d \alpha_{\tau}<\infty$ since otherwise Eq. 5.2 is trivial. Under this assumption, $M_{t}=\int_{0}^{t} w_{\tau} d \beta_{\tau}$ is a square integrable $V$-valued martingale satisfying, $\langle M\rangle_{T} \leq c \int_{0}^{T}\left|w_{\tau}\right|^{2} d \alpha_{\tau}$ where $\left|w_{\tau}\right|$ is an appropriate Hilbert-Schmidt norm of $w_{\tau}$. If we let $M_{T}^{*}=$ $\max _{0 \leq t \leq T}\left|M_{t}\right|$, then the Burkholder-Davis-Gundy inequalities state that $\left\|M_{T}^{*}\right\|_{p} \asymp\left\|\sqrt{\langle M\rangle_{T}}\right\|_{p}$, i.e. there exist finite constants, $c_{p}$ and $C_{p}$ such that

$$
c_{p}\left\|\sqrt{\langle M\rangle_{T}}\right\|_{p} \leq\left\|M_{T}^{*}\right\|_{p} \leq C_{p}\left\|\sqrt{\langle M\rangle_{T}}\right\|_{p} .
$$


From the second inequality we then have,

$$
\begin{aligned}
\left\|\int_{0}^{T} w_{\tau} d \beta_{\tau}\right\|_{p} \lesssim\left\|\sqrt{\int_{0}^{T}\left|w_{\tau}\right|^{2} d \alpha_{\tau}}\right\|_{p} & =\left\|\int_{0}^{T}\left|w_{\tau}\right|^{2} d \alpha_{\tau}\right\|_{p / 2}^{1 / 2} \\
& \leq \sqrt{\int_{0}^{T}\left\|\left|w_{\tau}\right|^{2}\right\|_{p / 2} d \alpha_{\tau}}=\sqrt{\int_{0}^{T}\left\|w_{\tau}\right\|_{p}^{2} d \alpha_{\tau}}
\end{aligned}
$$

which proves Eq. (5.2).

Example 5.1. Let $\left\{b_{t}\right\}_{t \geq 0}$ be as in Eq. (5.1), $n=1, \beta_{t}=b_{t}$, and $\alpha_{t}=a_{t}=\left|Q_{t}\right|$ so that $d\langle b\rangle_{t}=\operatorname{dim} \mathfrak{k} \cdot d \alpha_{t}$. Taking $V=\mathfrak{k} \otimes \mathfrak{k}$ and $w_{t}=b_{t} \otimes(\cdot)$ in Eq. (5.2) then gives,

$$
\left\|\int_{0}^{T} b_{t} \otimes d b_{t}\right\|_{p} \lesssim \sqrt{\int_{0}^{T}\left\|b_{\tau}\right\|_{p}^{2} d \alpha_{\tau}} \lesssim \sqrt{\int_{0}^{T} \alpha_{\tau} d \alpha_{\tau}}=\sqrt{\frac{1}{2} \alpha_{T}^{2}}=\sqrt{\frac{1}{2}} a_{T},
$$

wherein we have used $\left\|b_{\tau}\right\|_{p}^{2} \asymp\left\|b_{\tau}\right\|_{2}^{2}=\operatorname{dim} \mathfrak{k} \cdot \alpha_{\tau}$ because $b_{\tau}$ is a Gaussian random vector. Similarly one shows $\left\|\int_{0}^{T} d b_{t} \otimes b_{t}\right\|_{p} \lesssim a_{T}$.

Recall that $K$ is assumed to be a matrix Lie group imbedded $\mathbb{C}^{D \times D}$ - the space of $D \times D$ matrices with complex entries. For a function, $\psi: \mathbb{C}^{D \times D} \rightarrow \mathbb{C}$, let $D^{j} \psi=\psi^{(j)}$ denote the $j^{\text {th }}$ differential of $\psi$ as function on $\mathbb{C}^{D \times D}$ thought of as a real vector space. Also let $\Delta_{K}$ be the Laplacian on $K$, i.e.

$$
\left(\Delta_{K} \psi\right)(k)=\left.\sum_{\xi \in \beta} \frac{d^{2}}{d t^{2}}\right|_{0} \psi\left(k e^{t \xi}\right)
$$

where $\beta$ is any orthonormal basis for $\mathfrak{k}$.

Lemma 5.2. If $\psi: \mathbb{C}^{D \times D} \rightarrow \mathbb{C}$ is a smooth function in a neighborhood of $I \in K \subset \mathbb{C}^{D \times D}$, then

$$
\left(\Delta_{K} \psi\right)(I)=\sum_{\xi \in \beta}\left[\psi^{\prime \prime}(I) \xi \otimes \xi+\psi^{\prime}(I) \xi^{2}\right]=\sum_{\xi \in \beta} \psi^{\prime \prime}(I) \xi \otimes \xi+\psi^{\prime}(I) \kappa,
$$

where again $\beta$ is any orthonormal basis for $\mathfrak{k}$ and $\kappa:=\sum_{\xi \in \beta} \xi^{2}$ is the Casmir matrix. [As usual, it easily verified that $\Delta_{K}$ and the matrix $\kappa$ are independent of the choice of orthonormal basis of $\mathfrak{k}$.$] .$

Proof. For $\xi \in \mathfrak{k}$ we have

$$
\left(\tilde{\xi}^{2} \psi\right)(I)=\left.\frac{d^{2}}{d t^{2}}\right|_{0} \psi\left(e^{t \xi}\right)=\left.\frac{d}{d t}\right|_{0}\left[\psi^{\prime}\left(e^{t \xi}\right) \xi e^{t \xi}\right]=\psi^{\prime \prime}(I) \xi \otimes \xi+\psi^{\prime}(I) \xi^{2} .
$$

Summing the above identity on $\xi \in \beta$ gives Eq. (5.3).

To simplify notation in the statements and the proofs to follow we will adopt the following notation.

Notation 5.3 (O-notation). If $\left\{A_{t}\right\}_{0<t<\delta}$ is a collection of random variables and $(0, \delta) \ni t \rightarrow$ $a_{t} \in(0, \infty)$ is a positive function, we write $A_{t}=O\left(a_{t}^{3 / 2}\right)$ provided; for all $1 \leq p<\infty$ there exists $C=C(p, a, A)<\infty$ such that $\left\|A_{t}\right\|_{p} \leq C a_{t}$ for $0<t<\delta$. 
Theorem 5.4 (Loop Expansions). Let $u, v, \sigma, \tau$, and $Q_{t}$ be as described at the start of this section, see Figure 11 and let $\partial Q_{t}$ denote the path traversing the boundary of $Q_{t}$ in the counter-clockwise (clockwise) direction starting at $0 \in \mathbb{R}^{2}$ when $0 \leq u \leq v(v \leq u \leq 0)$. Then

$$
/^{f}\left(\partial Q_{t}\right)=I-\hat{f}\left(Q_{t}\right)+\frac{1}{2} \kappa a_{t}+\mathcal{R}_{t}+R_{t}
$$

where $\mathcal{R}_{t}$ and $R_{t}$ are matrix valued random variables satisfying;

$$
\mathbb{E} \mathcal{R}_{t}=0, \quad \mathcal{R}_{t}=O_{p}\left(\sqrt{a_{t}^{\gamma} a_{t}}\right), \text { and } R_{t}=O_{p}\left(a_{t}^{3 / 2}\right)
$$

Proof. Let $h_{t}=/ /_{t}^{f}(\sigma)$ and $k_{t}=/ /_{t}^{f}(\gamma)$ be parallel translation along $\sigma$ and $\gamma$ respectively so that (in both scenarios)

$$
g_{t}:=/ /^{f}\left(\partial Q_{t}\right)=k_{t}^{-1} h_{t} .
$$

Further let (with $b_{t}$ as in Eq. (5.1))

$$
B_{t}:=\int_{0}^{t} \operatorname{Ad}_{h_{\tau}^{-1}} \delta b_{\tau} \text { and } \mathbb{B}_{t}:=\int_{0 \leq r \leq s \leq t} d B_{r} \otimes d B_{s}=\int_{0}^{t} B_{s} \otimes d B_{s}
$$

and using,

we note that

$$
\left[d \operatorname{Ad}_{h_{\tau}^{-1}}\right] d b_{\tau}=\operatorname{Ad}_{h_{\tau}^{-1}} \operatorname{ad}_{d M_{\tau}^{f}(\sigma)}\left[d M_{\tau}^{f}(\gamma)-d M_{\tau}^{f}(\sigma)\right]=0
$$

$$
B_{t}:=\int_{0}^{t} \operatorname{Ad}_{h_{\tau}^{-1}} d b_{\tau} \text { and }\left[d B_{t}\right]^{2}=\sum_{\xi \in \beta}\left[\operatorname{Ad}_{h_{\tau}^{-1}} \xi\right]^{2} d a_{t}=\kappa d a_{t},
$$

where $\left[d B_{t}\right]^{2}$ is used to denote the differential of the quadratic variation matrix of $B_{(\cdot)}$. As we have mentioned before, $\delta k_{t}^{-1}=k_{t}^{-1} \delta M^{f}(\gamma)$, and thus

$$
\delta g_{t}=k_{t}^{-1} \delta M_{t}^{f}(\gamma) h_{t}-k_{t}^{-1} \delta M_{t}^{f}(\sigma) h_{t}=k_{t}^{-1} \delta b_{t} h_{t}=g_{t} \operatorname{Ad}_{h_{t}^{-1}} \delta b_{t}=g_{t} \delta B_{t} .
$$

The integral form of Eq. (5.8) expressed in Itô's form is now given by

$$
\begin{aligned}
g_{t} & =I+\int_{0}^{t} g_{\tau} \delta B_{\tau}=I+\int_{0}^{t} g_{\tau} d B_{\tau}+\frac{1}{2} \int_{0}^{t} g_{\tau}\left[d B_{\tau}\right]^{2} \\
& =I+\int_{0}^{t} g_{\tau} d B_{\tau}+\frac{1}{2} \int_{0}^{t} g_{\tau} \kappa d a_{\tau} .
\end{aligned}
$$

Making use of Eq. (5.2) (with $\beta_{t}=B_{t}$ ) it follows that

$$
\begin{aligned}
\left\|g_{t}-I\right\|_{p} & \leq\left\|\int_{0}^{t} g_{\tau} d B_{\tau}\right\|_{p}+\frac{1}{2}\left\|\int_{0}^{t} g_{\tau} \kappa d a_{\tau}\right\|_{p} \\
& \lesssim\left[\sqrt{a_{t}}+a_{t}\right]=O_{p}\left(\sqrt{a_{t}}\right) .
\end{aligned}
$$

Feeding the expansion for $g_{(\cdot)}$ in Eq. $(5.9)$ back into the right side of Eq. $(5.9)$ gives

$$
\begin{aligned}
g_{t} & =I+\int_{0}^{t}\left[I+\int_{0}^{\tau} g_{s} d B_{s}+\frac{1}{2} \int_{0}^{\tau} g_{s} \kappa d a_{s}\right] d B_{\tau} \\
& +\frac{1}{2} \int_{0}^{t}\left[I+\int_{0}^{\tau} g_{s} d B_{s}+\frac{1}{2} \int_{0}^{\tau} g_{s} \kappa d a_{s}\right] \kappa d a_{\tau} .
\end{aligned}
$$


This identity may be rewritten as

$$
g_{t}=I+B_{t}+\frac{1}{2} \kappa a_{t}+\mathcal{R}_{t}^{\prime}+R_{t}
$$

where

$$
\begin{aligned}
\mathcal{R}_{t}^{\prime} & =\int_{0}^{t}\left[\int_{0}^{\tau} g_{s} d B_{s}\right] d B_{\tau} \text { and } \\
R_{t} & =\frac{1}{2} \int_{0}^{t}\left[\int_{0}^{\tau} g_{s} \kappa d a_{s}\right] d B_{\tau}+\int_{0}^{t}\left[\int_{0}^{\tau} g_{s} d B_{s}+\frac{1}{2} \int_{0}^{\tau} g_{s} \kappa d a_{s}\right] \kappa d a_{\tau} .
\end{aligned}
$$

Using basic estimates along with Eq. (5.2) one easily shows

$$
\begin{aligned}
\left\|R_{t}\right\|_{p} & \lesssim a_{t}^{3 / 2}+a_{t}^{3 / 2}+a_{t}^{2}=O_{p}\left(a_{t}^{3 / 2}\right) \\
\mathbb{E} \mathcal{R}_{t}^{\prime} & =0, \text { and }\left\|\mathcal{R}_{t}^{\prime}\right\|_{p} \lesssim \sqrt{\int_{0}^{T}\left\|\int_{0}^{\tau} g_{s} d B_{s}\right\|_{p}^{2} d a_{\tau}}=\sqrt{\int_{0}^{T} a_{\tau} d a_{\tau}}=\frac{a_{\tau}}{\sqrt{2}} .
\end{aligned}
$$

Similarly

$$
B_{t}=\int_{0}^{t} \operatorname{Ad}_{h_{\tau}^{-1}} d b_{\tau}=\int_{0}^{t}\left[I+\int_{0}^{\tau} \operatorname{Ad}_{h_{\tau}^{-1}} \operatorname{ad}_{\delta M_{\tau}^{f}(\sigma)}\right] d b_{\tau}=b_{t}+\mathcal{R}_{t}^{\prime \prime}
$$

where

$$
\mathcal{R}_{t}^{\prime \prime}:=\int_{0}^{t} \int_{0}^{\tau} \operatorname{Ad}_{h_{\tau}^{-1}} \operatorname{ad}_{\delta M_{\tau}^{f}(\sigma)} d b_{\tau}
$$

satisfies

$$
\mathbb{E} \mathcal{R}_{t}^{\prime \prime}=0 \text { and } \mathcal{R}_{t}^{\prime \prime}=O\left(\sqrt{a_{t}^{\sigma} \cdot a_{t}}\right)
$$

Thus we have shown

$$
g_{t}=I+b_{t}+\frac{1}{2} \kappa a_{t}+\mathcal{R}_{t}+R_{t}
$$

where $R_{t}=O\left(a_{t}^{3 / 2}\right), \mathcal{R}_{t}=\mathcal{R}_{t}^{\prime}+\mathcal{R}_{t}^{\prime \prime}$, and

$$
\left\|\mathcal{R}_{t}\right\|_{p} \leq\left\|\mathcal{R}_{t}^{\prime}\right\|_{p}+\left\|\mathcal{R}_{t}^{\prime \prime}\right\|_{p}=O_{p}\left(a_{t}\right)+O\left(\sqrt{a_{t}^{\sigma} \cdot a_{t}}\right)=O\left(\sqrt{a_{t}^{\gamma} a_{t}}\right) .
$$

This completes the proof since $b_{t}=-\hat{f}\left(Q_{t}\right)$.

Let $\tilde{K} \subset \mathbb{C}^{D \times D}$ be defined by,

$$
\tilde{K}:=\{I+s(k-I): 0 \leq s \leq 1 \text { and } k \in K\},
$$

and note that $K \subset \tilde{K}$ and $\tilde{K}$ is compact in $\mathbb{C}^{D \times D}$.

Proposition 5.5. We continue the setup in Theorem 5.4 and further suppose that $\psi: \mathbb{C}^{D \times D} \rightarrow \mathbb{C}$ is a random function taking values in $C^{3}\left(\mathbb{C}^{D \times D}, \mathbb{C}\right)$. If there exists a (non-random) constant, $C<\infty$, such that

$$
\left|D^{j} \psi(k)\right| \leq C \forall k \in \tilde{K}, \text { and } 0 \leq j \leq 3,
$$

then

(5.11) $\psi\left(g_{t}\right)=\psi(I)-\left(\nabla_{\hat{f}\left(Q_{t}\right)} \psi\right)(I)+a_{t} \frac{1}{2}\left(\Delta_{K} \psi\right)(I)+\psi^{\prime}(I) \mathcal{R}_{t}+\frac{1}{2} \psi^{\prime \prime}(I) \int_{0}^{t} b_{s} \vee d b_{s}+O\left(a_{t}^{3 / 2}\right)$, 
where, for $a, b \in \mathfrak{k} \subset \mathbb{C}^{D \times D}, a \vee b:=a \otimes b+b \otimes a$ is the symmetrization of $a \otimes b$.

Proof. Let $g_{t}:=/ / f\left(\partial Q_{t}\right)$ and $b_{t}=-\hat{f}\left(Q_{t}\right)$ (as above) and define

$$
\delta_{t}:=g_{t}-I=b_{t}+\frac{1}{2} \kappa a_{t}+\mathcal{R}_{t}+R_{t}
$$

where $\mathcal{R}_{t}$ and $R_{t}$ are as in Theorem 5.4. Notice that $I+s \delta_{t} \in \tilde{K}$ for all $t \geq 0$ and $0 \leq s \leq 1$.

By Taylor's theorem with integral remainder we have

$$
\psi\left(g_{t}\right)=\psi(I)+\psi^{\prime}(I) \delta_{t}+\int_{0}^{1} \psi^{\prime \prime}\left(I+s \delta_{t}\right)\left[\delta_{t} \otimes \delta_{t}\right](1-s) d s .
$$

Since $\psi^{(3)}$ is bounded on $\tilde{K}$,

$$
\left|\psi^{\prime \prime}\left(I+s \delta_{t}\right)-\psi^{\prime \prime}(I)\right| \lesssim\left|\delta_{t}\right|
$$

which along with the estimate in Eq. (5.10) and Hölder's inequality shows

$$
\left\|\int_{0}^{1}\left(\psi^{\prime \prime}\left(I+s \delta_{t}\right)-\psi^{\prime \prime}(I)\right)\left[\delta_{t} \otimes \delta_{t}\right](1-s) d s\right\|_{p} \lesssim\left\|\left|\delta_{t}\right|^{3}\right\|_{p}=O\left(a_{t}^{3 / 2}\right) .
$$

Combining these estimates with Eq. (5.12) implies

$$
\psi\left(g_{t}\right)=\psi(I)+\psi^{\prime}(I) \delta_{t}+\frac{1}{2} \psi^{\prime \prime}(I)\left[\delta_{t} \otimes \delta_{t}\right]+O\left(a_{t}^{3 / 2}\right),
$$

Using $R_{t}=O\left(a_{t}^{3 / 2}\right)$ and $\delta_{t} \otimes \delta_{t}-b_{t} \otimes b_{t}=O\left(a_{t}^{3 / 2}\right)$ in Eq. 5.13 then shows

$$
\begin{aligned}
\psi\left(g_{t}\right) & =\psi(I)+\psi^{\prime}(I)\left[b_{t}+\frac{1}{2} \kappa a_{t}+\mathcal{R}_{t}\right]+\frac{1}{2} \psi^{\prime \prime}(I)\left[b_{t} \otimes b_{t}\right]+O\left(a_{t}^{3 / 2}\right) \\
& =\psi(I)-\left(\nabla_{\hat{f}\left(Q_{t}\right)} \psi\right)+\frac{1}{2}\left[a_{t} \psi^{\prime}(I) \kappa+\psi^{\prime \prime}(I)\left[b_{t} \otimes b_{t}\right]\right]+\psi^{\prime}(I) \mathcal{R}_{t}+O\left(a_{t}^{3 / 2}\right) .
\end{aligned}
$$

By Itô's formula,

$$
b_{t} \otimes b_{t}=\int_{0}^{t} b_{s} \vee d b_{s}+a_{t} \cdot \sum_{\xi \in \beta} \xi \otimes \xi
$$

and so by Lemma 5.2 .

$$
a_{t} \psi^{\prime}(I) \kappa+\psi^{\prime \prime}(I)\left[b_{t} \otimes b_{t}\right]=a_{t}\left(\Delta_{K} \psi\right)(I)+\psi^{\prime \prime}(I) \int_{0}^{t} b_{s} \vee d b_{s}
$$

Combining these identities and estimates gives Eq. (5.11).

Lemma 5.6. If $u$ is a $C^{2}$-function defined in a neighborhood of $I \in K$ and $\tilde{\psi}(k):=\psi\left(k^{-1}\right)$, then $\nabla \tilde{\psi}(I)=-\nabla \psi(I)$ and $\left(\Delta_{K} \tilde{\psi}\right)(I)=\left(\Delta_{K} \psi\right)(I)$.

Proof. The elementary proof is left to the reader.

Notation 5.7. Let $\mathcal{R}_{\varepsilon}^{ \pm}=\mathcal{R}_{\varepsilon}$ and $b_{t}^{ \pm}=b_{t}$ be as in Theorem 5.4 and Eq. (5.1) respectively when $Q_{t}$ is in the upper/lower half plane. [In what follows the lower half plane region will be $R Q_{t}$ where $Q_{t}$ is the region in the upper half plane.] 
Corollary 5.8. Let $\mathbb{G}, \varepsilon>0, \mathbb{G}_{\varepsilon, \pm}$, and $U: K^{\mathbb{G}} \rightarrow \mathbb{C}$ be as in Theorem 2.27 and further assume that $U$ has been extended (arbitrarily) to a smooth function on $\left[\mathbb{C}^{D \times D}\right]^{\mathbb{G}}$. Then

$$
U\left(/ /^{f}\left(\mathbb{G}_{+, \varepsilon}\right)\right)-U\left(/^{f}\left(\mathbb{G}_{-, \varepsilon}\right)\right)=-\left(\nabla_{f\left(Q_{\varepsilon}\right)}^{e_{2}} U+\nabla_{f\left(R Q_{\varepsilon}\right)}^{e_{4}} U\right)(/ / f(\mathbb{G}))+E_{\varepsilon}^{+}-E_{\varepsilon}^{-}+O\left(a_{\varepsilon}^{3 / 2}\right)
$$

where

$$
\begin{aligned}
& E_{\varepsilon}^{+}:=\left(D^{e_{2}} U\right)(/ / f(\mathbb{G})) \mathcal{R}_{\varepsilon}^{+}+\frac{1}{2}\left(D^{e_{2}} D^{e_{2}} U\right)(/ / f(\mathbb{G})) \int_{0}^{\varepsilon} b_{s}^{+} \vee d b_{s}^{+} \text {and } \\
& E_{\varepsilon}^{-}:=\left(D^{e_{4}} U\right)\left(/ /^{f}(\mathbb{G})\right) \mathcal{R}_{\varepsilon}^{-}+\frac{1}{2}\left(D^{e_{4}} D^{e_{4}} U\right)\left(/ /^{f}(\mathbb{G})\right) \int_{0}^{\varepsilon} b_{s}^{-} \vee d b_{s}^{-} .
\end{aligned}
$$

Proof. Let $\mathbb{G}^{\prime}:=\mathbb{G} \backslash\left\{e_{2}, e_{3}\right\}$ and let us write $U(\omega)$ as $U\left(\omega\left(e_{2}\right), \omega\left(e_{4}\right), \omega\left(\mathbb{G}^{\prime}\right)\right)$. We then have,

$$
U\left(/ /^{f}\left(\mathbb{G}_{+, \varepsilon}\right)\right)=U\left(/ /^{f}\left(\partial Q_{\varepsilon}\right), I, / / f\left(\mathbb{G}^{\prime}\right)\right)
$$

and by Eq. 5.11) with $\psi(k):=U\left(k, I, / / f\left(\mathbb{G}^{\prime}\right)\right)$ it follows that

$U\left(/ /^{f}\left(\mathbb{G}_{+, \varepsilon}\right)\right)=U\left(/ /^{f}(\mathbb{G})\right)-\left(\nabla_{\hat{f}\left(Q_{\varepsilon}\right)}^{e_{2}} U\right)(/ / f(\mathbb{G}))+a_{\varepsilon} \frac{1}{2}\left(\Delta_{K}^{e_{2}} U\right)(/ / f(\mathbb{G}))+E_{\varepsilon}^{+}+O\left(a_{\varepsilon}^{3 / 2}\right)$.

Similarly

$$
U\left(/ / f\left(\mathbb{G}_{-, \varepsilon}\right)\right)=U\left(I, / / f\left(\partial R Q_{\varepsilon}\right), / /^{f}\left(\mathbb{G}^{\prime}\right)\right)
$$

and by Eq. 5.11 with $\psi(k):=U\left(I, k, / / f\left(\mathbb{G}^{\prime}\right)\right)$ it follows that

$U\left(/ / f\left(\mathbb{G}_{-, \varepsilon}\right)\right)=U\left(/ /^{f}(\mathbb{G})\right)-\left(\nabla_{\hat{f}\left(R Q_{\varepsilon}\right)}^{e_{4}} U\right)\left(/ /^{f}(\mathbb{G})\right)+a_{\varepsilon} \frac{1}{2}\left(\Delta_{K}^{e_{4}} U\right)(/ / f(\mathbb{G}))+E_{\varepsilon}^{-}+O\left(a_{\varepsilon}^{3 / 2}\right)$.

Since $U$ has extended gauge invariance at 0, Lemma 5.6 implies $\left(\Delta_{K}^{e_{4}} U\right)(/ / f(\mathbb{G}))=\left(\Delta_{K}^{e_{2}} U\right)(/ / f(\mathbb{G}))$. Using the previous identities and the fact that $\hat{f}\left(R Q_{\varepsilon}\right)=-f\left(R Q_{\varepsilon}\right)$, we may subtract Eqs. (5.18) from Eq. (5.17) to arrive at Eq. (5.14).

In order to complete the proof of Theorem 2.27 it remains to estimate the error terms, $E_{\varepsilon}^{ \pm}$, in Eqs. (5.15) and (5.16) which we will do with the aid of Lemma 5.11. Lemma 5.10 below contains the key estimate needed to make this scheme work.

Notation 5.9. For $t>0$, let $J_{t}:=[0, t] \times \mathbb{R}$ and

$$
\mathcal{B}_{t}:=\sigma\left\{f(R): R \in \mathcal{B}_{\mathbb{R}^{2}}^{o} \text { with } R \subset J_{t}\right\}
$$

be the $\sigma$-algebra generated by the white noise over $J_{t}$.

In the following lemma, let $\hat{\nabla}^{\sigma}$ be the "right" analogue of $\nabla^{\sigma}$, that is replace $\omega(b) e^{t \delta_{\sigma, b} \xi}$ by $e^{t \delta_{\sigma, b} \xi_{\omega}(b)}$ in the definition of $\nabla^{\sigma}$ in Definition 2.16 .

Lemma 5.10. Let $\varepsilon>0, \Lambda$ be a finite collection of horizontal curves over $[0, \varepsilon]$, and $V: K^{\Lambda} \rightarrow \mathbb{C}$ is a random function independent of $\mathcal{B}_{\varepsilon}$ such that $V$ takes values in $C^{2}\left(K^{\Lambda}, \mathbb{C}\right)$. If there exists a (non-random) constant, $C<\infty$, such that

$$
\sup _{|\xi|_{\mathfrak{k}}=1 \&|\eta|_{\mathfrak{k}}=1}\left[|V|+\sum_{\sigma \in \Lambda}\left|\hat{\nabla}_{\xi}^{\sigma} V\right|+\sum_{\sigma, \tau \in \Lambda}\left|\hat{\nabla}_{\xi}^{\sigma} \hat{\nabla}_{\eta}^{\tau} V\right|\right] \leq C \text { on } K^{\Lambda},
$$


then

$$
\|V(/ / f(\Lambda))-V(\mathbf{I})\|_{2}=O(\sqrt{\varepsilon})
$$

where $\mathbf{I}$ is the identity in $K^{\Lambda}$ and $/ / f(\Lambda):=\{/ / f(\sigma): \sigma \in \Lambda\} \in K^{\Lambda}$ as in Notation 2.9.

Proof. For $0 \leq t \leq \varepsilon$, let $G_{t}:=/ /_{t}^{f}(\Lambda) \in K^{\Lambda}$ and $M_{t}$ be the $L\left(\mathfrak{k}^{\Lambda}, \mathfrak{k}^{\Lambda}\right)$-valued martingale which is block diagonal having $M_{t}^{f}(\sigma)$ in the $\sigma-\sigma$ block for each $\sigma \in \Lambda$. With this notation $G_{t}$ solves the Stratonovich differential equation,

$$
\delta G_{t}=-\delta M_{t} G_{t} \text { with } G_{0}=\mathbf{I} .
$$

Although $V$ is a random function, because it is independent of $\mathcal{B}_{\varepsilon}$, we may still use the adapted stochastic calculus to find,

$$
\begin{aligned}
& V\left(/ /{ }_{\varepsilon}^{f}(\Lambda)\right)-V(\mathbf{I})=V\left(G_{\varepsilon}\right)-V\left(G_{0}\right)=-\sum_{\sigma \in \Lambda} \int_{0}^{\varepsilon}\left(\hat{\nabla}_{\delta M_{t}^{f}(\sigma)}^{\sigma} V\right)\left(G_{t}\right) \\
& (5.22) \quad=-\sum_{\sigma \in \Lambda} \int_{0}^{\varepsilon}\left(\hat{\nabla}^{\sigma} V\right)\left(G_{t}\right) d M_{t}^{f}(\sigma)+\frac{1}{2} \sum_{\sigma, \tau \in \Lambda} \int_{0}^{\varepsilon}\left(\hat{\nabla}^{\tau} \hat{\nabla}^{\sigma} V\right)\left(G_{t}\right)\left[d M_{t}^{f}(\tau) \otimes d M_{t}^{f}(\sigma)\right] .
\end{aligned}
$$

Since $\Lambda$ is a finite set, the quadratic covariances of $M_{t}^{f}(\tau)$ and $M_{t}^{f}(\sigma)$ for all $\sigma, \tau \in \Lambda$ are controlled by $d t$ and therefore,

$$
\left|\sum_{\sigma, \tau \in \Lambda} \int_{0}^{\varepsilon}\left(\hat{\nabla}^{\tau} \hat{\nabla}^{\sigma} V\right)\left(G_{t}\right)\left[d M_{t}^{f}(\tau) \otimes d M_{t}^{f}(\sigma)\right]\right| \leq C_{1} \varepsilon
$$

and (from the estimate in Eq. (5.2)

$$
\left\|\sum_{\sigma \in \Lambda} \int_{0}^{\varepsilon}\left(\hat{\nabla}^{\sigma} V\right)\left(G_{t}\right) d M_{t}^{f}(\sigma)\right\|_{2} \leq C_{2} \sqrt{\varepsilon} .
$$

These estimates along with Eq. (5.22) prove Eq. (5.21).

5.1. Proof of Theorem 2.27. We start by recording the following elementary covariance estimate.

Lemma 5.11. If $Z, \bar{Z}$, and $N$ are square integrable random variables such that $\mathbb{E} N=0$ and $\bar{Z}$ is independent of $N$, then

$$
|\mathbb{E}[N Z]|=|\mathbb{E}[N(Z-\bar{Z})]| \leq\|N\|_{2} \cdot\|Z-\bar{Z}\|_{2}
$$

As in the statement of Theorem 2.27 we will assume there is a fixed constant, $C$, independent of $\varepsilon>0$ such that $\left|S_{\varepsilon}\right| \leq C\left|Q_{\varepsilon}\right|$ for all $\varepsilon>0$ where $S_{\varepsilon}$ is the "shadow" region as in Figure 8 . This means in practice that $a_{t}^{\gamma} \leq C a_{t}$ and so the error, $\mathcal{R}_{t}=O_{p}\left(\sqrt{a_{t}^{\gamma} a_{t}}\right)$, in Eq. 5.5 may be rewritten as $\mathcal{R}_{t}=O_{p}\left(a_{t}\right)$. We are now in a position to give a rigorous proof of Theorem 2.27.

Proof of Theorem 2.27. Using $a_{\varepsilon} \leq c \varepsilon$ for some $c>0$, according to Corollary 5.8 to finish the proof of Theorem 2.27 it suffices to show $\left|\mathbb{E}\left[E_{\varepsilon}^{+}-E_{\varepsilon}^{-}\right]\right|=O\left(\sqrt{\varepsilon} a_{\varepsilon}\right)$. The error term, $E_{\varepsilon}^{+}-E_{\varepsilon}^{-}$, is a sum of four terms all of the form, $\mathcal{U}(/ / f(\mathbb{G})) W_{\varepsilon}$, where $\mathcal{U} \in C^{2}\left(K^{\mathbb{G}}, Y^{*}\right)$ with $Y=\mathfrak{k}$ or $\mathfrak{k} \otimes \mathfrak{k}$ and $W_{\varepsilon}$ is a $\mathcal{B}_{\varepsilon}$-measurable $Y$-valued random vector such that; $\mathbb{E} W_{\varepsilon}=0$ and $\left\|W_{\varepsilon}\right\|_{2}=O\left(a_{\varepsilon}\right)$. 
[Here we use $Y^{*}$ to denote the real linear functionals on $Y$.] So to finish the proof it suffices to show that the expectation of any such expression, $\mathcal{U}\left(/ /^{f}(\mathbb{G})\right) W_{\varepsilon}$, is $O\left(\sqrt{\varepsilon} a_{\varepsilon}\right)$. Before going into the details, let us give a sketch of the proof.

Let $f_{\varepsilon}:=1_{\mathbb{R}^{2} \backslash J_{\varepsilon}} f$ where $f$ is the white noise. Then $\mathcal{U}\left(/ / f_{\varepsilon}(\mathbb{G})\right)$ now depends only on the white noise over $\mathbb{R}^{2} \backslash J_{\varepsilon}$ and is therefore independent of $\mathcal{B}_{\varepsilon}$. So by Lemma 5.11 with $N=W_{\varepsilon}$, $Z=\mathcal{U}(/ / f(\mathbb{G}))$, and $\bar{Z}=\mathcal{U}\left(/ / f_{\varepsilon}(\mathbb{G})\right)$, it follows that

$$
\begin{aligned}
\left|\mathbb{E}\left[\mathcal{U}\left(/ /^{f}(\mathbb{G})\right) W_{\varepsilon}\right]\right| & \leq\left\|\mathcal{U}\left(/ /^{f}(\mathbb{G})\right)-\mathcal{U}\left(/ / f_{\varepsilon}(\mathbb{G})\right)\right\|_{2}\left\|W_{\varepsilon}\right\|_{2} \\
& \leq\left\|\mathcal{U}\left(/ /^{f}(\mathbb{G})\right)-\mathcal{U}\left(/ / f_{\varepsilon}(\mathbb{G})\right)\right\|_{2} O\left(a_{\varepsilon}\right) .
\end{aligned}
$$

The proof will be completed by showing, with the aid of Lemma 5.10, that

$$
\left\|\mathcal{U}(/ / f(\mathbb{G}))-\mathcal{U}\left(/ / f_{\varepsilon}(\mathbb{G})\right)\right\|_{2} \preceq \sqrt{\varepsilon} .
$$

We now proceed to the details.

By subdividing the paths in $\mathbb{G}$ and changing the arbitrary orientations if necessary, we may assume that all paths in $\mathbb{G}$ are either purely vertical paths or are horizontal paths oriented from left to right of the form $\left[a_{\sigma}, b_{\sigma}\right] \ni x \rightarrow \sigma(x)=(x, y(x)) \in \mathbb{R}^{2}$ with $y$ being a continuous function of $x$. Let $\mathbb{G}_{h}$ denote the horizontal paths in $\mathbb{G}$ and recall for $\sigma \in \mathbb{G}_{h}$ that $\tilde{\sigma}=\sigma\left(\left[a_{\sigma}, b_{\sigma}\right]\right)$ is the image of $\sigma$ in $\mathbb{R}^{2}$. We now define $\Lambda \subset \mathbb{G}_{h}$ to be the those paths in $\mathbb{G}_{h}$ which "cross" the $y$-axis, i.e.

$$
\Lambda=\left\{\sigma \in \mathbb{G}_{h}: \tilde{\sigma} \cap J_{\varepsilon} \neq \emptyset \text { for all } \varepsilon>0\right\} .
$$

As $\Lambda$ is a finite set there exists $\varepsilon_{0}>0$ such that $\tilde{\sigma} \cap J_{\varepsilon_{0}} \neq \emptyset$ for all $\sigma \in \Lambda$ and $\tilde{\sigma} \cap J_{\varepsilon_{0}}=\emptyset$ for all $\sigma \notin \mathbb{G}_{h} \backslash \Lambda$. We now assume that $0<\varepsilon<\varepsilon_{0}$ for the rest of the argument.

For those $\sigma \in \Lambda$ we split $\sigma$ into three paths, $\left.\sigma\right|_{\left[a_{\sigma}, 0\right]},\left.\sigma\right|_{[0, \varepsilon]}$, and $\left.\sigma\right|_{\left[\varepsilon, b_{\sigma}\right]}$ and note that

$$
/ / f(\sigma)=/ /^{f}\left(\left.\sigma\right|_{\left[\varepsilon, b_{\sigma}\right]}\right) / /^{f}\left(\left.\sigma\right|_{[0, \varepsilon]}\right) / /^{f}\left(\left.\sigma\right|_{\left[a_{\sigma}, 0\right]}\right)
$$

while

$$
/ / f_{\varepsilon}(\sigma)=/ / f\left(\left.\sigma\right|_{\left[\varepsilon, b_{\sigma}\right]}\right) / / f\left(\left.\sigma\right|_{\left[a_{\sigma}, 0\right]}\right)
$$

Define the random function, $V: K^{\Lambda} \rightarrow Y^{*}$, by

$$
V(\omega):=\mathcal{U}\left(\{/ / f(\sigma)\}_{\sigma \notin \Lambda},\left\{/ /^{f}\left(\left.\sigma\right|_{\left[\varepsilon, b_{\sigma}\right]}\right) \omega(\sigma) / / f\left(\left.\sigma\right|_{\left[a_{\sigma}, 0\right]}\right)\right\}_{\sigma \in \Lambda}\right) \forall \omega \in K^{\Lambda} .
$$

As $\mathcal{U}$ is $C^{2}, V$ is also $C^{2}$ and furthermore $V$ depends only on the white noise over $\mathbb{R}^{2} \backslash J_{\varepsilon}$ and hence is independent of $\mathcal{B}_{\varepsilon}$. For $\sigma, \tau \in \Lambda$ and $\xi, \eta \in \mathfrak{k}$ we have, with

$$
\mathbf{G}:=\left(\left\{/ /^{f}(\sigma)\right\}_{\sigma \notin \Lambda},\left\{/ /^{f}\left(\left.\sigma\right|_{\left[\varepsilon, b_{\sigma}\right]}\right) \omega(\sigma) / / f\left(\left.\sigma\right|_{\left[a_{\sigma}, 0\right]}\right)\right\}_{\sigma \in \Lambda}\right)
$$

that

and similarly

$$
\left(\hat{\nabla}_{\xi}^{\sigma} V\right)(\omega)=\left(\hat{\nabla}_{\operatorname{Ad}_{/ / f}^{\sigma}\left(\left.\sigma\right|_{\left[\varepsilon, b_{\sigma}\right]}\right)}^{\xi^{\mathcal{U}}}\right)
$$

$$
\left(\hat{\nabla}_{\eta}^{\tau} \hat{\nabla}_{\xi}^{\sigma} V\right)(\omega)=\left(\hat{\nabla}_{\mathrm{Ad}_{/ / f}^{\tau}\left(\left.\sigma\right|_{\left[\varepsilon, b_{\sigma}\right]}\right)} \eta^{\hat{\nabla}_{\mathrm{Ad} / / f}^{\sigma}\left(\left.\sigma\right|_{\left[\varepsilon, b_{\sigma}\right]}\right)}{ }^{\xi} \mathcal{U}\right)(\mathbf{G})
$$


Since the inner product on $\mathfrak{k}$ is $\operatorname{Ad}_{K}$-invariant and $\mathcal{U}$ is a $C^{2}$-function on a compact set, it follows that $V$ satisfies the estimates in Eq. 5.20). Applying Lemma 5.10 then shows

$$
\left\|\mathcal{U}(/ / f(\mathbb{G}))-\mathcal{U}\left(/ f^{f_{\varepsilon}}(\mathbb{G})\right)\right\|_{2}=\|V(/ / f(\Lambda))-V(\mathbf{I})\|_{2}=O(\sqrt{\varepsilon}) .
$$

\section{Appendix A. Appendix: connections, parallel translation, and curvature}

In this first appendix, we review a few basic facts about covariant derivatives, parallel translation, and curvature. Recall that we have assumed that our compact Lie group is a matrix Lie sub-group of $G L\left(\mathbb{C}^{D}\right) \subset \mathbb{C}^{D \times D}$ for some $D \in \mathbb{N}$.

A.1. Transformation properties. The next result recalls how $g \in \mathcal{G}$ acts on covariant differentiation, parallel translation, and curvature.

Theorem A.1 (Gauge transformed quantities). If $A \in \mathcal{A}, g \in \mathcal{G}, \ell:[a, b] \rightarrow M$ is an absolutely continuous path in $M$, and $S:[a, b] \rightarrow \mathbb{C}^{D}$ (or $S:[a, b] \rightarrow \mathbb{C}^{D \times D}$ ) be a $C^{1}$-function, then

(1) The operator $\nabla_{t}^{A^{g}}$ is conjugate to $\nabla_{t}^{A}$. More precisely,

$$
\nabla_{t}^{A^{g}} S(t)=g(\ell(t))^{-1} \nabla_{t}^{A}[g(\ell(t)) S(t)]
$$

so that $\nabla_{t}^{A^{g}}=M_{g(\ell(t))^{-1}} \nabla_{t}^{A} M_{g(\ell(t))}$ where $M_{g}$ is used to denote multiplication by $g$.

(2) For $t \in[a, b]$,

$$
/{ }_{t}^{A^{g}}(\ell)=g(\ell(t))^{-1} /{ }_{t}^{A}(\ell) g(\ell(a)) .
$$

(3) The curvature tensor, $F^{A}$, satisfies,

$$
F^{A^{g}}\langle v, w\rangle=\operatorname{Ad}_{g(x)^{-1}} F^{A}\langle v, w\rangle \text { for all } v, w \in T_{x} M \text { and } x \in M .
$$

Proof. Equation A.1 follows by direct computation using the product rule and basic calculus. The proof of Eq. A.2 is now elementary as $u_{t}:=g(\ell(t))^{-1} / /{ }_{t}^{A}(\ell) g(\ell(a))$ satisfies,

$$
\nabla_{t}^{A^{g}} u_{t}=g(\ell(t))^{-1} \nabla_{t}^{A}\left[g(\ell(t)) g(\ell(t))^{-1} / /_{t}^{A}(\ell) g(\ell(a))\right]=0 \text { with } u_{a}=I .
$$

Although the curvature assertion in Eq. A.3 may be proved by direct calculation, let us give a more conceptual proof which makes use of the fact that curvature is related to the commutator of two covariant derivatives. More precisely, let $\Sigma(s, t) \in M$ and $S(s, t) \in \mathbb{C}^{D}$ (or $\mathbb{C}^{D \times D}$ ) be two $C^{1}$-functions of $(s, t) \in \mathbb{R}^{2}$ and let

$$
\nabla_{t}^{A}:=\frac{d}{d t}+A(\dot{\Sigma}(t, s)) \text { and } \nabla_{s}^{A}:=\frac{d}{d s}+A\left(\Sigma^{\prime}(t, s)\right) .
$$

A straightforward computation, using $\left[\frac{d}{d t}, \frac{d}{d s}\right]=0$ and Cartan's formula,

$$
\frac{d}{d t} A\left(\Sigma^{\prime}(t, s)\right)-\frac{d}{d s} A(\dot{\Sigma}(t, s))=d A\left(\dot{\Sigma}(t, s), \Sigma^{\prime}(t, s)\right)
$$

shows

$$
\left[\nabla_{t}^{A}, \nabla_{s}^{A}\right] S(s, t)=F^{A}\left(\dot{\Sigma}(t, s), \Sigma^{\prime}(t, s)\right) S(s, t)
$$


Thus it follows that

$$
\begin{aligned}
& F^{A^{g}}\left(\dot{\Sigma}(t, s), \Sigma^{\prime}(t, s)\right) S(s, t)=\left[\nabla_{t}^{A^{g}}, \nabla_{s}^{A^{g}}\right] S(s, t) \\
& =\left[M_{g(\Sigma(s, t))^{-1}} \nabla_{t}^{A} M_{g(\Sigma(s, t))}, M_{g(\Sigma(s, t))^{-1}} \nabla_{s}^{A} M_{g(\Sigma(s, t))}\right] S(s, t) \\
& =M_{g(\Sigma(s, t))^{-1}}\left[\nabla_{t}^{A}, \nabla_{s}^{A}\right] M_{g(\Sigma(s, t))} S(s, t) \\
& =M_{g(\Sigma(s, t))^{-1}} F^{A}\left(\dot{\Sigma}(t, s), \Sigma^{\prime}(t, s)\right) M_{g(\Sigma(s, t))} S(s, t)
\end{aligned}
$$

from which Eq. A.3 is easily deduced.

Remark A.2. One more formula connecting covariant differentiation to parallel translation is the identity;

$$
\nabla_{t}^{A} S=/ /_{t}^{A}(\ell) \frac{d}{d t}\left[/{ }_{t}^{A}(\ell)^{-1} S(t)\right]
$$

To prove this let $V(t):=/ /_{t}^{A}(\ell)^{-1} S(t)$ so that $S(t)=/ /_{t}^{A}(\ell) V(t)$. Now apply the product rule and use $\nabla_{t}^{A} / /_{t}^{A}(\ell)=0$ to find,

$$
\begin{aligned}
\nabla_{t}^{A} S(t) & =\nabla_{t}^{A}\left[/ /{ }_{t}^{A}(\ell) V(t)\right]=\left(\frac{d}{d t}+A(\dot{\ell}(t))\right)\left[/ /{ }_{t}^{A}(\ell) V(t)\right] \\
& =\left[\left(\frac{d}{d t}+A(\dot{\ell}(t))\right) / /{ }_{t}^{A}(\ell)\right] V(t)+/ /{ }_{t}^{A}(\ell) \dot{V}(t)=/ /{ }_{t}^{A}(\ell) \dot{V}(t)
\end{aligned}
$$

which is Eq. A.5.

Proposition A.3 (Connections and diffeomorphisms). Let $A \in \mathcal{A}, \sigma \in C^{1}([a, b], M)$, and $\varphi$ : $M \rightarrow M$ be a diffeomorphism of $M$. Then $F^{\varphi^{*} A}=\varphi^{*} F^{A}$ and $/ /_{t}^{\varphi^{*} A}(\sigma)=/{ }_{t}^{A}(\varphi \circ \sigma)$ for all $a \leq t \leq b$.

Proof. Using the basic properties of pull backs on forms we have,

$$
\varphi^{*} F^{A}=\varphi^{*}[d A+A \wedge A]=d\left[\varphi^{*} A\right]+\varphi^{*} A \wedge \varphi^{*} A=F^{\varphi^{*} A} .
$$

For the second assertion we compute,

$$
\begin{aligned}
0 & =\frac{\nabla}{d t} / /_{t}^{A}(\varphi \circ \sigma):=\left[\frac{d}{d t}+A\left\langle\frac{d}{d t}(\varphi \circ \sigma(t))\right\rangle\right] / /_{t}^{A}(\varphi \circ \sigma) \\
& =\left[\frac{d}{d t}+A\left\langle\varphi_{*} \dot{\sigma}(t)\right\rangle\right] /{ }_{t}^{A}(\varphi \circ \sigma)=\left[\frac{d}{d t}+\left(\varphi^{*} A\right)\langle\dot{\sigma}(t)\rangle\right] /{ }_{t}^{A}(\varphi \circ \sigma)
\end{aligned}
$$

from which we see that $/ /_{t}^{A}(\varphi \circ \sigma)$ satisfies the same differential equation as $/ /_{t}^{\varphi^{*} A}(\sigma)$.

Corollary A.4. If $\operatorname{dim} M=2, A \in \mathcal{A}$, and $\varphi: M \rightarrow M$ is an area preserving diffeomorphism, then $\left\|F^{\varphi^{*} A}\right\|^{2}=\left\|F^{A}\right\|^{2}$.

Proof. By definition,

$$
\left\|F^{\varphi^{*} A}\right\|^{2}=\int_{M}\left|F^{\varphi^{*} A}\right|^{2} d \operatorname{Vol}_{g}=\int_{M}\left|\varphi^{*} F^{A}\right|^{2} d \operatorname{Vol}_{g}
$$


Since $d=2$, if we let $\omega$ denote the (local) Riemannian volume form on $M$ then $F^{A}=f \cdot \omega$ for some $f: M \rightarrow \mathfrak{k}$. The assumption that $\varphi$ is area preserving means $\varphi^{*} \omega= \pm \omega$ and therefore,

$$
\varphi^{*} F^{A}=f \circ \varphi \cdot \varphi^{*} \omega= \pm f \circ \varphi \cdot \omega .
$$

As $\omega\left(e_{1}, e_{2}\right)= \pm 1$ where $\left\{e_{1}, e_{2}\right\}$ is any local orthonormal frame on $M$, we find

$$
\left|\varphi^{*} F^{A}\right|^{2}=|f \circ \varphi|^{2}=\left|F^{A}\right|^{2} \circ \varphi .
$$

Using this result in Eq. A.6) gives,

$$
\left\|F^{\varphi^{*} A}\right\|^{2}=\int_{M}\left|F^{A}\right|^{2} \circ \varphi d \operatorname{Vol}_{g}=\int_{M}\left|F^{A}\right|^{2} d \operatorname{Vol}_{g}=\left\|F^{A}\right\|^{2},
$$

where in the second equality we have the area preserving assumption again, namely that $\varphi_{*} \operatorname{Vol}_{g}=$ $\mathrm{Vol}_{g}$.

\section{A.2. Differential properties of parallel translation.}

Proposition A.5 (Connection Comparison). Suppose that $A$ and $B$ are two connection 1-forms, $\ell \in C^{1}([0,1], M)$, and $k_{t}:=/\left.\right|_{t} ^{B}(\ell) /{ }_{t}^{A}(\ell)^{-1}$, then

$$
\dot{k}_{t}+\left(\operatorname{Ad}_{/ / t_{t}^{A}(\ell)^{-1}}[B(\dot{\ell}(t))-A(\dot{\ell}(t))]\right) k_{t}=0 \text { with } k_{0}=0 .
$$

Proof. Since $/ /{ }_{t}^{B}(\ell)=/ /_{t}^{A}(\ell) k_{t}, \nabla_{t}^{B} / /_{t}^{B}(\ell)=0=\nabla_{t}^{A} / /_{t}^{A}(\ell)$ it follows that

$$
\begin{aligned}
0 & =\nabla_{t}^{B} / /_{t}^{B}(\ell)=\left(\nabla_{t}^{B} / /_{t}^{A}(\ell)\right) k_{t}+/ /_{t}^{A}(\ell) \dot{k}_{t} \\
& =\left(\left[\nabla_{t}^{B}-\nabla_{t}^{A}\right] / /{ }_{t}^{A}(\ell)\right) k_{t}+/ /_{t}^{A}(\ell) \dot{k}_{t} \\
& =[B(\dot{\ell}(t))-A(\dot{\ell}(t))] / /_{t}^{A}(\ell) k_{t}+/\left.\right|_{t} ^{A}(\ell) \dot{k}_{t}
\end{aligned}
$$

from which Eq. A.7 follows.

Proposition A.6 (Connection Differentiation). If $\eta$ is a $\mathfrak{k}$-valued one form on $M$ and $\ell \in$ $C^{1}([0,1], M)$, then

$$
\begin{aligned}
\partial_{\eta}\left[A \rightarrow / /^{A}(\ell)\right] & =-/{ }^{A}(\ell) \int_{0}^{1} \operatorname{Ad}_{/ / t_{t}^{A}(\ell)^{-1} \eta}(\dot{\ell}(t)) d t \\
& =-\left[\int_{0}^{1} \operatorname{Ad}_{/ / t^{A}(\ell)} \eta(\dot{\ell}(t)) d t\right] / /^{A}(\ell)
\end{aligned}
$$

Proof. First proof. Differentiating the identity, $0=\nabla_{t}^{A+s \eta} /\left.\right|_{t} ^{A+s \eta}(\ell)$ with respect to $s$ gives,

$$
\begin{aligned}
0 & =\left.\frac{d}{d s}\right|_{0}\left[\nabla_{t}^{A+s \eta} / /_{t}^{A+s \eta}(\ell)\right] \\
& =\left[\left.\frac{d}{d s}\right|_{0} \nabla_{t}^{A+s \eta}\right] / /{ }_{t}^{A}(\ell)+\nabla_{t}^{A}\left[\left.\frac{d}{d s}\right|_{0} /\left.\right|_{t} ^{A+s \eta}(\ell)\right] \\
& =\eta(\dot{\ell}(t)) / /_{t}^{A}(\ell)+\nabla_{t}^{A} \partial_{\eta} / /_{t}^{A}(\ell) \\
& =\eta(\dot{\ell}(t)) / /_{t}^{A}(\ell)+/ /_{t}^{A}(\ell) \frac{d}{d t}\left[/ /{ }_{t}^{A}(\ell)^{-1} \partial_{\eta} / / t^{A}(\ell)\right]
\end{aligned}
$$


wherein we have used Eq. A.5 in the last equality. Multiplying this equation by $/ /\left.\right|_{t} ^{A}(\ell)^{-1}$ and then integrating the result easily gives Eq. A.8) which is equivalent to Eq. A.9).

Second proof. Letting $B=A+s \eta$ in Proposition A.5 shows $/ /_{t}^{A+s \eta}(\ell)=/ /_{t}^{A}(\ell) k_{t}^{s}$ where

$$
\dot{k}_{t}^{s}+s\left(\operatorname{Ad}_{/ /{ }_{t}^{A}(\ell)^{-1} \eta}(\dot{\ell}(t))\right) k_{t}^{s}=0 \text { with } k_{0}^{s}=0 .
$$

Differentiating this equation with respect to $s$ at $s=0$ while using $k_{t}^{0}=I$ shows

$$
\left.\frac{d}{d s}\right|_{0} \dot{k}_{t}^{s}+\operatorname{Ad}_{/ / t_{t}^{A}(\ell)^{-1} \eta}(\dot{\ell}(t))=0
$$

and then integrating this result relative to $t$ shows

Hence it follows that

$$
\left.\frac{d}{d s}\right|_{0} k_{t}^{s}=-\int_{0}^{t} \operatorname{Ad}_{/ /{ }_{\tau}^{A}(\ell)^{-1} \eta}(\dot{\ell}(\tau)) d \tau
$$

$$
\begin{aligned}
\partial_{\eta}\left[A \rightarrow / /_{t}^{A}(\ell)\right] & =\left.\frac{d}{d s}\right|_{0} /\left.\right|_{t} ^{A+s \eta}(\ell)=\left.\frac{d}{d s}\right|_{0} / /_{t}^{A}(\ell) k_{t}^{s} \\
& =-/\left.\right|_{t} ^{A}(\ell) \int_{0}^{t} \operatorname{Ad}_{/ /{ }_{\tau}^{A}(\ell)^{-1}} \eta(\dot{\ell}(\tau)) d \tau
\end{aligned}
$$

Proposition A.7 (Path Differentiation). Suppose $\ell_{s}$ is a one parameter family of curves parametrized by an interval, $[0,1]$ such that $\ell_{s}(0)$ is constant independent of $s$ and let $/ /{ }_{t}^{A}(\ell)$ denote parallel translation along $\left.\ell_{s}\right|_{[0, t]}$. Then,

$$
\frac{\nabla^{A}}{d s} / /_{t}^{A}\left(\ell_{s}\right)=/ /_{t}^{A}\left(\ell_{s}\right) \int_{0}^{t} \operatorname{Ad}_{/ / \tau_{\tau}^{A}\left(\ell_{s}\right)^{-1}} F^{A}\left(\dot{\ell}_{s}(\tau), \ell_{s}^{\prime}(\tau)\right) d \tau
$$

and, if we further assume that $\ell_{s}(1)$ is constant independent of $s$, then

$$
\frac{d}{d s} / /_{1}^{A}\left(\ell_{s}\right)=/ /_{1}^{A}\left(\ell_{s}\right) \int_{0}^{1} \operatorname{Ad}_{/ /{ }_{\tau}^{A}\left(\ell_{s}\right)^{-1}} F^{A}\left(\dot{\ell}_{s}(\tau), \ell_{s}^{\prime}(\tau)\right) d \tau .
$$

Equation A.10 may also be expressed as,

$$
\frac{d}{d s}\left[/ /{ }_{s}^{A}\left(\ell_{(\cdot)}(t)\right)^{-1} / /{ }_{t}^{A}\left(\ell_{s}\right)\right]=\left[/ /{ }_{s}^{A}\left(\ell_{(\cdot)}(t)\right)^{-1} / /{ }_{t}^{A}\left(\ell_{s}\right)\right] \int_{0}^{t} \operatorname{Ad}_{/ / A_{\tau}^{A}\left(\ell_{s}\right)^{-1}} F^{A}\left(\dot{\ell}_{s}(\tau), \ell_{s}^{\prime}(\tau)\right) d \tau .
$$

Proof. By Eq. A.4 and the fact that $\frac{\nabla^{A}}{d t} / /{ }_{t}^{A}\left(\ell_{s}\right)=0$,

$$
\frac{\nabla^{A}}{d t} \frac{\nabla^{A}}{d s} /{ }_{t}^{A}\left(\ell_{s}\right)=\left[\frac{\nabla^{A}}{d t}, \frac{\nabla^{A}}{d s}\right] / / t_{t}^{A}\left(\ell_{s}\right)=F^{A}\left(\dot{\ell}_{s}(t), \ell_{s}^{\prime}(t)\right) / /_{t}^{A}\left(\ell_{s}\right) .
$$

By Remark A.2, the last identity may be rewritten as,

$$
\frac{d}{d t}\left[/ / t_{t}^{A}(\ell)^{-1} \frac{\nabla^{A}}{d s} / /_{t}^{A}\left(\ell_{s}\right)\right]=/\left.\right|_{t} ^{A}(\ell)^{-1} F^{A}\left(\dot{\ell}(t), \ell_{s}^{\prime}(t)\right) / /_{t}^{A}(\ell) .
$$

Integrating this equation on $t$ gives Eq. A.10). If we now assume $\ell_{s}(1)$ is constant in $s$, then

$$
\frac{\nabla^{A}}{d s} / /_{1}^{A}\left(\ell_{s}\right)=\left(\frac{d}{d s}+A\left\langle\ell_{s}^{\prime}(1)\right\rangle\right) / /_{1}^{A}\left(\ell_{s}\right)=\frac{d}{d s} / /_{1}^{A}\left(\ell_{s}\right)
$$


which combined with Eq. A.10) at $t=1$ gives Eq. A.11).

For more information on Proposition A.7 much more related material to this and the next appendix, see [18] and [12].

\section{Appendix B. Homotopy gauge Fixing of YAng-Mills}

The goal of this appendix is to motivate the definition of the Yang-Mills measure as used in this paper. We also wish to give a heuristic argument that the resulting expectations should be invariant under area preserving diffeomorphisms. We begin with a few general results in finite dimensions which we will later apply (illegally) in the infinite dimensional Yang-Mills context. For an interesting general discussion of gauge fixing from a differential form point, as apposed to the more measure theoretic view described in this appendix, see [29, Section III]. There is of course a huge physics literature on various methods of gauge fixing which we do not attempt to survey here. However, the interested reader might start with Chapter 13 in [11, Section 13.6] or Chapter 15 in [34] and then consult some of the references in [29].

B.1. Group actions and gauges. We will use the following notation throughout this subsection.

Notation B.1. Let $(\mathcal{A}, \mathcal{G}, m, \lambda)$ be a quadruple consisting of a smooth manifold, $\mathcal{A}$, a Lie group $\mathcal{G}$, a smooth measure $(m)$ on $\mathcal{A}$, and a right invariant Haar measure $(\lambda)$ on $\mathcal{G}$. We assume that there is a given right action of $\mathcal{G}$ on $\mathcal{A}$ and that the measure $m$ is invariant under this right action, i.e. $m$ is invariant under the transformation, $\mathcal{A} \ni A \rightarrow A g \in \mathcal{A}$ for each $g \in \mathcal{G}$.

Definition B.2. A gauge is a smooth function, $v: \mathcal{A} \rightarrow \mathcal{G}$, such that $v(A g)=v(A) g$ for all $A \in \mathcal{A}$ and $g \in \mathcal{G}$. Associated to $v$ we defined the "projection map," $\pi_{v}: \mathcal{A} \rightarrow \mathcal{A}$, by $\pi_{v}(A):=$ $A \cdot v(A)^{-1}$ and let

$$
\mathcal{A}_{v}:=\pi_{v}(\mathcal{A})=\left\{A \cdot v(A)^{-1}: A \in \mathcal{A}\right\} .
$$

Lemma B.3. If $v: \mathcal{A} \rightarrow \mathcal{G}$ is a gauge and $A, B \in \mathcal{A}$, then;

(1) $\pi_{v}$ is constant on gauge orbits,

(2) $\pi_{v} \circ \pi_{v}=\pi_{v}$ (i.e. $\left.\pi_{v}\right|_{\mathcal{A}_{v}}$ is the identity on $\mathcal{A}_{v}$ ),

(3) $\mathcal{A}_{v}$ may also be expresses as

$$
\mathcal{A}_{v}=\{A \in \mathcal{A}: v(A)=I \in \mathcal{G}\},
$$

(4) $\mathcal{A}_{v}$ is an embedded submanifold of $\mathcal{A}$,

(5) $\pi_{v}(A)=\pi_{v}(B)$ iff $A$ and $B$ are in the same $\mathcal{G}$-orbit, and

(6) the map,

$$
\mathcal{A}_{v} \times \mathcal{G} \ni(A, g) \rightarrow A \cdot g \in \mathcal{A}
$$

is as diffeomorphism of smooth manifolds.

Proof. We take each item in turn.

(1) If $A \in \mathcal{A}$ and $g \in \mathcal{G}$, then

$$
\pi_{v}(A g)=A g \cdot v(A g)^{-1}=A g \cdot[v(A) g]^{-1}=A \cdot v(A)^{-1}=\pi_{v}(A)
$$

which shows $\pi_{\nu}$ is constant on $\mathcal{G}$-orbits.

(2) If $A \in \mathcal{A}$, then $A$ and $\pi_{v}(A)$ are in the same gauge orbit and hence $\pi_{v}\left(\pi_{v}(A)\right)=\pi_{v}(A)$. 
(3) If $A \in \mathcal{A}_{v}$ then $A=\pi_{\nu}(A)=A \cdot v(A)^{-1}$ and therefore,

$$
v(A)=v\left(A \cdot v(A)^{-1}\right)=v(A) \cdot v(A)^{-1}=I .
$$

Conversely if $\nu(A)=I$, then $\pi_{v}(A)=A \in \mathcal{A}_{v}$.

(4) If $A \in \mathcal{A}$ and $\xi \in \operatorname{Lie}(\mathcal{G})=T_{I} \mathcal{G}$, then

$$
\left.\frac{d}{d t}\right|_{0} v\left(A e^{t \xi}\right)=\left.\frac{d}{d t}\right|_{0}\left[v(A) e^{t \xi}\right]=L_{v(A) *} \xi
$$

where the latter expression varies over $T_{v(A)} G$ as $\xi$ varies over Lie $(\mathcal{G})$. This shows $\nu$ is a submersion and so the level sets of $v$ are all embedded submanifolds, in particular $\mathcal{A}_{v}=$ $v^{-1}(\{I\})$ is an embedded submanifold.

(5) The condition that $\pi_{v}(A)=\pi_{v}(B)$ is equivalent to $A \cdot v(A)^{-1}=B \cdot v(B)^{-1}$ which is then equivalent to $B=A \cdot\left[v(A)^{-1} v(B)\right]$, i.e. $B$ and $A$ are in the same gauge orbit.

(6) The inverse to the smooth map in Eq. (B.1) is the smooth map, $\mathcal{A} \ni A \rightarrow\left(\pi_{v}(A), v(A)\right)$.

Example B.4 (Product groups). Let $\mathcal{G}$ be a Lie group, $N \in \mathbb{N}, \mathcal{A}=\mathcal{G}^{N}$, and let $\mathcal{G}$ act on $\mathcal{A}$ on the right by the diagonal action,

$$
\mathcal{A} \times \mathcal{G} \ni(\vec{g}, k) \rightarrow \vec{g} \cdot k \in \mathcal{A} \text { where }[\vec{g} \cdot k]_{i}=g_{i} k \text { for } 1 \leq i \leq N .
$$

Then $\nu: \mathcal{A} \rightarrow \mathcal{G}$ defined by $v(\vec{g})=g_{1}$ is a gauge. In this case

$$
\pi_{v}(\vec{g})=\vec{g} \cdot g_{1}^{-1}=\left(\begin{array}{c}
e \\
g_{2} g_{1}^{-1} \\
\vdots \\
g_{N} g_{1}^{-1}
\end{array}\right)
$$

and $\mathcal{A}_{v}=\{e\} \times \mathcal{G}^{N-1}$.

Example B.5. Let $\mathcal{A}=\mathbb{R}^{n}, \mathcal{G}=\mathbb{R}, \xi \in \operatorname{sl}(n, \mathbb{R})$ such that $\xi_{l k}=0$ if either $l$ or $k=n$ and for $x \in \mathbb{R}^{n}$ (thought of as row vector) and $t \in \mathbb{R}$ let

$$
x \cdot t:=x e^{t \xi}+t e_{n}=\left[x+t e_{n}\right] e^{t \xi} .
$$

Since $e_{n} \xi=0$ we have $e_{n} e^{t \xi}=e_{n}$ and by assumption $e^{t \xi}$ preserves $\operatorname{span}\left(e_{k}\right)_{k<n}$ and hence

$$
(x \cdot t) \cdot s=\left(x e^{t \xi}+t e_{n}\right) e^{s \xi}+s e_{n}=x e^{t \xi} e^{s \xi}+t e_{n}+s e_{n}=x \cdot(t+s) .
$$

In this case the projection map, $v(x)=x_{n}$ is a gauge with

$$
\pi_{\nu}(x)=x e^{-x_{n} \xi}-x_{n} e_{n}=\left(\begin{array}{c}
x_{1} \\
\vdots \\
x_{n-1} \\
0
\end{array}\right) e^{-x_{n} \xi} \text { and } \mathcal{A}_{v}=\mathbb{R}^{n-1} \times\{0\} .
$$

Example B.6. Let us specializing Example B.5 to $n=3$ and

$$
\xi=\left[\begin{array}{ccc}
0 & -1 & 0 \\
1 & 0 & 0 \\
0 & 0 & 0
\end{array}\right] \quad \Longrightarrow \quad e^{t \xi}=\left[\begin{array}{ccc}
\cos t & -\sin t & 0 \\
\sin t & \cos t & 0 \\
0 & 0 & 1
\end{array}\right]
$$


In this case the gauge orbits are spirals. For example, the gauge orbit of $e_{1}=(1,0,0) \in \mathbb{R}^{3}$ is the spiral, $\left\{e_{1} \cdot t=(\cos t,-\sin t, t): t \in \mathbb{R}\right\}$.

Examples B.5 and B.6 were examples of "affine actions," which we now define.

Definition B.7 (Affine actions). Assume $(\mathcal{A}, \mathcal{G})$ as above with $\mathcal{A}$ being a finite dimensional vector space and let $S L(\mathcal{A})$ denote the special linear transformations on $\mathcal{A}$. We say the group action of $\mathcal{G}$ on $\mathcal{A}$ is an affine action if it may be written in the form;

$$
A g=\rho\left(g^{-1}\right) A+T(g)
$$

where $\rho: \mathcal{G} \rightarrow S L(\mathcal{A})$ is a representation of $\mathcal{G}$ and $T: \mathcal{G} \rightarrow \mathcal{A}$ is a smooth function.

Remark B.8. It is left to the interested reader to verify that $T(e)=0$ and the pair, $(\rho, T)$, must satisfy the "cocylcle" condition;

$$
T(g h)=\rho\left(h^{-1}\right) T(g)+T(h) \forall g, h \in \mathcal{G} .
$$

The key formal example of an affine action is the right action of the restricted gauge group acting on connection one forms as in Eq. (1.1). We will work heuristically with this formal infinite dimensional setup in Subsection B.4 below.

\section{B.2. Disintegration formulas.}

Proposition B.9 (Disintegration). Let $(\mathcal{A}, \mathcal{G}, m, \lambda)$ be as in Notation B.1. To each gauge, $v$ : $\mathcal{A} \rightarrow \mathcal{G}$, there exists a unique (smooth) measure $m_{v}$ on $\mathcal{A}_{v}$ such that

$$
\int_{\mathcal{A}} f(A) d m(A)=\int_{\mathcal{A}_{v}} d m_{v}(B) \int_{\mathcal{G}} d \lambda(g) f(B g)
$$

for all $f: \mathcal{A} \rightarrow[0, \infty]$ measurable.

Proof. Let $\gamma$ be a fixed smooth measure on $\mathcal{A}_{v}$. Since the map in Eq. (B.1) is a diffeomorphism and Haar measure, $\lambda$, is a smooth measure on $\mathcal{G}$, there exists a smooth density, $\mu: \mathcal{A}_{v} \times \mathcal{G} \rightarrow(0, \infty)$, such that

$$
\int_{\mathcal{A}} f(A) d m(A)=\int_{\mathcal{A}_{v}} d \gamma(B) \int_{\mathcal{G}} d \lambda(g) \mu(B, g) f(B g)
$$

for all $f: \mathcal{A} \rightarrow[0, \infty]$ measurable. Using the invariance of $m$ and $\lambda$ under the right $\mathcal{G}$-actions on $\mathcal{A}$ and $\mathcal{G}$ respectively, if $k \in \mathcal{G}$, then

$$
\begin{aligned}
\int_{\mathcal{A}} f(A) d m(A) & =\int_{\mathcal{A}} f(A k) d m(A) \\
& =\int_{\mathcal{A}_{v}} d \gamma(B) \int_{\mathcal{G}} d \lambda(g) \mu(B, g) f(B g k) \\
& =\int_{\mathcal{A}_{v}} d \gamma(B) \int_{\mathcal{G}} d \lambda(g) \mu\left(B, g k^{-1}\right) f(B g) .
\end{aligned}
$$

Comparing Eqs. B.5) and B.6) implies $\mu\left(B, g k^{-1}\right)=\mu(B, g)$ for all $B \in \mathcal{A}_{v}$ and $g, k \in \mathcal{G}$. Taking $k=g$ shows $\mu(B, g)=\mu(B, e)$ and so Eq. (B.4) holds with $d m_{v}(B):=\mu(B, e) d \gamma(B)$.

Theorem B.10 (Affine Action Disintegrations). Assume $(\mathcal{A}, \mathcal{G})$ as above with $\mathcal{A}$ being a finite dimensional vector space equipped with an affine action of $\mathcal{G}$ on $\mathcal{A}$, see Definition B.7. Then; 
(1) Lebesgue measure $(m)$ on $\mathcal{A}$ is invariant under the $\mathcal{G}$-action.

(2) If $v: \mathcal{A} \rightarrow \mathcal{G}$ is a gauge such that $\mathcal{A}_{v}$ is a linear subspace which is invariant under the action of $\rho$, then the measure $\left(m_{\nu}\right)$ in Proposition B.9 is a Lebesgue measure on $\mathcal{A}_{v}$.

Proof. 1. The Jacobian-determinant factor for the change of variables, $B=A g$, is $\left|\operatorname{det} \rho\left(g^{-1}\right)\right|=$ 1 and hence the affine transformation $A \rightarrow A g$ leaves $m$ invariant on $\mathcal{A}$.

2. Let $m_{0}$ be a Lebesgue measure on $\mathcal{A}_{v}$ (i.e. a translation invariant Radon measure on $\mathcal{A}_{v}$ ). The smooth measure $\left(m_{\nu}\right)$ may be expressed as $d m_{\nu}(A)=\mu(A) d m_{0}(A)$ for some smooth density $\mu: \mathcal{A} \rightarrow(0, \infty)$. Our goal is to show that $\mu$ is a constant.

According to Proposition B.9, if $f: \mathcal{A} \rightarrow[0, \infty]$ is measurable, then

$$
\int_{\mathcal{A}} f(C) d m(C)=\int_{\mathcal{A}_{v}} d m_{\nu}(A) \int_{\mathcal{G}} d \lambda(g) f(A \cdot g)=\int_{\mathcal{G}} d \lambda(g) \int_{\mathcal{A}_{v}} d m_{0}(A) \mu(A) f(A \cdot g) .
$$

Let $B \in \mathcal{A}_{v}$ and apply Eq. (B.7) with $f$ replaced by $f(\cdot+B)$ to find

$$
\begin{aligned}
\int_{\mathcal{A}} f(C+B) d m(C) & =\int_{\mathcal{G}} d \lambda(g) \int_{\mathcal{A}_{v}} d m_{0}(A) \mu(A) f(A \cdot g+B) \\
& =\int_{\mathcal{G}} d \lambda(g) \int_{\mathcal{A}_{v}} d m_{0}(A) \mu(A) f\left(\left[A+\operatorname{Ad}_{g} B\right]^{g}\right) \\
& =\int_{\mathcal{G}} d \lambda(g) \int_{\mathcal{A}_{v}} d m_{0}(A) \mu\left(A-\operatorname{Ad}_{g} B\right) f(A \cdot g),
\end{aligned}
$$

wherein the last line we have used $m_{0}$ is a translation invariant measure. On the other hand $m$ is also translation invariant and so

$$
\int_{\mathcal{A}} f(C+B) d m(C)=\int_{\mathcal{A}} f(C) d m(C)=\int_{\mathcal{G}} d \lambda(g) \int_{\mathcal{A}_{v}} d m_{0}(A) \mu(A) f(A \cdot g) .
$$

Using the map in Eq. (B.1) is a diffeomorphism and the last two displayed equations are valid for all measurable functions, $f: \mathcal{A} \rightarrow[0, \infty]$, we conclude that $\mu\left(A-\operatorname{Ad}_{g} B\right)=\mu(A)$ for all $A, B \in \mathcal{A}_{v}$ and $g \in \mathcal{G}$. Taking $g \equiv I$ and $B=A$ then implies $\mu(A)=\mu(0)$ for all $A \in \mathcal{A}_{\nu}$, i.e. $\mu$ is constant.

B.3. Abstract gauge fixing. If $\Psi: \mathcal{A} \rightarrow[0, \infty)$ is a $\mathcal{G}$-invariant function, then from Eq. (B.4) it follows that

$$
\begin{aligned}
\int_{\mathcal{A}} \Psi(A) d m(A) & =\int_{\mathcal{A}_{v}} d m_{v}(B) \int_{\mathcal{G}} d \lambda(g) \Psi(B g) \\
& =\int_{\mathcal{A}_{v}} d m_{v}(B) \int_{\mathcal{G}} d \lambda(g) \Psi(B) \\
& =\lambda(\mathcal{G}) \cdot \int_{\mathcal{A}_{v}} \Psi(B) d m_{v}(B) .
\end{aligned}
$$

This suggests that we normalize $\int_{\mathcal{A}} \Psi(A) d m(A)$ by "dividing" the integral by $\lambda(\mathcal{G})$ and setting

$$
f_{\mathcal{A}} \Psi(A) d m(A)=" \frac{1}{\lambda(\mathcal{G})} \int_{\mathcal{A}} \Psi(A) d m(A) " .
$$

The problem with this formula is that (in the interesting cases) $\lambda(\mathcal{G})=\infty$. To avoid this division by infinity we make the following definition. 
Definition B.11. The v-normalized integral of a $\mathcal{G}$-invariant function, $\Psi: \mathcal{A} \rightarrow[0, \infty)$, is

$$
f_{\mathcal{A}} \Psi(A) d m(A)=\int_{\mathcal{A}_{v}} \Psi(B) d m_{v}(B) .
$$

Notation B.12. Let $\Delta: \mathcal{G} \rightarrow(0, \infty)$ be the modular function on $\mathcal{G}$ defined by requiring

$$
\int_{\mathcal{G}} \psi(k g) d \lambda(g)=\Delta(k) \cdot \int_{\mathcal{G}} \psi(g) d \lambda(g)
$$

for all $k \in \mathcal{G}$ and $\psi: \mathcal{G} \rightarrow[0, \infty]$ measurable. Recall $\mathcal{G}$ is said to be unimodular if $\Delta \equiv 1$.

Theorem B.13. If $\mathcal{G}$ is a unimodular Lie group, $\Psi: \mathcal{A} \rightarrow[0, \infty)$ is a $\mathcal{G}$-invariant function, and $v, w$ are two gauges, then

$$
\int_{\mathcal{A}_{v}} \Psi(B) d m_{v}(B)=\int_{\mathcal{A}_{w}} \Psi(B) d m_{w}(B) .
$$

Proof. Let $\alpha \in C(\mathcal{G},[0, \infty))$ such that $\int_{\mathcal{G}} \alpha(g) d \lambda(g)=1$ and set $f(A):=\Psi(A) \alpha(v(A))$. By Eq. (B.4) we find

$$
\begin{aligned}
\int_{\mathcal{A}} f(A) d m(A) & =\int_{\mathcal{A}_{w}} d m_{w}(B) \int_{\mathcal{G}} d \lambda(g) \Psi(B g) \alpha(v(B g)) \\
& =\int_{\mathcal{A}_{w}} d m_{w}(B) \int_{\mathcal{G}} d \lambda(g) \Psi(B) \alpha(v(B) g) \\
& =\int_{\mathcal{A}_{w}} d m_{w}(B) \int_{\mathcal{G}} d \lambda(g) \Psi(B) \Delta(v(B)) \alpha(g) \\
& =\int_{\mathcal{A}_{w}} \Psi(B) \Delta(v(B)) d m_{w}(B) .
\end{aligned}
$$

In the case $w=v$, so that $B \in \mathcal{A}_{v}$, we find

$$
\begin{aligned}
\int_{\mathcal{A}} f(A) d m(A) & =\int_{\mathcal{A}_{v}} \Psi(B) \Delta(v(B)) d m_{v}(B) \\
& =\int_{\mathcal{A}_{v}} \Psi(B) \Delta(e) d m_{v}(B)=\int_{\mathcal{A}_{v}} \Psi(B) d m_{v}(B) .
\end{aligned}
$$

Thus we have shown in general that

$$
\int_{\mathcal{A}_{v}} \Psi(B) d m_{v}(B)=\int_{\mathcal{A}_{w}} \Psi(B) \Delta(v(B)) d m_{w}(B)
$$

and in particular if $\mathcal{G}$ is unimodular, Eq. B.8 holds.

Theorem B.14. Let $(\mathcal{A}, \mathcal{G}, m, \lambda)$ be as in Notation B.1, $v: \mathcal{A} \rightarrow \mathcal{G}$ be a gauge, and $\varphi: \mathcal{A} \rightarrow \mathcal{A}$ be a diffeomorphism such that;

(1) $\varphi$ is volume preserving, i.e. $\varphi_{*} m=m$.

(2) $\varphi$ acts equivariantly on $\mathcal{A}$ in the sense that there exists a Lie group isomorphism, $\gamma: \mathcal{G} \rightarrow$ $\mathcal{G}$, such that

$$
\varphi(A g)=\varphi(A) \gamma(g) \quad \forall A \in \mathcal{A} \text { and } g \in \mathcal{G} .
$$

[Note this implies $\varphi$ preserves gauge orbits.] 
Under these assumptions, if $\Psi: \mathcal{A} \rightarrow[0, \infty]$ is a gauge invariant function, then

$$
\int_{\mathcal{A}_{v}} \Psi(\varphi(A)) d m_{v}(A)=\frac{1}{c_{\gamma}} \int_{\mathcal{A}_{v}} \Psi(A) \Delta\left(v\left(\varphi^{-1}(A)\right)\right) d m_{v}(A)
$$

where $c_{\gamma}$ is the constant determined by

$$
\gamma_{*} \lambda=c_{\gamma} \lambda
$$

Proof. For the moment let us simply suppose that $\varphi$ preserves gauge orbits which may be stated as saying $\varphi(A g)=\varphi(A) \Gamma(A, g)$ for some function $\Gamma: \mathcal{A} \times \mathcal{G} \rightarrow \mathcal{G}$. As in the proof of Theorem B.13. let $\alpha \in C(\mathcal{G},[0, \infty))$ such that $\int_{\mathcal{G}} \alpha(g) d \lambda(g)=1$ and $\Psi: \mathcal{A} \rightarrow[0, \infty]$ be a gauge invariant function in which case,

$$
\int_{\mathcal{A}_{v}} \Psi(A) d m_{v}(A)=\int_{\mathcal{A}} \Psi(A) \alpha(v(A)) d m(A) .
$$

Applying this identity with $\Psi$ replaced by $\Psi \circ \varphi$ gives,

$$
\begin{aligned}
\int_{\mathcal{A}_{v}} \Psi \circ \varphi(A) d m_{v}(A) & =\int_{\mathcal{A}} \Psi \circ \varphi(A) \alpha(v(A)) d m(A) \\
& =\int_{\mathcal{A}} \Psi(A) \alpha\left(v\left(\varphi^{-1}(A)\right)\right) d m(A) \quad\left(\varphi_{*} m=m\right) \\
& =\int_{\mathcal{A}_{v}} d m_{v}(B) \int_{\mathcal{G}} d \lambda(g) \Psi(B g) \alpha\left(v\left(\varphi^{-1}(B \cdot g)\right)\right) \\
& =\int_{\mathcal{A}_{v}} d m_{v}(B) \int_{\mathcal{G}} d \lambda(g) \Psi(B) \alpha\left(v\left(\varphi^{-1}(B \cdot g)\right)\right) \\
& =\int_{\mathcal{A}_{v}} \Psi(B) \mu_{\varphi}(B) d m_{v}(B),
\end{aligned}
$$

where $\mu_{\varphi}: \mathcal{A}_{v} \rightarrow[0, \infty)$ is defined by

$$
\mu_{\varphi}(B):=\int_{\mathcal{G}} \alpha\left(v\left(\varphi^{-1}(B \cdot g)\right)\right) d \lambda(g) .
$$

Let us now assume that $\varphi$ satisfies Eq. B.9. Applying $\varphi^{-1}$ to Eq. B.9 with $A$ replaced by $\varphi^{-1}(A)$ and $g$ by $\gamma^{-1}(g)$ implies,

$$
\varphi^{-1}(A) \gamma^{-1}(g)=\varphi^{-1}(A g) .
$$

Using this fact and noting that $\gamma_{*} \lambda=c_{\gamma} \lambda$ implies $\lambda=c_{\gamma}\left(\gamma^{-1}\right)_{*} \lambda$, if follows that

$$
\begin{aligned}
\mu_{\varphi}(B) & :=\int_{\mathcal{G}} \alpha\left(v\left(\varphi^{-1}(B) \gamma^{-1}(g)\right)\right) d \lambda(g) \\
& =\frac{1}{c_{\gamma}} \int_{\mathcal{G}} \alpha\left(v\left(\varphi^{-1}(B)\right) g\right) d \lambda(g)=c_{\gamma}^{-1} \Delta\left(v\left(\varphi^{-1}(B)\right)\right) .
\end{aligned}
$$

Combining the last equation with Eq. (B.12) gives Eq. (B.10).

Remark B.15. One might hope to relax the condition in Eq. $(B .9)$ in the previous theorem as follows. Suppose that $\varphi: \mathcal{A} \rightarrow \mathcal{A}$ is a diffeomorphism which takes gauge orbits to gauge orbits. 
Then define $\tilde{\varphi}(A g)=\pi_{v}(\varphi(A)) \cdot g$ for all $A \in \mathcal{A}_{v}$ and $g \in \mathcal{G}$. Then if $\Psi: \mathcal{A} \rightarrow[0, \infty]$ is a gauge invariant function we will have

$$
\Psi(\tilde{\varphi}(A g))=\Psi\left(\pi_{v}(\varphi(A)) \cdot g\right)=\Psi\left(\pi_{v}(\varphi(A))\right)=\Psi(\varphi(A))
$$

so that

$$
\int_{\mathcal{A}_{v}} \Psi(\varphi(A)) d m_{v}(A)=\int_{\mathcal{A}_{v}} \Psi(\tilde{\varphi}(A)) d m_{v}(A) .
$$

The point being that $\tilde{\varphi}: \mathcal{A} \rightarrow \mathcal{A}$ is a diffeomorphism such that $\tilde{\varphi}(A g k)=\pi_{v}(\varphi(A)) \cdot g k=\tilde{\varphi}(A g) k$ so that Eq. (B.9) holds with $\gamma(g)=g$. However, the problem is that there is no reason that $\tilde{\varphi}$ should still preserve $m$.

Corollary B.16. Let us continue the notation and assumptions of Theorem B.14. If we further assume that $\mathcal{G}$ is unimodular and $c_{\gamma}=1$, then

$$
f_{\mathcal{A}} \Psi(\varphi(A)) d m(A)=f_{\mathcal{A}} \Psi(A) d m(A)
$$

for all gauge invariant functions, $\Psi: \mathcal{A} \rightarrow[0, \infty]$.

Example B.17 (Example B.4 continued). Let us continue the notation in Example B.4 and further assume that $\mathcal{G}$ is a unimodular Lie group. Further let $m=\lambda^{\otimes N}$ where $\lambda$ is a Haar measure on $\mathcal{G}$ and set $v(\vec{g})=g_{1}$ so that $\mathcal{A}_{v}=\{e\} \times \mathcal{G}^{N-1}$. To make a gauge invariant function, let $f: \mathcal{G}^{N-1} \rightarrow \mathbb{C}$ be any function and the set $\Psi(\vec{g})=f\left(\left\{g_{j} g_{1}^{-1}\right\}_{j=2}^{N}\right)$. In this case, $m_{v}$ is given by $m_{v}=\delta_{e} \otimes \lambda^{\otimes(N-1)}$ since for $f: \mathcal{A} \rightarrow \mathbb{C}$ we have

$$
\begin{aligned}
\int_{\mathcal{A}} f(\vec{g}) d m(\vec{g}) & =\int_{\mathcal{A}} f\left(\begin{array}{c}
g_{1} \\
g_{2} \\
\vdots \\
g_{N}
\end{array}\right) d \lambda\left(g_{1}\right) d \lambda^{\otimes}\left(\overrightarrow{g_{\geq 2}}\right) \\
& =\int_{\mathcal{A}} f\left(\begin{array}{c}
g_{1} \\
g_{2} g_{1} \\
\vdots \\
g_{N} g_{1}
\end{array}\right) d \lambda\left(g_{1}\right) d \lambda^{\otimes}\left(\overrightarrow{g_{\geq 2}}\right) \\
& =\int_{\mathcal{A}} f\left(\begin{array}{c}
e \\
g_{2} \\
\vdots \\
g_{N}
\end{array}\right) \cdot g_{1} d \lambda\left(g_{1}\right) d \lambda^{\otimes}\left(\overrightarrow{g_{\geq 2}}\right) .
\end{aligned}
$$

For an example of $a \varphi: \mathcal{A} \rightarrow \mathcal{A}$ satisfying the assumption of Corollary B.16, fix $a, b \in \mathcal{G}$ and then define,

$$
\varphi(\vec{g})=a \cdot \vec{g} \cdot b:=\left(\begin{array}{c}
a g_{1} b \\
a g_{2} b \\
\vdots \\
a g_{N} b
\end{array}\right)
$$


Then $\varphi$ an m-preserving diffeomorphism on $\mathcal{A}$ with

$$
\varphi(\vec{g} \cdot k)=a \cdot \vec{g} \cdot k \cdot b=a \cdot \vec{g} \cdot b \cdot b^{-1} k b=\varphi(\vec{g}) \cdot \gamma(k)
$$

where $\gamma(k):=\operatorname{Ad}_{b^{-1}} k$, and so $\varphi$ satisfies the hypothesis of Corollary B.16.

B.4. Yang-Mills gauge fixing. In this section, we suppose (as defined in Notation 1.1 with $M=\mathbb{R}^{2}$ ) that $\mathcal{A}:=\Omega^{1}\left(\mathbb{R}^{2}, \mathfrak{k}\right), \mathcal{G}$ is the gauge group of functions, $g: \mathbb{R}^{2} \rightarrow K$, and $\mathcal{G}_{o}=$ $\{g \in \mathcal{G}: g(o)=I\}$ is the restricted gauge group.

Definition B.18 (Homotopies). A continuous map, $\mathbb{R}^{d} \times[0,1] \ni(x, t) \rightarrow \sigma_{x}(t) \in \mathbb{R}^{d}$ is a homotopy contracting $\mathbb{R}^{d}$ to $\{0\}$ if $\sigma_{x}(1)=x$ and $\sigma_{x}(0)=0$ for all $x \in \mathbb{R}^{d}$. We further say $\sigma$ is a follow the leader homotopy if $\sigma_{\sigma_{x}(t)}$ is a reparametrization of $\left.\sigma_{x}\right|_{[0, t]}$ for all $x \in \mathbb{R}^{d}$ and $t \in(0,1]$. [We will further assume that $t \rightarrow \sigma_{x}(t)$ is at least piecewise smooth.]

Example B.19. The radial homotopy, $\sigma$, is define by $\sigma_{x}(t)=$ tx for all $x \in \mathbb{R}^{d}$ and $t \in[0,1]$. This is a follow the leader homotopy.

In the main part of this paper we have secretly been using the following "complete axial homotopy" on $\mathbb{R}^{2}$, another follow the leader homotopy.

Notation B.20 (Complete axial homotopy). For any $x \in \mathbb{R}^{2}$, let $\sigma_{x}$ be the straight line path joining 0 to $\left(x_{1}, 0\right)$ followed by the straight line path joining $\left(x_{1}, 0\right) \rightarrow\left(x_{1}, x_{2}\right)=x$ as in Figure 12. We refer to this homotopy as the complete axial homotopy.

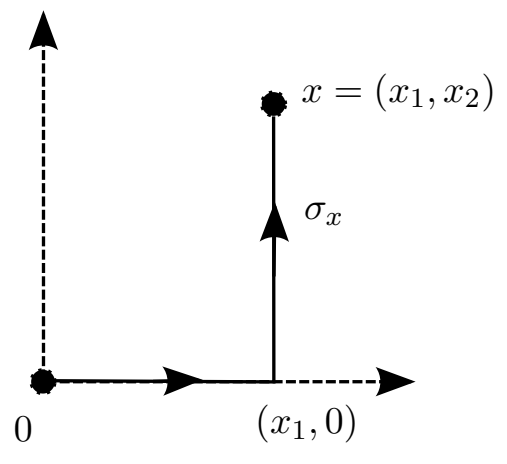

Figure 12. The taxi-cab path, $\varphi_{x}$, joining 0 to $x \in \mathbb{R}^{2}$.

Example B.21. If $\left\{\sigma_{x}: x \in \mathbb{R}^{d}\right\}$ is a homotopy contracting $\mathbb{R}^{d}$ to $\{0\}$, then $v_{\sigma}(A) \in \mathcal{G}$ defined by

$$
v_{\sigma}(A)(x):=\left[/ /{ }_{1}^{A}\left(\sigma_{x}\right)\right]^{-1} \text { for } x \in \mathbb{R}^{d}
$$

is a gauge on $\mathcal{A}$ because,

$$
\begin{aligned}
v_{\sigma}\left(A^{g}\right)(x) & =\left[/ /_{1}^{A^{g}}\left(\sigma_{x}\right)\right]^{-1}=\left[g^{-1}(x) / /_{1}^{A}\left(\sigma_{x}\right) g(0)\right]^{-1} \\
& =\left[/ /_{1}^{A}\left(\sigma_{x}\right)\right]^{-1} g(x)=v_{\sigma}(A)(x) g(x)=\left(v_{\sigma}(A) g\right)(x) .
\end{aligned}
$$

In this case we write $\mathcal{A}_{\sigma}$ for $\mathcal{A}_{v_{\sigma}}$ so that

$$
\mathcal{A}_{\sigma}:=v_{\sigma}^{-1}(\{I\})=\left\{A \in \mathcal{A}: /{ }_{1}^{A}\left(\sigma_{x}\right)=I \text { for all } x \in \mathbb{R}^{d}\right\} \subset \mathcal{A} .
$$


Definition B.22 (Homotopy gauges). If $\sigma$ is a homotopy contracting $\mathbb{R}^{d}$ to $\{0\}$ we refer to $v_{\sigma}$ in Eq. B.13) as homotopy gauge and $\mathcal{A}_{\sigma}$ in Eq. (B.14) as a homotopy slice.

Proposition B.23 (Follow the leader gauges). If $\sigma$ is a follow the leader homotopy and $A$ is a connection one form then the following are equivalent;

(1) $A$ is in the $\sigma$-gauge (i.e. $/{ }_{1}^{A}\left(\sigma_{x}\right)=I$ for all $\left.x \in \mathbb{R}^{d}\right)$,

(2) $/ / t_{t}^{A}\left(\sigma_{x}\right)=I$ for all $x \in \mathbb{R}^{d}$ and $t \in[0,1]$, and

(3) $A\left\langle\dot{\sigma}_{x}(t)\right\rangle=0$ for all $x \in \mathbb{R}^{d}$ and a.e. $t \in[0,1]$.

Consequently, by item 3. above,

$$
\mathcal{A}_{\sigma}=\left\{A \in \mathcal{A}: A\left\langle\dot{\sigma}_{x}(t)\right\rangle=0 \text { for all } x \in \mathbb{R}^{d} \text { and a.e. } t \in[0,1]\right\}
$$

is a linear slice for any follow the leader homotopy.

Proof. Since $/ /{ }^{A}(\sigma)$ invariant under reparametrizations of $\sigma$ it follows that for a follow the leader gauge,

$$
/ /{ }_{t}^{A}\left(\sigma_{x}\right)=/ /{ }_{1}^{A}\left(\sigma_{\sigma_{x}(t)}\right) \forall x \in \mathbb{R}^{d} \text { and } t \in[0,1]
$$

and this shows 1 . $\Longrightarrow 2$. To prove 2 . implies 3 . simply notice that

$$
0=\frac{d}{d t} I=\frac{d}{d t} / /_{t}^{A}\left(\sigma_{x}\right)=-A\left\langle\dot{\sigma}_{x}(t)\right\rangle / /_{t}^{A}\left(\sigma_{x}\right)=-A\left\langle\dot{\sigma}_{x}(t)\right\rangle
$$

The assertion $3 . \Longrightarrow 1$. is obvious since $/{ }_{t}^{A}\left(\sigma_{x}\right)$ satisfies

$$
0=\frac{d}{d t} / /_{t}^{A}\left(\sigma_{x}\right)+A\left\langle\dot{\sigma}_{x}(t)\right\rangle /{ }_{t}^{A}\left(\sigma_{x}\right)=\frac{d}{d t} / /_{t}^{A}\left(\sigma_{x}\right) .
$$

In what follows, if $x, v \in \mathbb{R}^{2}$, we let $v_{x} \in T \mathbb{R}^{d}$ be the tangent vector defined by

$$
v_{x} f:=\left(\partial_{v} f\right)(x)=\left.\frac{d}{d s}\right|_{0} f(x+s v)
$$

where $f$ is any differentiable function on $\mathbb{R}^{2}$.

Corollary B.24. If $\sigma$ is a follow the leader homotopy, $A \in \mathcal{A}_{\sigma}$, and $F=F^{A}$ is the curvature of $A$, then we can recover $A$ from $F$ using

$$
A\left\langle v_{x}\right\rangle=\int_{0}^{1} F\left\langle\dot{\sigma}_{x}(t), v_{x} \sigma_{(\cdot)}(\tau)\right\rangle d t \forall v_{x} \in T \mathbb{R}^{d}
$$

where explicitly,

$$
v_{x} \sigma_{(\cdot)}(\tau)=\partial_{v} \sigma_{x}(\tau)=\left.\frac{d}{d s}\right|_{0} \sigma_{(x+s v)}(\tau) .
$$

Proof. Using Cartan's formula while repeatedly using item 3. of Proposition B.23 shows

$$
d A\left\langle\dot{\sigma}_{x}(t), \partial_{v} \sigma_{x}(t)\right\rangle=\frac{d}{d t} A\left\langle\partial_{v} \sigma_{x}(t)\right\rangle-\partial_{v} A\left\langle\dot{\sigma}_{x}(t)\right\rangle=\frac{d}{d t} A\left\langle\partial_{v} \sigma_{x}(t)\right\rangle
$$

and

$$
(A \wedge A)\left\langle\dot{\sigma}_{x}(t), \partial_{v} \sigma_{x}(t)\right\rangle=\left[A\left\langle\dot{\sigma}_{x}(t)\right\rangle, A\left\langle\partial_{v} \sigma_{x}(t)\right\rangle\right]=0 .
$$

Therefore we may conclude that

$$
F\left\langle\dot{\sigma}_{x}(t), \partial_{v} \sigma_{x}(t)\right\rangle=\frac{d}{d t} A\left\langle\partial_{v} \sigma_{x}(t)\right\rangle .
$$


Integrating this expression on $t$ while using $\partial_{v} \sigma_{x}(0)=\partial_{v} 0=0$ and $\partial_{v} \sigma_{x}(1)=\partial_{v} x=v_{x}$ gives Eq. B.15.

Let us generalize the previous result in order to compute $\pi_{\sigma}(A)$ for an arbitrary $A \in \mathcal{A}$.

Theorem B.25. If $\sigma$ is a follow the leader homotopy and $A \in \mathcal{A}$, then

$$
\pi_{\sigma}(A)\left\langle v_{x}\right\rangle=\operatorname{Ad}_{g_{A}(x)^{-1}} \int_{0}^{1} \operatorname{Ad}_{/ /{ }_{1 \leftarrow \tau}^{A}\left(\sigma_{x}\right)} F^{A}\left(\dot{\sigma}_{x}(\tau), v_{x} \sigma_{(\cdot)}(\tau)\right) d \tau
$$

where $\pi_{\sigma}(A):=A^{g_{A}}$ with $g_{A}(x):=/ /_{1}^{A}\left(\sigma_{x}(\cdot)\right)$ and

$$
/ /{ }_{1 \leftarrow \tau}^{A}\left(\sigma_{x}\right):=/ /_{1}^{A}\left(\sigma_{x}\right) / /_{\tau}^{A}\left(\sigma_{x}\right)^{-1} .
$$

Proof. Let $v_{x} \in T_{x} \mathbb{R}^{d}$ and $x(s) \in \mathbb{R}^{d}$ such that $x^{\prime}(0)=v_{x}$ and in particular $x(0)=x$. We are now going to apply Proposition A.7 with $\ell_{s}(t)=\sigma_{x(s)}(t)$. First observe that

$$
d g_{A}\left(v_{x}\right)+A\left\langle v_{x}\right\rangle g_{A}(x)=\left.\frac{\nabla}{d s}\right|_{0} g_{A}(x(s))=\left.\frac{\nabla}{d s}\right|_{0} / /_{1}^{A}\left(\sigma_{x(s)}\right)=\left.\frac{\nabla}{d s}\right|_{0} / /_{1}^{A}\left(\ell_{s}\right)
$$

and, by Eq. A.10 of Proposition A.7.

$$
\begin{aligned}
\left.\frac{\nabla}{d s}\right|_{0} / /_{1}^{A}\left(\ell_{s}\right) & =/ /_{1}^{A}\left(\ell_{0}\right) \int_{0}^{1} \operatorname{Ad}_{/ /{ }_{\tau}^{A}\left(\ell_{0}\right)^{-1}} F^{A}\left(\dot{\ell}_{0}(\tau), \ell_{0}^{\prime}(\tau)\right) d \tau \\
& =g_{A}(x) \int_{0}^{1} \operatorname{Ad}_{/ /{ }_{\tau}^{A}\left(\sigma_{x}\right)^{-1}} F^{A}\left(\dot{\sigma}_{x}(\tau), v_{x} \sigma_{(\cdot)}(\tau)\right) d \tau \\
& =g_{A}(x) \int_{0}^{1} \operatorname{Ad}_{g_{A}\left(\sigma_{x}(\tau)\right)^{-1}} F^{A}\left(\dot{\sigma}_{x}(\tau), v_{x} \sigma_{(\cdot)}(\tau)\right) d \tau .
\end{aligned}
$$

Combining these two identities and multiplying the result on the left by $g_{A}(x)^{-1}$ gives,

$$
\begin{aligned}
B\left\langle v_{x}\right\rangle & =A^{g_{A}}\left\langle v_{x}\right\rangle=\int_{0}^{1} \operatorname{Ad}_{g_{A}\left(\sigma_{x}(\tau)\right)^{-1}} F^{A}\left(\dot{\sigma}_{x}(\tau), v_{x} \sigma_{(\cdot)}(\tau)\right) d \tau \\
& =\operatorname{Ad}_{g_{A}(x)^{-1}} \int_{0}^{1} \operatorname{Ad}_{g_{A}(x)} \operatorname{Ad}_{g_{A}\left(\sigma_{x}(\tau)\right)^{-1} F^{A}}\left(\dot{\sigma}_{x}(\tau), v_{x} \sigma_{(\cdot)}(\tau)\right) d \tau .
\end{aligned}
$$

Finally we have $g_{A}(x) g_{A}\left(\sigma_{x}(\tau)\right)^{-1}=/ /_{1 \leftarrow \tau}^{A}\left(\sigma_{x}\right)$ so that

$$
A^{g_{A}}\left\langle v_{x}\right\rangle=\operatorname{Ad}_{g_{A}(x)^{-1}} \int_{0}^{1} \operatorname{Ad}_{/ / /_{1 \leftarrow \tau}^{A}\left(\sigma_{x}\right)} F^{A}\left(\dot{\sigma}_{x}(\tau), v_{x} \sigma_{(\cdot)}(\tau)\right) d \tau
$$

Remark B.26. If we take $v_{x}=\frac{d}{d s} \sigma_{y}(s)$ for some $y \in \mathbb{R}^{d}$ and $s \in[0,1]$ (so that $x=\sigma_{y}(s)$ ), then

$$
\dot{\sigma}_{x}(\tau)=\frac{d}{d \tau} \sigma_{\sigma_{y}(s)}(\tau) \text { and } v_{x} \sigma_{(\cdot)}(\tau)=\frac{d}{d s} \sigma_{\sigma_{y}(s)}(\tau)
$$

are parallel by the follow the leader property so that $F^{A}\left(\dot{\sigma}_{x}(\tau), v_{x} \sigma_{(\cdot)}(\tau)\right)=0$ for a.e. $\tau$ in this case. This shows explicitly that right side of Eq. B.16) is indeed in $\mathcal{A}_{\sigma}$. 
Corollary B.27. If $A \in \mathcal{A}_{\sigma}$ and $\eta \in \mathcal{A}$, then

$$
\begin{aligned}
\pi_{\sigma}(A+\eta)\left\langle v_{x}\right\rangle & =(A+\eta)^{g_{\eta}}\left\langle v_{x}\right\rangle=\left[\operatorname{Ad}_{g_{\eta}^{-1}} A+\pi_{\sigma}(\eta)\right]\left\langle v_{x}\right\rangle \\
& =\operatorname{Ad}_{g_{\eta}^{-1}(x)}\left[A\left\langle v_{x}\right\rangle+\int_{0}^{1} \operatorname{Ad}_{/ / /_{1 \leftarrow \tau}^{\eta}\left(\sigma_{x}\right)} F^{\eta}\left(\dot{\sigma}_{x}(\tau), v_{x} \sigma_{(\cdot)}(\tau)\right) d \tau\right] .
\end{aligned}
$$

Proof. As $A \in \mathcal{A}_{\sigma}$ we know that $A\left\langle\dot{\sigma}_{x}(t)\right\rangle=0$ for a.e. $t$ and therefore,

$$
g_{A+\eta}(x)=/ /_{1}^{A+\eta}\left(\sigma_{x}\right)=/ /_{1}^{\eta}\left(\sigma_{x}\right)=g_{\eta}(x)
$$

and hence

$$
\begin{aligned}
\pi_{\sigma}(A+\eta) & =(A+\eta)^{g_{\eta}}=\operatorname{Ad}_{g_{\eta}^{-1}}(A+\eta)+g_{\eta}^{-1} d g_{\eta} \\
& =\operatorname{Ad}_{g_{\eta}^{-1}} A+\operatorname{Ad}_{g_{\eta}^{-1} \eta}+g_{\eta}^{-1} d g_{\eta}=\operatorname{Ad}_{g_{\eta}^{-1}} A+\pi_{\sigma}(\eta)
\end{aligned}
$$

which gives Eq. (B.17). Making use of Theorem B.25 with $A$ replaced by $\eta$ to evaluate $\pi_{\sigma}(\eta)$ in Eq. B.17) then gives Eq. B.18.

Meta-Proposition B.28. If $\sigma$ is a follow the leader homotopy, $\eta \in \mathcal{A}$, and $\Psi: \mathcal{A} \rightarrow[0, \infty]$ is a function such that $\Psi\left(A^{g_{\eta}}\right)=\Psi(A)$ for all $A \in \mathcal{A}$, then

$$
\int_{\mathcal{A}_{\sigma}} \Psi(A+\eta) d m_{\sigma}(A)=\int_{\mathcal{A}_{\sigma}} \Psi(A) d m_{\sigma}(A) .
$$

[Note that $\eta$ is not assumed to be in $\mathcal{A}_{\sigma}$ and so we can not directly prove Eq. (B.19]) by invoking translation invariance of $m_{\sigma}$.]

Proof. If $A \in \mathcal{A}_{\sigma}$ and $\eta \in \mathcal{A}$, then $A+\eta \in \mathcal{A}$ and so by assumption and

$$
\Psi(A+\eta)=\Psi\left([A+\eta]^{g_{\eta}}\right)=\Psi\left(\operatorname{Ad}_{g_{\eta}^{-1}} A+\pi_{\sigma}(\eta)\right) .
$$

As we have already explained, $A \rightarrow \operatorname{Ad}_{g_{\eta}^{-1}} A+\pi_{\sigma}(\eta)$ is a rotation followed by a translation which preserves Lebesgue measure and $m_{\sigma}$ is a Lebesgue measure. Thus, it follows that

$$
\int_{\mathcal{A}_{\sigma}} \Psi(A+\eta) d m_{\sigma}(A)=\int_{\mathcal{A}_{\sigma}} \Psi\left(\operatorname{Ad}_{g_{\eta}^{-1}} A+\pi_{\sigma}(\eta)\right) d m_{\sigma}(A)=\int_{\mathcal{A}_{\sigma}} \Psi(A) d m_{\sigma}(A) .
$$

Meta-Corollary B.29. If $\sigma$ is a follow the leader homotopy, $\eta \in \mathcal{A}$, and $\Psi: \mathcal{A} \rightarrow[0, \infty]$ is a function such that $\Psi\left(A^{g_{s \eta}}\right)=\Psi(A)$ for all $A \in \mathcal{A}$ and $s \in(-\varepsilon, \varepsilon)$ for some $\varepsilon>0$, then

$$
\int_{\mathcal{A}_{\sigma}}\left(\partial_{\eta} \Psi\right)(A) d m_{\sigma}(A)=0
$$

Proof. By Proposition B.28,

$$
\int_{\mathcal{A}_{\sigma}} \Psi(A) d m_{\sigma}(A)=\int_{\mathcal{A}_{\sigma}} \Psi(A+s \eta) d m_{\sigma}(A) \forall s \in(-\varepsilon, \varepsilon) .
$$

Differentiating this equation at $s=0$ then gives Eq. B.20). 
Remark B.30. Warning: even if $\Psi$ is gauge invariant, it is quite unlikely that $\partial_{\eta} \Psi$ will still be gauge invariant since in general we have,

$$
\begin{aligned}
\left(\partial_{\eta} \Psi\right)\left(A^{g}\right) & =\left.\frac{d}{d t}\right|_{0} \Psi\left(A^{g}+t \eta\right)=\left.\frac{d}{d t}\right|_{0} \Psi\left(\left(A^{g}+t \eta\right)^{g^{-1}}\right) \\
& =\left.\frac{d}{d t}\right|_{0} \Psi\left(A+t \operatorname{Ad}_{g} \eta\right)=\left(\partial_{\operatorname{Ad}_{g} \eta} \Psi\right)(A) .
\end{aligned}
$$

So in order for $\partial_{\eta} \Psi$ to be gauge invariant we would typically need $\operatorname{Ad}_{g} \eta=\eta$ for all $g \in \mathcal{G}$ which would force $\eta$ to take values in the center of $\mathfrak{k}$. On the other hand, for any $g \in \mathcal{G}$ such that $\operatorname{Ad}_{g} \eta=\eta$ we will have

$$
\left(\partial_{\eta} \Psi\right)\left(A^{g}\right)=\left(\partial_{\eta} \Psi\right)(A) \text { for all } A \in \mathcal{A} .
$$

Notation B.31 ( $\sigma$-fixed $Y M$ "measures"). To each follow the leader homotopy, $\sigma$, let $\mu_{\sigma}$ be the formal probability measure on $\mathcal{A}_{\sigma}$ given by,

$$
d \mu_{\sigma}(A)=\frac{1}{Z_{\sigma}} e^{-\frac{1}{2}\left\|F^{A}\right\|^{2}} d m_{\sigma}(A) .
$$

Meta-Corollary B.32. If $\sigma$ is a follow the leader homotopy, $\eta \in \mathcal{A}$, and $\Psi: \mathcal{A} \rightarrow[0, \infty]$ is a function such that $\Psi\left(A^{g_{s \eta}}\right)=\Psi(A)$ for all $A \in \mathcal{A}$ and $s \in(-\varepsilon, \varepsilon)$ for some $\varepsilon>0$, then

$$
\int_{\mathcal{A}_{\sigma}}\left(\partial_{\eta} \Psi\right)(A) d \mu_{\sigma}(A)=\int_{\mathcal{A}_{\sigma}} \Psi(A) \cdot\left\langle d^{A} \eta, F^{A}\right\rangle d \mu_{\sigma}(A)
$$

where

$$
\left(d^{A} \eta\right)_{i j}=\nabla_{i}^{A} \eta_{j}-\nabla_{j}^{A} \eta_{i} \text { and } \nabla_{i}^{A} \eta_{j}:=\partial_{i} \eta_{j}+\operatorname{ad}_{A_{i}} \eta_{j} .
$$

Warning: gauge invariance has been broken in Eq. (B.21) which holds for all follow the leader homotopies, $\sigma$, but both sides of this equation may very well depend on the choice of $\sigma$.

Proof. Since $A \rightarrow e^{-\frac{1}{2}\left\|F^{A}\right\|^{2}}$ is gauge invariant we may apply Meta-Corollary B.29 with $\Psi$ replaced by $A \rightarrow \Psi(A) e^{-\frac{1}{2}\left\|F^{A}\right\|^{2}}$ in order to find,

$$
\begin{aligned}
0 & =\frac{1}{Z_{\sigma}} \int_{\mathcal{A}_{\sigma}} \partial_{\eta}\left[A \rightarrow \Psi(A) e^{-\frac{1}{2}\left\|F^{A}\right\|^{2}}\right] d m_{\sigma}(A) \\
& =\frac{1}{Z_{\sigma}} \int_{\mathcal{A}_{\sigma}}\left[\left(\partial_{\eta} \Psi\right)(A)-\frac{1}{2} \partial_{\eta}\left\|F^{A}\right\|^{2}\right] e^{-\frac{1}{2}\left\|F^{A}\right\|^{2}} d m_{\sigma}(A)
\end{aligned}
$$

wherein we have used the product and the chain rule for the second equality. This completes the proof since,

$$
\frac{1}{2} \partial_{\eta}\left\|F^{A}\right\|^{2}=\left\langle\partial_{\eta} F^{A}, F^{A}\right\rangle
$$

and

$$
\partial_{\eta} F_{i j}^{A}=\partial_{\eta}\left(\partial_{i} A_{j}-\partial_{j} A_{i}+\left[A_{i}, A_{j}\right]\right)=\partial_{i} \eta_{j}-\partial_{j} \eta_{i}+\left[\eta_{i}, A_{j}\right]+\left[A_{i}, \eta_{j}\right]=\nabla_{i}^{A} \eta_{j}-\nabla_{j}^{A} \eta_{i} .
$$

Notation B.33. To each follow the leader homotopy, $\sigma$, and $\eta \in \mathcal{A}$ let $u_{\eta}^{\sigma}: \mathbb{R}^{d} \rightarrow \mathfrak{k}$ be defined by

$$
u_{\eta}^{\sigma}(x):=\int_{0}^{1} \eta\left\langle\dot{\sigma}_{x}(\tau)\right\rangle d \tau .
$$


Remark B.34. Since $\sigma$ is a follow the leader homotopy we have,

$$
u_{\eta}^{\sigma}\left(\sigma_{x}(t)\right)=\int_{0}^{1} \eta\left\langle\dot{\sigma}_{\sigma_{x}(t)}(\tau)\right\rangle d \tau=\int_{\left.\sigma_{x}\right|_{[0, t]}} \eta=\int_{0}^{t} \eta\left\langle\dot{\sigma}_{x}(\tau)\right\rangle d \tau
$$

and therefore,

$$
d u_{\eta}^{\sigma}\left(\dot{\sigma}_{x}(t)\right)=\frac{d}{d t} u_{\eta}^{\sigma}\left(\sigma_{x}(t)\right)=\frac{d}{d t} \int_{0}^{t} \eta\left\langle\dot{\sigma}_{x}(\tau)\right\rangle d \tau=\eta\left\langle\dot{\sigma}_{x}(t)\right\rangle .
$$

Proposition B.35 (Projected vector fields). If $A \in \mathcal{A}_{\sigma}$ and $A, \eta \in \mathcal{A}$, then

$$
d \pi_{\sigma}\left\langle\eta_{A}\right\rangle:=\left.\frac{d}{d s}\right|_{0} \pi_{\sigma}(A+s \eta)=-\operatorname{ad}_{u_{\eta}^{\sigma}} A+\eta-d u_{\eta}^{\sigma} .
$$

[As is seen directly from $E q$. B.22), $\eta-d u_{\eta}^{\sigma} \in \mathcal{A}_{\sigma}$ for all $\eta \in \mathcal{A}$.]

Proof. Let $v_{x} \in T_{x} \mathbb{R}^{d}$. Replace $\eta$ by $s \eta$ in Eq. B.18) and then differentiate the result with respect to $s$ to find,

$$
\begin{aligned}
\left(d \pi_{\sigma}\left\langle\eta_{A}\right\rangle\right)\left(\left\langle v_{x}\right\rangle\right) & =\left.\frac{d}{d s}\right|_{0} \pi_{\sigma}(A+s \eta)\left\langle v_{x}\right\rangle \\
& =\left.\frac{d}{d s}\right|_{0}\left(\operatorname{Ad}_{g_{s \eta}^{-1}(x)}\left[A\left\langle v_{x}\right\rangle+\int_{0}^{1} \operatorname{Ad}_{/ /{ }_{1 \leftarrow \tau}^{s \eta}\left(\sigma_{x}\right)} F^{s \eta}\left(\dot{\sigma}_{x}(\tau), v_{x} \sigma_{(\cdot)}(\tau)\right) d \tau\right]\right) \\
& =\left(\left.\frac{d}{d s}\right|_{0} \operatorname{Ad}_{g_{s \eta}^{-1}(x)}\right) A\left\langle v_{x}\right\rangle+\left.\int_{0}^{1} \frac{d}{d s}\right|_{0} F^{s \eta}\left(\dot{\sigma}_{x}(\tau), v_{x} \sigma_{(\cdot)}(\tau)\right) d \tau \\
& =\left(\left.\frac{d}{d s}\right|_{0} \operatorname{Ad}_{g_{s \eta}^{-1}(x)}\right) A\left\langle v_{x}\right\rangle+\int_{0}^{1} d \eta\left(\dot{\sigma}_{x}(\tau), v_{x} \sigma_{(\cdot)}(\tau)\right) d \tau .
\end{aligned}
$$

Choosing $x(s) \in \mathbb{R}^{d}$ so that $x^{\prime}(0)=v_{x}$ and using

$$
\begin{aligned}
\left.\int_{0}^{1} d \eta\left(\dot{\sigma}_{x}(\tau), \sigma_{x(s)}^{\prime}(\tau)\right) d \tau\right|_{s=0} & =\left.\int_{0}^{1}\left[\frac{d}{d \tau} \eta\left(\sigma_{x(s)}^{\prime}(\tau)\right)-\frac{d}{d s} \eta\left(\dot{\sigma}_{x(s)}(\tau)\right)\right] d \tau\right|_{s=0} \\
& =\eta\left\langle v_{x}\right\rangle-\left.\int_{0}^{1} \frac{d}{d s}\right|_{0} \eta\left(\dot{\sigma}_{x(s)}(\tau)\right) d \tau \\
& =\eta\left\langle v_{x}\right\rangle-\left.\frac{d}{d s}\right|_{0} u_{\eta}^{\sigma}(x(s))=\left(\eta-d u_{\eta}^{\sigma}\right)\left\langle v_{x}\right\rangle
\end{aligned}
$$

and so

$$
d \pi_{\sigma}\left\langle\eta_{A}\right\rangle=\left(\left.\frac{d}{d s}\right|_{0} \operatorname{Ad}_{g_{s \eta}^{-1}(x)}\right) A+\eta-d u_{\eta}^{\sigma}
$$

Moreover, since

$$
\frac{d}{d t} / /_{t}^{s \eta}\left(\sigma_{x}\right)=-s \eta\left\langle\dot{\sigma}_{x}(t)\right\rangle / /_{t}^{s \eta}\left(\sigma_{x}\right) \text { with } / /_{0}^{s \eta}\left(\sigma_{x}\right)=I
$$

we conclude that

$$
\left.\frac{d}{d t} \frac{d}{d s}\right|_{0} /\left.\right|_{t} ^{s \eta}\left(\sigma_{x}\right)=\left.\frac{d}{d s}\right|_{0} \frac{d}{d t} /\left.\right|_{t} ^{s \eta}\left(\sigma_{x}\right)=\left.\frac{d}{d s}\right|_{0}\left[-s \eta\left\langle\dot{\sigma}_{x}(t)\right\rangle /\left.\right|_{t} ^{s \eta}\left(\sigma_{x}\right)\right]=\eta\left\langle\dot{\sigma}_{x}(t)\right\rangle .
$$


Integrating this equation in $t$ shows

$$
\left.\frac{d}{d s}\right|_{0} g_{s \eta}(x)=\left.\frac{d}{d s}\right|_{0} / /_{1}^{s \eta}\left(\sigma_{x}\right)=\int_{0}^{1} \eta\left\langle\dot{\sigma}_{x}(\tau)\right\rangle d \tau=u_{\eta}^{\sigma}(x)
$$

and hence $\left.\frac{d}{d s}\right|_{0} \operatorname{Ad}_{g_{s \eta}^{-1}(x)}=-\operatorname{ad}_{u_{\eta}^{\sigma}(x)}$ which combined with Eq. B.25 gives Eq. B.23.

Example B.36. Let us work out $u_{\eta}^{\sigma}$ in the special case where $d=2, \sigma$ is the complete axial homotopy, and $\eta=\eta_{1} d x$. In this case,

$$
u_{\eta}^{\sigma}(x, y)=u_{\eta}^{\sigma}(x, 0)=\int_{0}^{1} \eta\left\langle\dot{\sigma}_{(x, 0)}(\tau)\right\rangle d \tau=\int_{0}^{x} \eta_{1}(s, 0) d s
$$

and therefore

$$
\eta-d u_{\eta}^{\sigma}=\left[\eta_{1}(x, y)-\eta_{1}(x, 0)\right] d x=\bar{\eta}_{1}(x, 0) d x
$$

which agrees with formulas we have used in the body of this paper.

Corollary B.37. If $\sigma$ is a follow the leader homotopy, $\Psi$ is a smooth gauge invariant function on $\mathcal{A}$, and $\eta \in \mathcal{A}$, then

$$
\left(\partial_{\eta} \Psi\right)(A)=\left(\partial_{\left[-\operatorname{ad}_{u_{\eta}^{\sigma}} A+\left(\eta-d u_{\eta}^{\sigma}\right)\right]} \Psi\right)(A) \forall A \in \mathcal{A}_{\sigma} .
$$

Proof. By gauge invariance of $\Psi, \Psi(A+s \eta)=\Psi\left(\pi_{\sigma}(A+s \eta)\right)$ and therefore using Proposition B.35.

$$
\begin{aligned}
\left(\partial_{\eta} \Psi\right)(A) & =\left.\frac{d}{d s}\right|_{0} \Psi(A+s \eta)=\left.\frac{d}{d s}\right|_{0} \Psi\left(\pi_{\sigma}(A+s \eta)\right) \\
& =\left(\partial_{\left[-\operatorname{ad}_{u_{\eta}^{\sigma}} A+\left(\eta-d u_{\eta}^{\sigma}\right)\right]} \Psi\right)(A) .
\end{aligned}
$$

Lemma B.38. If $d=2, \sigma$ is a follow the leader homotopy, and $A \in \mathcal{A}_{\sigma}$, then $F^{A}=d A$.

Proof. The point is that $A \wedge A$ is determined by its value on any two linearly independent vectors, $\left\{u_{1}, u_{2}\right\}$. We may always take $u_{1}=\dot{\sigma}_{x}(1)$ in which case

$$
A \wedge A\left\langle u_{1}, u_{2}\right\rangle=\left[A\left\langle u_{1}\right\rangle, A\left\langle u_{2}\right\rangle\right]=\left[A\left\langle\dot{\sigma}_{x}(1)\right\rangle, A\left\langle u_{2}\right\rangle\right]=0 .
$$

Remark B.39. If $\sigma$ is a follow the leader homotopy and $g \in \mathcal{G}$, then $\operatorname{Ad}_{g^{-1}}$ preserves $\mathcal{A}_{\sigma}$. Indeed if $A \in \mathcal{A}_{\sigma}$, then $\operatorname{Ad}_{g} A \in \mathcal{A}_{\sigma}$ since

$$
\left(\operatorname{Ad}_{g} A\right)\left\langle\dot{\sigma}_{x}(t)\right\rangle:=\operatorname{Ad}_{g\left(\sigma_{x}(t)\right)}\left[A\left\langle\dot{\sigma}_{x}(t)\right\rangle\right]=0 \forall x \in \mathbb{R}^{d} \text { \& a.e. } t \in[0,1] .
$$

Meta-Proposition B.40. Let $m$ denote formal Lebesgue measure on $\mathcal{A}$ and $\sigma$ be a follow the leader homotopy. Then the formal measure, $m_{\sigma}=m_{v_{\sigma}}$, given by Proposition B.9 is a Lebesgue measure on $\mathcal{A}_{\sigma}$.

Meta-Proof. Since, for $g \in \mathcal{G}, \operatorname{Ad}_{g^{-1}}$ acts orthogonally on $\mathcal{A}$ equipped with the $L^{2}$-norm and hence we (heuristically) have $\operatorname{Det}\left(\operatorname{Ad}_{g^{-1}}\right)=1$ and so $A \rightarrow A^{g}=\operatorname{Ad}_{g^{-1}} A+g^{-1} d g$ is (formally) an affine action. Combining this observation with Remark B.39 allows us to formally apply Theorem B.10 in this infinite dimensional context. 
Meta-Corollary B.41. Let $m$ denote formal Lebesgue measure on $\mathcal{A}$ and $\sigma$ be a follow the leader homotopy then (recall Definition B.11)

$$
f_{\mathcal{A}} \Psi(A) d m(A)=\int_{\mathcal{A}_{\sigma}} \Psi(A) d m_{\sigma}(A)
$$

where $m_{\sigma}$ is a Lebesgue measure on $\mathcal{A}_{\sigma}$.

To apply this last result to the formal $Y M$-measures we need the following simple lemma.

Lemma B.42. The function, $\mathcal{A} \ni A \rightarrow\left\|F^{A}\right\|$ as described in Eq. (1.4) is invariant under the full gauge group.

Proof. From Theorem A.1, we know that $F^{A^{g}}=\operatorname{Ad}_{g^{-1}} F^{A}$ and since $|\cdot|_{\mathfrak{k}}$ is assumed to be $\operatorname{Ad}_{K^{-}}$ invariant we find, for any $g \in C^{1}\left(\mathbb{R}^{d}, K\right)$, then

$$
\sum_{i<j}\left|F^{A^{g}}\left\langle e_{i}, e_{j}\right\rangle(x)\right|_{\mathfrak{k}}^{2}=\sum_{i<j}\left|\operatorname{Ad}_{g(x)^{-1}} F^{A}\left\langle e_{i}, e_{j}\right\rangle(x)\right|_{\mathfrak{k}}^{2}=\sum_{i<j}\left|F^{A}\left\langle e_{i}, e_{j}\right\rangle(x)\right|_{\mathfrak{k}}^{2} .
$$

Integrating this equation over $\mathbb{R}^{d}$ immediately gives $\left\|F^{A^{g}}\right\|^{2}=\left\|F^{A}\right\|^{2}$.

Definition B.43 (Formal Yang-Mills Expectations). If $\Psi: \mathcal{A} \rightarrow \mathbb{C}$ is a restricted gauge invariant function, we define

$$
\langle\Psi\rangle_{Y M}:=\frac{1}{Z_{\sigma}} \int_{\mathcal{A}_{\sigma}} \Psi(A) e^{-\frac{1}{2}\left\|F^{A}\right\|^{2}} d \tilde{m}_{\sigma}(A),
$$

where $\sigma$ is any follow the leader homotopy, $\tilde{m}_{\sigma}$ is a formal Lebesgue measure on $\mathcal{A}_{\sigma}$, and (formally)

$$
Z_{\sigma}:=\int_{\mathcal{A}_{\sigma}} e^{-\frac{1}{2}\left\|F^{A}\right\|^{2}} d \tilde{m}_{\sigma}(A)
$$

A few remarks are in order;

(1) The expression in Eq. B.26) is formally independent of the choice of Lebesgue measure on $\mathcal{A}_{\sigma}$ since they all differ by a multiplicative constant and any such multiplicative constant will also occur in the normalization constant, $Z_{\sigma}$.

(2) The expression in Eq. (B.26) is formally independent of the choice of the follow the leader homotopy, $\sigma$, used in the definition since by the first remark we may choose $\tilde{m}_{\sigma}=m_{\sigma}$ in which case

$$
\langle\Psi\rangle_{Y M}:=\frac{1}{Z} f_{\mathcal{A}} \Psi(A) e^{-\frac{1}{2}\left\|F^{A}\right\|^{2}} d m(A)
$$

with

$$
Z=f_{\mathcal{A}} e^{-\frac{1}{2}\left\|F^{A}\right\|^{2}} d m(A)
$$

Our final goal is to show (formally) that $\langle\Psi\rangle_{Y M_{2}}$ is invariant under area preserving diffeomorphisms. 
B.5. Area preserving diffeomorphisms. Let $\sigma$ be a homotopy contracting $\mathbb{R}^{d}$ to $\{0\}$.

Notation B.44 (Diffeomorphism action on $\mathcal{A}_{\sigma}$ ). If $\varphi: \mathbb{R}^{d} \rightarrow \mathbb{R}^{d}$ is a diffeomorphism, let $\hat{\varphi}_{\sigma}$ : $\mathcal{A}_{\sigma} \rightarrow \mathcal{A}_{\sigma}$ be defined by

$$
\hat{\varphi}_{\sigma}(A):=\pi_{\sigma}\left(\varphi^{*} A\right)=\left(\varphi^{*} A\right)^{g_{A}} \text { for all } A \in \mathcal{A}_{\sigma},
$$

where

$$
g_{A}(p)=/ /_{1}^{A}\left(\varphi \circ \sigma_{p}\right) \text { for all } p \in \mathbb{R}^{d} .
$$

Proposition B.45 (The diffeomorphism action parallel translation). If $\varphi: \mathbb{R}^{d} \rightarrow \mathbb{R}^{d}$ is a diffeomorphism, $A \in \mathcal{A}_{\sigma}$, and $\alpha \in C^{1}\left([a, b], \mathbb{R}^{2}\right)$ is a path, then

$$
/ / \hat{\varphi}_{\sigma}(A)(\alpha)=g_{A}(\alpha(b))^{-1} / /{ }^{A}(\varphi \circ \alpha) g_{A}(\alpha(a))
$$

where $g_{A}$ is as in Eq. (B.28).

Proof. Using Theorem A.1 and Proposition A.3 we have

$$
\begin{aligned}
& / /^{\hat{\varphi}_{\sigma}(A)}(\alpha)=/ /\left[\varphi^{*} A\right]^{g_{A}}(\alpha)=g_{A}(\alpha(b))^{-1} / /\left[\varphi^{*} A\right](\alpha) g_{A}(\alpha(a)) \\
& =g_{A}(\alpha(b))^{-1} / /^{A}(\varphi \circ \alpha) g_{A}(\alpha(a)) \text {. }
\end{aligned}
$$

For the rest of this appendix we now exclusively assume that $d=2$ and further assume that $\varphi: \mathbb{R}^{2} \rightarrow \mathbb{R}^{2}$ is an area preserving diffeomorphism.

Definition B.46. A diffeomorphism, $\varphi: \mathbb{R}^{2} \rightarrow \mathbb{R}^{2}$ is area preserving provided $\left|\operatorname{det} \varphi^{\prime}(p)\right|=1$ for all $p \in \mathbb{R}^{2}$. We further let $\varepsilon(\varphi)=\operatorname{sgn}\left(\operatorname{det} \varphi^{\prime}\right) \in\{ \pm 1\}$ so that $\varphi$ is orientation preserving if $\varepsilon(\varphi)=1$ and orientation reversing if $\varepsilon(\varphi)=-1$. Alternatively stated, a diffeomorphism. $\varphi: \mathbb{R}^{2} \rightarrow \mathbb{R}^{2}$, is area preserving iff $\varphi^{*}(d x \wedge d y)=\varepsilon(\varphi) d x \wedge d y$ where $\varepsilon(\varphi)$ is either 1 or -1 . i.e.

Our final goal of this appendix is to "prove" the following Meta-Theorem.

Meta-Theorem B.47. Let $\varphi: \mathbb{R}^{2} \rightarrow \mathbb{R}^{2}$ be an area preserving diffeomorphism and $\Psi: \mathcal{A} \rightarrow$ $[0, \infty]$ be a function. If either;

(1) $\varphi(0)=0$ and $\Psi$ is a restricted gauge invariant, or

(2) $\Psi$ is invariant under the full gauge group,

then

$$
\left\langle\Psi \circ \varphi^{*}\right\rangle_{Y M_{2}}=\langle\Psi\rangle_{Y M_{2}}
$$

Meta-Proof. This result follows from using either Meta-Theorem B.55 or Meta-Theorem B.56 below along with the observation in the next lemma that $\mathcal{A} \ni A \rightarrow\left\|F^{A}\right\|$ is invariant under $\varphi^{*}$.

Lemma B.48. If $\varphi: \mathbb{R}^{2} \rightarrow \mathbb{R}^{2}$ is an area preserving diffeomorphism, then $\left\|F^{\varphi^{*} A}\right\|=\left\|F^{A}\right\|$ for all $A \in \mathcal{A}$.

Proof. Writing $F^{A}=f^{A} d x_{1} \wedge d x^{2}$ we have

$$
\begin{aligned}
f^{\varphi^{*} A} d x_{1} \wedge d x^{2} & =F^{\varphi^{*} A}=\varphi^{*} F^{A}=f^{A} \circ \varphi \cdot \varphi^{*}\left(d x_{1} \wedge d x_{2}\right) \\
& =\varepsilon(\varphi) f^{A} \circ \varphi \cdot d x_{1} \wedge d x_{2}
\end{aligned}
$$


where $\varepsilon(\varphi) \in\{ \pm 1\}$ since $\varphi$ is area preserving and consequently,

$$
\begin{aligned}
\left\|F^{\varphi^{*} A}\right\|^{2} & =\int_{\mathbb{R}^{2}}\left|f^{\varphi^{*} A}(x)\right|_{\mathfrak{k}}^{2} d x=\int_{\mathbb{R}^{2}}\left|f^{A}(\varphi(x))\right|_{\mathfrak{k}}^{2} d x \\
& =\int_{\mathbb{R}^{2}}\left|f^{A}(x)\right|_{\mathfrak{k}}^{2} d x=\left\|F^{A}\right\|^{2} .
\end{aligned}
$$

So it now remains to "prove" Meta-Theorem B.55 and Meta-Theorem B.56 below. In brief these theorems assert; if $\varphi: \mathbb{R}^{2} \rightarrow \mathbb{R}^{2}$ is an area preserving diffeomorphism and $\Psi: \mathcal{A} \rightarrow[0, \infty]$ is a function, then

$$
f_{\mathcal{A}} \Psi\left(\varphi^{*} A\right) d m(A)=f_{\mathcal{A}} \Psi(A) d m(A),
$$

provided $\Psi$ is invariant under the full gauge group (Meta-Theorem B.56) or $\varphi(0)=0$ and $\Psi$ is invariant under the restricted gauge group (Meta-Theorem B.55). Let us note that when $\varphi(0) \neq 0$, $\varphi^{*} g=g \circ \varphi$ will not be in $\mathcal{G}$ for $g \in \mathcal{G}$. Nevertheless, if $\Psi$ is invariant under the full gauge group, then

$$
\Psi\left(\varphi^{*} A^{g}\right)=\Psi\left(\left(\varphi^{*} A\right)^{\varphi^{*} g}\right)=\Psi\left(\varphi^{*} A\right) \forall g \in \mathcal{G}
$$

and therefore $A \rightarrow \Psi\left(\varphi^{*} A\right)$ is still a restricted gauge invariant function on $\mathcal{A}$.

Our "proof" of Eq. B.31) will boil down to formally verifying the hypothesis of Theorem B.14 in this infinite dimensional setting. It is worth noting that the results to follow hold for any $d \in \mathbb{N}$ with $d \geq 2$ in the special case where $\varphi(x)=R x+b$ with $R$ be a rotation on $\mathbb{R}^{d}$ and $b \in \mathbb{R}^{d}$. We now begin "verifying" the hypothesis of Theorem B.14 in this infinite dimensional gauge theory context.

Meta-Lemma B.49. The restricted gauge group, $\mathcal{G}$, is formally unimodular.

Meta-Proof. The Lie algebra $(\operatorname{Lie}(\mathcal{G}))$ of $\mathcal{G}$ consists of functions, $\xi: \mathbb{R}^{2} \rightarrow \mathfrak{k}$ with $\xi(0)=0$. Let

$$
\langle\xi, \eta\rangle_{\operatorname{Lie}(\mathcal{G})}:=\int_{\mathbb{R}^{2}}\langle\xi(x), \eta(x)\rangle_{\mathfrak{k}} d x
$$

where $\langle\cdot, \cdot\rangle_{\mathfrak{k}}$ is an $\operatorname{Ad}_{K}$ - invariant inner product on $\mathfrak{k}$. If $g \in \mathcal{G}$, then

$$
\left\|\operatorname{Ad}_{g} \xi\right\|_{\operatorname{Lie}(\mathcal{G})}^{2}=\int_{\mathbb{R}^{2}}\left|\operatorname{Ad}_{g(x)} \xi(x)\right|_{\mathfrak{k}}^{2} d x=\int_{\mathbb{R}^{2}}|\xi(x)|_{\mathfrak{k}}^{2} d x=\|\xi\|_{\operatorname{Lie}(\mathcal{G})}^{2}
$$

so that $\operatorname{Ad}_{g}$ acts as orthogonal transformation and therefore $\Delta_{\mathcal{G}}(g)=\left|\operatorname{det} \operatorname{Ad}_{g^{-1}}\right|=1$. Alternatively, extended $\langle\cdot, \cdot\rangle_{\operatorname{Lie}(\mathcal{G})}$ to a left invariant Riemannian metric on $T \mathcal{G}$ and note that the fact that $\operatorname{Ad}_{g}$ is an isometry for $\langle\cdot, \cdot\rangle_{\operatorname{Lie}(\mathcal{G})}$ implies this Riemannian metric is also right invariant. Thus the Riemannian volume measure associated to this Riemannian metric is both right and left invariant and so this measure is both a left and a right invariant Haar measure.

Lemma B.50. If $\varphi: \mathbb{R}^{2} \rightarrow \mathbb{R}^{2}$ is an area preserving diffeomorphism such that $\varphi(0)=0$, then $\gamma: \mathcal{G} \rightarrow \mathcal{G}$ defined by $\gamma(g)=\varphi^{*} g$ is a group isomorphism $!^{4}$ such that

$$
\varphi^{*}(A \cdot g)=\varphi^{*} A \cdot \gamma(g) \forall A \in \mathcal{A} \text { and } g \in \mathcal{G} \text {. }
$$

\footnotetext{
${ }^{4}$ The assumption $\varphi(0)=0$ is needed to guarantee that $\varphi^{*} g \in \mathcal{G}$ for every $g \in \mathcal{G}$.
} 
Proof. This result is a consequence of the following elementary computation;

$$
\begin{aligned}
\varphi^{*}(A \cdot g) & =\varphi^{*}\left(A^{g}\right)=\varphi^{*}\left[\operatorname{Ad}_{g^{-1}} A+g^{-1} d g\right] \\
& =\left[\operatorname{Ad}_{\left.(g \circ \varphi)^{-1} \varphi^{*} A+(g \circ \varphi)^{-1} d(g \circ \varphi)\right]}\right. \\
& =\varphi^{*} A \cdot \varphi^{*} g .
\end{aligned}
$$

Meta-Lemma B.51. Let $\varphi$ and $\gamma$ be as in Lemma B.50. Then $\gamma$ preserves Haar measure on $\mathcal{G}$ and hence $c_{\gamma}=1$ where $c_{\gamma}$ was defined in Eq. B.11).

Meta-Proof. For $g \in \mathcal{G}$ and $\xi \in \operatorname{Lie}(\mathcal{G})$ let $\tilde{\xi}(g)=L_{g *} \xi$ be the left invariant extension of $\xi$. Then

$$
\gamma_{*} \tilde{\xi}(g)=\left.\frac{d}{d t}\right|_{0} \gamma\left(g e^{t \xi}\right)=\left.\frac{d}{d t}\right|_{0} \gamma(g) \gamma\left(e^{t \xi}\right)=L_{\gamma(g) *} \gamma_{*} \xi=L_{\gamma(g) *}[\xi \circ \varphi] .
$$

By construction of the Riemannian metric on $\mathcal{G}, L_{\gamma(g) *}$ is an isometry and therefore (using $\varphi$ is area preserving) we find

$$
\begin{aligned}
\left\langle\gamma_{*} \tilde{\xi}(g), \gamma_{*} \tilde{\xi}(g)\right\rangle_{T_{\gamma(g)} \mathcal{G}} & =\langle\xi \circ \varphi, \xi \circ \varphi\rangle_{\operatorname{Lie}(\mathcal{G})} \\
& =\int_{\mathbb{R}^{2}}|\xi(\varphi(x))|_{\mathfrak{k}}^{2} d x=\int_{\mathbb{R}^{2}}|\xi(x)|_{\mathfrak{k}}^{2} d x=\langle\tilde{\xi}(g), \tilde{\xi}(g)\rangle_{T_{g} \mathcal{G}} .
\end{aligned}
$$

This shows $\gamma: \mathcal{G} \rightarrow \mathcal{G}$ is a Riemannian isometry and hence preserves the (fictitious) Riemannian volume measure on $\mathcal{G}$. As this volume measure is precisely Haar measure the "proof" is complete.

The last item we need to verify is that $\varphi^{*}: \mathcal{A} \rightarrow \mathcal{A}$ preserves Lebesgue measure when $\varphi$ is an area preserving diffeomorphisms on $\mathbb{R}^{2}$. To do this we will make use of the following meta-lemma.

Meta-Lemma B.52. Let $V$ be a finite dimensional inner product space and $U: \mathbb{R}^{2} \rightarrow$ End $(V)$ be a function such that $\operatorname{det} U(x)=1$ for all $x \in \mathbb{R}^{2}$. If $M_{U}: L^{2}\left(\mathbb{R}^{2} ; V\right) \rightarrow L^{2}\left(\mathbb{R}^{2} ; V\right)$ is the operation of multiplication by $U$, then $\operatorname{det} M_{U}=1$ or more usefully stated; the map, $f \rightarrow U f$, leaves Lebesgue measure invariant.

Meta-Proof. Here we suppose that $U(x)=U_{1}(x)$ where $\left\{U_{t}(x)\right\}_{t \in[0,1]}$ is a one parameter $\left(C^{1}\right.$ in $t$ ) family of functions in $S L(V)$ with $U_{0}(x)=I$ for all $x$. Further let $\alpha_{t}(x):=U_{t}(x)^{-1} \dot{U}_{t}(x)$ so that $\dot{U}_{t}(x)=\alpha_{t}(x) U_{t}(x)$ with $U_{0}(x)=I$ and $\operatorname{tr}\left(\alpha_{t}(x)\right)=0$. We then formally should have,

$$
\frac{d}{d t} \operatorname{det}\left[M_{U_{t}}\right]=\operatorname{det}\left[M_{U_{t}}\right] \operatorname{Tr}\left[M_{U_{t}^{-1}} M_{\dot{U}_{t}}\right]=\operatorname{det}\left[M_{U_{t}}\right] \operatorname{Tr}\left[M_{\alpha_{t}}\right]
$$

where $\operatorname{Tr}$ is the infinite dimensional trace on $L^{2}\left(\mathbb{R}^{d}, V\right)$. To evaluate the trace, let $\left\{u_{m}\right\}_{m=1}^{\infty}$ be an orthonormal basis for $L^{2}\left(\mathbb{R}^{d}, \mathbb{R}\right)$ and $\left\{e_{i}\right\}_{i=1}^{\operatorname{dim} V}$ be an orthonormal basis for $V$ relative to some fixed inner product on $V$. Then $\left\{u_{m} \cdot e_{i}: m \in \mathbb{N} \& 1 \leq i \leq \operatorname{dim} V\right\}$ is an orthonormal basis for 
$L^{2}\left(\mathbb{R}^{d}, V\right)$ and hence it is reasonable to compute $\operatorname{Tr}\left(M_{\alpha_{t}}\right)$ as,

$$
\begin{aligned}
\operatorname{Tr}\left(M_{\alpha_{t}}\right) & =\sum_{m=1}^{\infty} \sum_{i=1}^{d}\left\langle M_{\alpha_{t}} u_{m} \cdot e_{i}, u_{m} \cdot e_{i}\right\rangle_{L^{2}\left(\mathbb{R}^{d}, V\right)} \\
& =\sum_{m=1}^{\infty} \sum_{i=1}^{d} \int_{\mathbb{R}^{2}}\left\langle\alpha_{t}(x) e_{i}, e_{i}\right\rangle u_{m}^{2}(x) d x \\
& =\sum_{m=1}^{\infty} \int_{\mathbb{R}^{2}} \operatorname{tr}\left[\alpha_{t}(x)\right] u_{m}^{2}(x) d x=\sum_{m=1}^{\infty} \int_{\mathbb{R}^{2}} 0 \cdot u_{m}^{2}(x) d x=0 .
\end{aligned}
$$

Thus we have shown $\operatorname{det}\left[M_{U_{t}}\right]$ is constant in $t$ and so

$$
\operatorname{det} M_{U}=\operatorname{det}\left[M_{U_{1}}\right]=\operatorname{det}\left[M_{U_{0}}\right]=\operatorname{det} I_{L^{2}\left(\mathbb{R}^{2} ; V\right)}=1 .
$$

Remark B.53. The computation of the trace of $M_{\alpha_{t}}$ above is certainly not rigorous as $M_{\alpha_{t}}$ is not a trace class operator.

Meta-Proposition B.54. If $\varphi: \mathbb{R}^{2} \rightarrow \mathbb{R}^{2}$ is an area preserving diffeomorphism, then the induced map, $\mathcal{A} \ni A \rightarrow \varphi^{*} A \in \mathcal{A}$ formally preserves Lebesgue measure on $\mathcal{A}$.

Meta-Proof. If $A=A_{1} d x_{1}+A_{2} d x_{2}$, then

$$
\begin{aligned}
\varphi^{*} A & =A_{1} \circ \varphi d\left[x_{1} \circ \varphi\right]+A_{2} \circ \varphi d\left[x_{2} \circ \varphi\right] \\
& =A_{1} \circ \varphi\left[\partial_{1} \varphi_{1} d x_{1}+\partial_{2} \varphi_{1} d x_{2}\right]+A_{2} \circ \varphi\left[\partial_{1} \varphi_{2} d x_{1}+\partial_{2} \varphi_{2} d x_{2}\right] \\
& =\left(\left[A_{1} \circ \varphi\right] \partial_{1} \varphi_{1}+\left[A_{2} \circ \varphi\right] \partial_{1} \varphi_{2}\right) d x_{1}+\left(\left[A_{1} \circ \varphi\right] \partial_{2} \varphi_{1}+\left[A_{2} \circ \varphi\right] \partial_{2} \varphi_{2}\right) d x_{2} .
\end{aligned}
$$

Thus identifying $A$ with $\left[\begin{array}{ll}A_{1} & A_{2}\end{array}\right]^{\text {tr }}$, the transformation $\mathcal{A} \ni A \rightarrow \varphi^{*} A \in \mathcal{A}$ is the composition of the linear transformation

$$
\left[\begin{array}{l}
A_{1} \\
A_{2}
\end{array}\right] \rightarrow\left[\begin{array}{l}
A_{1} \\
A_{2}
\end{array}\right] \circ \varphi
$$

followed by applying the linear transformation, $M_{U}$, where

$$
U(x, y):=\left[\begin{array}{ll}
\left(\partial_{1} \varphi_{1}\right)(x, y) & \left(\partial_{1} \varphi_{2}\right)((x, y)) \\
\left(\partial_{2} \varphi_{1}\right)(x, y) & \left(\partial_{2} \varphi_{2}\right)((x, y))
\end{array}\right] .
$$

The assumption that $\varphi$ is area preserving is equivalent to $\operatorname{det} U(x, y)=1$ and hence by MetaLemma B.52, Det $\left[M_{U}\right]=1$. The assumption that $\varphi$ is area preserving also implies that transformation in Eq. B.33 is an isometry on $L^{2}\left(\mathbb{R}^{2} ; \mathfrak{k}^{2}\right)$ and so again (formally) preserves Lebesgue measure. As $A \rightarrow \varphi^{*} A$ is a composition of two Lebesgue measure preserving maps it also (formally) preserves Lebesgue measure on $\mathcal{A}$.

Meta-Theorem B.55. Let $d=2$. If $\varphi$ is an area preserving diffeomorphism such that $\varphi(0)=0$ and $\Psi$ is a restricted gauge invariant function, then formally Eq. (B.31) holds.

Proof. This result heuristically follows from Theorem B.14 whose hypothesis have been heuristically verified in Meta-Lemmas B.49 and B.51 and Meta-Proposition B.54.

Meta-Theorem B.56. Let $d=2$. If $\varphi$ is an area preserving diffeomorphism and $\Psi$ is invariant under the full gauge group, then (formally) Eq. B.31) still holds. 
Proof. If $\varphi(0)=0$ the result follows from Meta-Theorem B.55. When $\varphi(0) \neq 0$ we let $\eta(\cdot):=\varphi(\cdot)-\varphi(0)$. Then $\varphi=\eta+\varphi(0)$ which shows $\varphi$ is the composition of an area preserving diffeomorphism $(\eta)$ fixing $0 \in \mathbb{R}^{2}$ followed by translation by $\varphi(0)$. Thus to finish the proof it suffices to consider the special case where $\varphi(x)=x+b$ for some vector $b \in \mathbb{R}^{2}$. We can further reduce the problem to the case where $b \in \mathbb{R} e_{1}$ where $e_{1}=(0,1)$. To verify this, let $R$ be the $2 \times 2$ rotation matrix such that $R^{-1} b=v \in \mathbb{R} e_{1}$ and then write $\varphi$ as $\varphi(x)=R\left[R^{-1} x+v\right]$. This shows that $\varphi$ is a composition of two area preserving diffeomorphism, $R$ and $R^{-1}$, which fix $0 \in \mathbb{R}^{2}$ along with a translation by $v \in \mathbb{R} e_{1}$.

Owing to the above reductions we now assume that $\varphi(x)=x+\lambda e_{1}$ for some $\lambda \in \mathbb{R}$. Let $\sigma$ be the complete axial homotopy in Example B.20 in which case $A \in \mathcal{A}_{\sigma}$ iff $A=A_{1} d x_{1} \in \mathcal{A}_{\sigma}$ with $A_{1}\left(x_{1}, 0\right)=0$ for all $x_{1} \in \mathbb{R}$. Since, for $A \in \mathcal{A}_{\sigma}$

$$
\varphi^{*}\left(A_{1} d x_{1}\right)=A_{1} \circ \varphi d x_{1}=A_{1}(\cdot+\lambda, \cdot) d x_{1}
$$

it follows that $\varphi^{*}$ preserves $\mathcal{A}_{\sigma}$. Moreover, as $\varphi^{*}$ acts orthogonally on $\mathcal{A}_{\sigma}$ equipped with the $L^{2}\left(\mathbb{R}^{2}, \mathfrak{k}\right)$-inner product it is reasonable to (formally) assert that $\varphi^{*}$ leave "Lebesgue measure" on $\mathcal{A}_{\sigma}$ invariant. As $m_{\sigma}$ is formally a Lebesgue measure on $\mathcal{A}_{\sigma}$ by Meta-Proposition B.40, we conclude that

$$
\begin{aligned}
f_{\mathcal{A}} \Psi\left(\varphi^{*} A\right) d m(A) & =\int_{\mathcal{A}_{\sigma}} \Psi\left(\varphi^{*} A\right) d m_{\sigma}(A) \\
& =\int_{\mathcal{A}_{\sigma}} \Psi(A) d m_{\sigma}(A)=f_{\mathcal{A}} \Psi(A) d m(A) .
\end{aligned}
$$

\section{REFERENCES}

[1] Guillaume Cébron, Antoine Dahlqvist, and Franck Gabriel, The generalized master fields, Journal of Geometry and Physics 119 (2017), 34-53. MR 3661522

[2] Nelia Charalambous and Leonard Gross, The Yang-Mills heat semigroup on three-manifolds with boundary, Comm. Math. Phys. 317 (2013), no. 3, 727-785. MR 3009723

[3] _ Neumann domination for the Yang-Mills heat equation, J. Math. Phys. 56 (2015), no. 7, $073505,21$. MR 3405967

[4] Initial behavior of solutions to the Yang-Mills heat equation, J. Math. Anal. Appl. 451 (2017), no. 2, 873-905. MR 3624771

[5] S. Chatterjee, Rigorous solution of strongly coupled $S O(N)$ lattice gauge theory in the large $N$ limit, ArXiv e-prints (2015).

[6] S. Chatterjee and J. Jafarov, The $1 / N$ expansion for $S O(N)$ lattice gauge theory at strong coupling, ArXiv e-prints (2016).

[7] Sourav Chatterjee, The leading term of the Yang-Mills free energy, J. Funct. Anal. 271 (2016), no. 10, 29443005. MR 3548284

[8] A. Dahlqvist and J. Norris, Yang-Mills measure and the master field on the sphere, ArXiv e-prints (2017).

[9] Antoine Dahlqvist, Free energies and fluctuations for the unitary Brownian motion, Comm. Math. Phys. 348 (2016), no. 2, 395-444. MR 3554890

[10] Claude Dellacherie, Capacités et processus stochastiques, Springer-Verlag, Berlin, 1972, Ergebnisse der Mathematik und ihrer Grenzgebiete, Band 67. MR MR0448504 (56 \#6810)

[11] Eric DHoker, Quantum field theory: Part I, Department of Physics and Astronomy, UCLA, Lecture Notes, October 2004. http://www.pa.ucla.edu/content/eric-dhoker-graduate-lecture-notes

[12] Bruce K. Driver, Classifications of bundle connection pairs by parallel translation and lassos, J. Funct. Anal. 83 (1989), no. 1, 185-231. 
[13],$Y M_{2}$ : continuum expectations, lattice convergence, and lassos, Comm. Math. Phys. 123 (1989), no. 4, $575-616$.

[14] Bruce K. Driver, Franck Gabriel, Brian C. Hall, and Todd Kemp, The Makeenko-Migdal equation for YangMills theory on compact surfaces, Comm. Math. Phys. 352 (2017), no. 3, 967-978. MR 3631396

[15] Bruce K. Driver, Brian C. Hall, and Todd Kemp, Three proofs of the Makeenko-Migdal equation for Yang-Mills theory on the plane, Comm. Math. Phys. 351 (2017), no. 2, 741-774. MR 3613519

[16] Rajesh Gopakumar and David J. Gross, Mastering the master field, Nuclear Phys. B 451 (1995), no. 1-2, 379-415. MR 1352420

[17] L. Gross, The Yang-Mills heat equation with finite action, ArXiv e-prints (2016).

[18] Leonard Gross, A Poincaré lemma for connection forms, J. Funct. Anal. 63 (1985), no. 1, 1-46. MR 795515

[19] Leonard Gross, Christopher King, and Ambar Sengupta, Two-dimensional Yang-Mills theory via stochastic differential equations, Ann. Physics 194 (1989), no. 1, 65-112.

[20] B. C. Hall, The large-N limit for two-dimensional Yang-Mills theory, ArXiv e-prints (2017).

[21] Svante Janson, Gaussian Hilbert spaces, Cambridge Tracts in Mathematics, vol. 129, Cambridge University Press, Cambridge, 1997. MR MR1474726 (99f:60082)

[22] V. A. Kazakov and I. K. Kostov, Nonlinear strings in two-dimensional U( $\infty)$ gauge theory, Nuclear Phys. B 176 (1980), no. 1, 199-215. MR 596907 (81k:81101)

[23] V.A. Kazakov, Wilson loop average for an arbitrary contour in two-dimensional u(n) gauge theory, Nuclear Physics B 179 (1981), no. 2, 283 - 292.

[24] Thierry Lévy, The master field on the plane, Astérisque (2017), no. 388, ix+201. MR 3636410

[25] Yu. M. Makeenko and A. A. Migdal, Exact equation for the loop average in multicolor QCD, Physics Letters B 88 (1979), no. 1, 135 - 137.

[26] T. Nguyen, Quantum Yang-Mills Theory in Two Dimensions: Exact versus Perturbative, ArXiv e-prints (2015).

[27] _ Stochastic Feynman Rules for Yang-Mills Theory on the Plane, ArXiv e-prints (2016).

[28] _ Wilson Loop Area Law for 2D Yang-Mills in Generalized Axial Gauge, ArXiv e-prints (2016).

[29] Timothy Nguyen, The perturbative approach to path integrals: a succinct mathematical treatment, J. Math. Phys. 57 (2016), no. 9, 092301, 27, See Section III for gauge fixing from a differential form point of view. MR 3550290

[30] Ambar Sengupta, Quantum Yang-Mills theory on compact surfaces, Stochastic analysis and applications in physics (Funchal, 1993), NATO Adv. Sci. Inst. Ser. C Math. Phys. Sci., vol. 449, Kluwer Acad. Publ., Dordrecht, 1994, pp. 389-403. MR 1337973

[31] _ Gauge theory on compact surfaces, Mem. Amer. Math. Soc. 126 (1997), no. 600, viii+85. MR 1346931

[32] _ Yang-Mills on surfaces with boundary: quantum theory and symplectic limit, Comm. Math. Phys. 183 (1997), no. 3, 661-705. MR 1462231

[33] Ambar N. Sengupta, Traces in two-dimensional QCD: the large-N limit, Traces in number theory, geometry and quantum fields, Aspects Math., E38, Friedr. Vieweg, Wiesbaden, 2008, pp. 193-212. MR 2494192 (2010g:81165)

[34] Steven Weinberg, The quantum theory of fields. Vol. II, Cambridge University Press, Cambridge, 2005, Modern applications. MR 2148467

Department of Mathematics, University of California, San Diego, La Jolla, CA 92093-0112, BDRIVERQMATH . UCSD. EDU 